
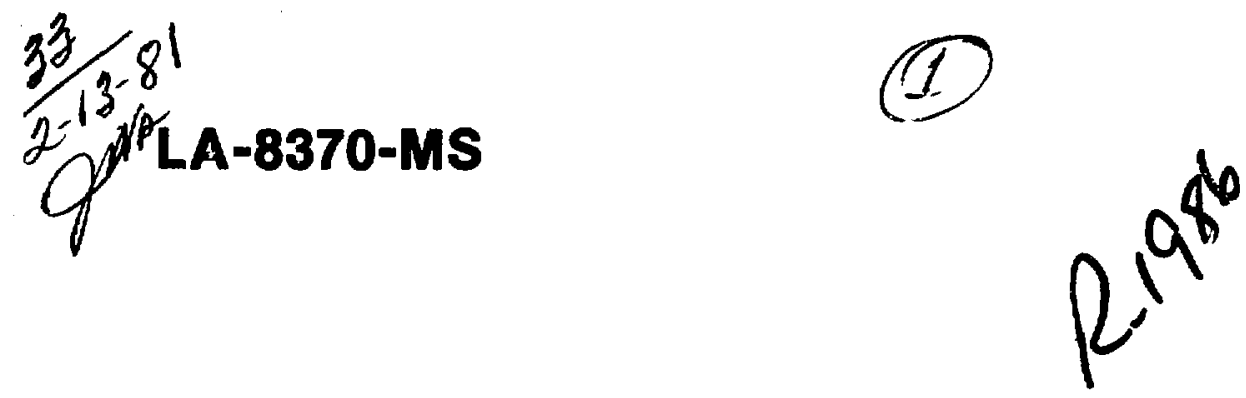

The Dynamic Theory: A New View of Space, Time, and Matter

莫

¿

竞

\$)

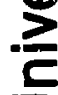

5

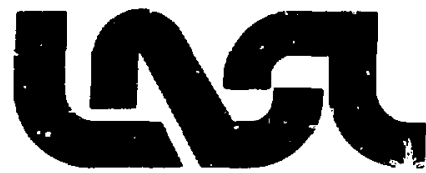




\title{
LA-8370-MS
}

UC-34

Issued: December 1980

\section{The Dynamic Theory: \\ A New View of Space, Time, and Matter}

\author{
Pharis E. Williams
}

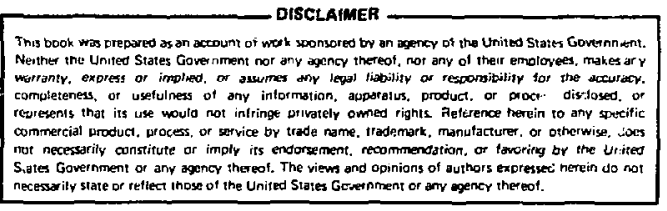


CONTENTS

I. INTRODUCTION

II. UNIFYING EFFECT OF THE DYNAMIC THEORY 7

A. General Laws 8

1. First Law 8

2. Second Law 11

3. The Absolute Velocity 18

4. The Concept of Entropy 20

5. The Third Law 23

B. Genera7 Maxwe11 and Energy Relations 25

C. Equilibrium and Stability Conditions 28

1. Equilibrium Conditions 28

2. Stability Conditions $\quad 30$

D. Geometry and Field Equations 33

1. Geometry 34

2. Field Equations 50

E. Mechanical Systems Near Equilibrium 51

1. Classical Mechanics 52

2. Relativistic Mechanics 54

3. Non-Isolated Systems 64

F. Quantum Effects $\quad 64$

G. Summary $\quad 74$

III. FIVE-DIMENSIONAL SYSTEMS 76

A. Systems Near an Equilibrium State 78

1. Equations of Motion 78

2. Energy Equation 83 
B. Systems with Non-Euclidean Manifold 85

1. General Variational Principle 85

2. Gauge Function Field Equations 87

3. Interpretation of the Current Densities 91

4. Additional Developments of the Five-Dimensional 92

Field

a. Energy-Momentum Tensor 92

b. Force Density Vector 96

c. Equation of Energy Flow 98

d. Momentum Conservation 99

e. Eauge Fie?d Pressure 100

f. Self Energy of a Charged Particie 103

C. Quantization in Five Dimensions 111

1. Quantization 111

2. Five-Dimensional Hamiltonian 112

3. Five-Dimensionai Dirac Equation 113

4. "Lorentz" Covariance 115

5. Spin 116

6. Dirac Equation with Fields 117

7. Allowed Fundamental Spin States 119

D. Mass Conservation Hydrodynamic Systems 124

1. Surface Geometry 125

a. First Fundamenta1 Quadratic Form 128

b. Second Fundamental Quadratic Form 132

c. Tensor Derivatives 136

2. Relativistic Hydrodynamics 145

3. Classica1 Hydrodynamics 146

$\begin{array}{ll}\text { 4. Shock Waves } & 149\end{array}$

E. Mass Conservative Electrodynamics 150 
F. Field Equations $\quad 156$

1. Nonlinear Field Equations 157

2. Linearization of Fieíld Equations 161

IV. CONCLUSIONS 162 
THE DYNAMIC THEORY: A NEW VIEW OF SPACE, TIME, AND MATTER

\author{
by \\ Pharis E. Williams
}

\begin{abstract}
The theory presented represents a different approach toward unification of the various branches of physics. The foundation of the theory rests upon generalizations of the classical laws of thermodynamics, particularly Caratheodory's abstract statement of the second law. These adopted laws are shown to produce, as special cases, current theories such as: Einste in's General and Special Relativity, Maxwell's electromagnetism, classical thermodynamics, and quantum principles.

In addition to this unification, the theory provides predictions that may be experimentally investigated. Some of the predictions are: a limiting rate of mass conversion, reduced pressures in electromagnetically contained plasmas, increased viscous effects in shocked materials, a finite self energy for a charged particle, and the possible creation of particles with velocities greater than the speed of light.
\end{abstract}

\title{
I. INTROOUCTION
}

The objective of this report is to present as complete a picture as possible of the development and current status of the proposed physical theory hereafter referred to as the Dynamic Theory. The presentation will include philosophical foundations, logic development, and some of the unanswered questions considered. Such a presentation runs the risk of being lengthy and appearing to lack logical order, particularly if strict chronological order is used. This risk will be taken in order to make the presentation as complete as possible though chronological order will not be followed. 
During any theorization the philosophy of the theorist plays such an important role that an attempt to understand the theory is aided by a knowledge of this philosophy. Therefore this report includes not only the philosophical basis upon which the theory is based and the mathematical development but also ideas and beliefs which played a part in the various decisions. Because of the individualistic nature of philosophy this report will deviate occasionally from a strict third person presentation, risking a loss of professional appearance, to the clearly personal first person.

Though I had often asked "Why?" when confronted with some new assumption or adopted postulate, the first really puzzling facet of current physics I encountered was the concept of relativistic kinetic energy from Einstein's Special Theory of Relativity. The puzzling part was that it depended upon the speed of light independent of the mechanism by which this energy might be transferred. To better illustrate what puzzled me, consider the transfer of energy between two charged particles on collision courses. If the particles have near-miss trajectories, then the energy is primarily transferred by the electrical forces between the charges. From the view of retarded potentials, or the concept of a limiting speed of electromagnetic signal transmission, it is rather easy to accept the energy transferred being dependent upon this limiting velocity. But suppose the particles are uncharged and che interaction is strictly a gravitational one. Again the concept of a limiting signal speed would imply that the energy exchanged between the particles depend upon this limiting velocity. But is it the same as the limiting signal velocity for the electromagnetic case? Do gravitational waves travel at the same speed as electromagnetic waves?

Einstein, in the Special Theory of Relativity, adopted the position that the constancy of the speed of light forces a modification of Newton's dynamic law. This modification implies that all forces have the same limiting velocity, namely, the speed of light. There exists an abundance of theoretical and experimental evidence that the speed of light becomes the limiting velocity whenever electromagnetic forces are involved. The point that bothered me was whether other forces, such as gravitational, should also have the same limiting velocity. Though we have had reports of the detection of gravitational waves, we have no experimental determination of the speed of a gravitational wave. Therefore, I object to the viewpoint that the modification to Newton's law should be applied to all forces without some additional justification. 
Let me describe an analogy which may not hold in the strictest sense yet will serve to illustrate my point of view. A river, flowing toward the sea, carries energy with it. The speed with which this energy can move from one point to another is the velocity of the river's current. The river produces a force on a boat tied up to a pier on the river. When the boat is set adrift, this force accelerates the boat. However, the maximum velocity to which the river can accelerate the boat is the current velocity; this is the velocity with which the energy of the river can propagate.

From this point of view the speed of light, being the propagation velocity of electromagnetic energy must be the limiting velocity associated with electromagnetic forces. Certainly nature would be much simpler if all forces have the same limiting velocity. Yet without some experimental evidence of the propagation of gravitational energy, I find it difficult to feel comfortable with Einstein's modification of Newton's law justified by electromagnetic experimental evidence and arguments of simplicity.

The fundamental philosophical viewpoint that the force depends upon velocity and vanishes as the velocity approaches the limiting velocity raises another question concerning Einstein's modification of classical mechanics. Under Einstein's modification Hamilton's principle is written with a relativistic mass which depends upon the velocity and a velocity independent force. Does this represent a different philosophy or are both views equivalent? More specifically, are the "real" concepts to be taken as a mass independent of velocity together with a velocity dependent force or should we associate the velocity dependent relativistic mass and velocity independent forces with "real" world? Or does it make any difference which we chose?

At this point I faced the first major decision. If I adopted Einstein's postulates, then it appeared that I would be required to change my intuitive belicfs concerning certain physical phenomena. I found this extremely difficult to do. On the other hand, if I did not embrace these posculates, I would have to replace them with something that would say essentially the same thing in all cases where the Special Theory of Relativity has been found to be very accurate. Not only this but if a new point of view were adopted, then virtually the entire sphere of physics may need to be reviewed in order to ensure that the new point of view did not conflict with currently used theories. This seemed an imposing, if not impossible task, particularly since my educational experience was in eiectrical engineering rather than physics. 
History records the advancements in physics which came from the efforts of people new to the fieid. Therefore my lack of training in physics might be turned inio an advantage if I sought to determine a philosophical basis unhampered by the directed philosophy that comes from a study of physics as currentiy taught. This is in contradistinction with current practices and procedures of academicism where mastery of current theories generally precedes the development of a new one. To deliberately choose this deviation risks accusations of arrogance and naivete. On the other hand such a choice seemed the best way of avoiding the danger of becoming so familiar with current ways of thinking as to make it imp, obable of giving due attention to other ways. Whether or not $I$ succeeded in determining an alternate set of postulates consistent with reality, the search would demand a deeper study of physics than I would likely achieve otherwise.

Having decided to look for a new foundation for physics I was faced with the question of how to begin. I recalled some Ozark hill philosophy I overheard as a youngster. A native Ozarkian was giving directions to a stranger who was trying to find a certain fishing hole. The directions went something like this: "See yonder road going dowr that holler? Weil, go down thar 'bout five mile and you'll come to a fork in the road. Take the right hand fork. Now that's the wrong one but you take it anyways. After you've gona a piece, you' 11 come to a $\log$ across the road. Now you know you're on the wrong road. So go back and take the left hand fork. You can't miss it."

A quick review of physics reveals that there are different branches with different sets of fundamental laws or postulates. Though it is easy to see how the distinction between these branches came about, it was difficult for me to believe that nature shared the same divisions. I felt that all natural phenomena should be explained by a single set of fundamental laws. This belief is somewhat like a grove of redwood trees or bamboo forest. Above the ground each tree appears as a distinct plant. Yet we know that below the ground they may be found to grow from the same root system. Thus i felt that a more fundamental approach might display the unity in nature and that prior attempts at unification in the search for a unified field theory could be likened to attempts to tie the trees together at the tree top level rather than down at the root level.

Is nature symmetrical in time? Does everything run backward in time as well as forward? Obviously, not every process in nature will run backwards, yet the equations of motion in Newtonian and relativistic mechanics are time symmetrical. 
I believe in an asymmetrical nature and this belief played a role in the eventual selection of fundainental laws.

How then did I use this philosophy to determine a set of generalized laws on which to base an attempt to construct a new approach to physics?

Newtonian mechanics fails to describe events involving high velocities, relativistic mechanics fails to describe the atom, and gravitational effects have resisted quantization. If these are viewed as logs and the 0zarkian's directions are followed, then we must retrace our steps and seek another approach rather than attempting to chop up the $\log$ and continue to push forward up one of these roads.

The branch of thermodynamics, however, does not appear to have a $\log$ somewhere along the way. Here the classical tiermodynamic laws are very general, particularly Caratheodory's statement of the second law. Thus the thermodynamic laws appeared to be the fork in the road where a new route might be chosen.

However, in mechanics we talk of equations of motion, field equations, and geometry while in thermodynamics we speak of equations of state and equilibrium. If a generalization of the classical thermodynamic laws is adopted, how might we obtain the equations with which we are familiar in mechanics? More particularly, how could this type of general law yield geometry and a variational principle? The second law of thermodynamics can produce a variational principle through principles such as increasing entropy and minimizing free energy, but can it also produce a geûietry?

This seemed to be a crucial point. If the laws could not produce a geometry, then a geometry would have to be assumed, thus necessitating an additional assumption. The belief that a simple fundamental set of laws should lead to the fundamental principles of the different branches of physics made the thought of additional assumptions abhorrent. The notion that the adopted laws should specify the type of geometry that must be used seemed very satisfying. Newton found that the absolute nature of Euclidean geometry brought undesirable features. Einstein, in his General Theory, displayed the benefits that might be gained by going to a more general geometry. He showed that physical phenomena might be displayed as elements determined by certain physical laws. This is essentially the question here. Can a set of laws, which are generalizations of the classical thermodynamic laws, determine the metric elements and hence the geometry? 
By appealing to the mathematics of functions of more than one variable we find that a quadratic form becomes involved when a maximum or minimum is sought. Further, this quadratic form generates a natural geometry for that function. In thermodynamics the stability conditions provide a similar quadratic form and therefore the quadratic form which specifin: the stability conditions should form a natural geometry for a physical system governed by laws such as the thermodynamic laws.

Thus the foundations of the theory have been outlined, namely the belief that all physical phenomena should be derivable from a single set of physical laws which are generalizations of the classical thermodynamic laws. Such a theory should be capable of describing all the dynamic events in nature. Therefore it seems appropriate to call it the "Dynamic Theory." Obviously, for such a theory to be tenable it must reproduce, or be consistent with, the various fundamental postulates and/or laws currently used in the various branches of physics. Indeed it should do even more. It should also reduce the number of necessary assumptions and provide an unprecedented unification of physics. Further, there is the possibility that the theory might produce an experimentally verifiable prediction.

The first requirement that should be placed upon the Dynamic Theory is that it reproduce, or be consistent with, current theories. In order to show that the Dynamic Theory satisfies this requirement, Section II of this report states the adopted laws and then shows how appropriate restrictions upon the system do yield the fundamental principles for the various theories.

Though a theory which has the capability of displaying a unification of physical theories might have significant value based solely upon this capability, it would become more attractive if it could explain phenomena for which no explanation exists or make some new prediction which might lead to an experimen.. tal test of the theory. Since restrictions were placed upon the system in order to show how current theories may be obtained, the easiest way to see the expanded coverage of the theory is to relax one or more of the restrictions and consider a more general system. In Section III one of the previously imposed restrictions is relaxed and the results are worked out for several types of systems.

A theory, such as the Dynamic Theory, immediately poses several problems which are not associated with validity or applicability. First, there is a new point of view to be dealt with. Initially it would appear to be inconsistent 
with all past concepts of system energy or relativistic concepts. Yet in the end it is completely consistent with current theories and sheds an entirely new light upon physical phenomena.

Another imposing difficulty with the Dynamic Theory stems from its generality. The scope of the theory includes all physical phenomena while in the past half century the vast amount of scientific knowledge that has been accumulated has demanded specialists. Increasing expansion of mankind's knowledge demands further specialization. Such a progression produces no demand for a generalist. The result is that the greater portion of this theory will be outside the field of many readers.

Closely associated with this problem is another. Throughout science symbols and words are used to denote concepts and quantities. The limited number of available symbols and words together with the expanded scope of scientific knowledge requires duplication. For the specialists this duplication can be somewhat minimized. However, in the case of a general theory touching virtually all areas of specialization the problem becomes very significant. In particular, if a certain symbol or set of words is used, a certain notion or concept may be associated with them by the reader. This association will likely depend upon the reader's specialty and therefore will vary with the reader. Any attempt to choose symbology or word usage aimed at a particular speciality risks increased confusion for readers in other fields. Therefore the reader is cautioned to keep in mind that conceptualizations and symbology familiar because of its use in one branch of physics may now take on an entirely rew meaning.

\section{UNIFYING EFFECT OF THE DYNAMIC THEORY}

The Dynamic Theory uses a different viewpoint, or approach, to present a description of physical phenomena. Therefore the first criterion that it must meet is that it must not be in conflict with existing theories in a field of physics where the existing theory gives an adequate and accurate description. To show that the Dynamic Theory meets this criterion, this section will present the adopted laws and then proceed to show how the fundamental principles of existing theories may be obtained from these laws. This is in essence displaying the unifying effect of the theory. 


\section{A. General Laws}

In the following development physical concepts are necessary, as are symbols for these concepts. Because this development will merge certain thermodynamic conceptualizations into mechanics, a notational dilemma must be faced. On the one hand, it is desired to preserve the thermodynamic conceptualization by using familiar symbols from that theory. On the other hand, descriptions of mechanical systems are also sought. The formulism then looks either like thermodynamics with familiar thermodynamic quantities replaced by mechanical quantities, or it looks like mechanics into which thermodynamic quantities intruded. In either case there is danger of confusion. One could avoid the dilemma by choosing entirely different symbols for the variables of the theory. But then the whole takes an artificially abstract character. Since the purpose of this formulation is to bring out the power of the thermodynamic conceptualization, it was decided to use the suggestiveness of the thermodynamic or mechanical symbols whenever convenient; the reader is asked to keep an open mind and not make premature associations with the symbols used.

1. First Law. The concept of conservation of energy is fundamental to all branches of physics and therefore represents a logical beginning for a generalized theory. Therefore, in terms of generalized coordinates or independent variables, the notion of work, or mechanical energy, is considered linear forms of the type

$$
\bar{d} W=F_{i}\left(q^{1}, \ldots, q^{n}, \dot{q}^{1}, \ldots, \dot{q}^{n}\right) d g^{i} \quad(i=1,2, \ldots, n),
$$

where the forces $F_{i}$ may be functions of the velocities $\left(\mathrm{dq}^{i} / \mathrm{dt}=\dot{q}^{i}\right)$ as well as the coordinates $q^{i}$ and the summation convention is used. The inclusion of velocities in forces reflects the belief that forces should depend upon the velocities. This will become clearer when these work terms are included in the first law.

The line integral $\int_{C} F_{j} d q^{i}$ then represents the work done along the path $C$ by the generalized forces.

A system may acquire energy by other means in addition to the work terms; such energy acquisition is denoted $\bar{d} E$.

The system energy, which represents the enerny possessed by the system, is considered to be 


$$
u\left(q^{1}, \ldots, q^{n}, \dot{q}^{1}, \ldots, \dot{q}^{n}\right)
$$

$\mathrm{d} U$ will be assumed to be a perfect differential.

With these concepts, then the generalized law of conservation of energy, which is adopted as the first law of the Dynamic Theory, has the form

$$
\begin{aligned}
\overline{\mathrm{d}} E & \equiv \mathrm{d} U-\overline{\mathrm{d}} W \\
& =\mathrm{d} U-F_{i} \mathrm{dq}^{i} \quad(i=1, \ldots, \mathrm{n}) .
\end{aligned}
$$

Positive $\overline{\mathrm{d}} E$ is taken as energy added to the system by means other than through the work terins and $F_{i}$ is taken as the component of the generalized force acting on the system which caused a displacement $\mathrm{dq}^{i}$.

In the first law the dimensionality is $n+1$ and is determined by the system considered. There is no limitation on the quantity or type of variables that may be used. However, in this presentation and in practice, it will be beneficial to place restrictions upon the type and number of allowed work terms. A system with only one work term which is the $p d v$ expansion work of classical thermodynamics will be called a "thermodynamic" system and the dimensionality will be two. A system with three or less mechanical $f d x$ work terms will be called a "mechanical" system with the appropriate dimensionality. Obviously, if there are three mechanical work terms, the dimensionality will be four. A system with a combination of thermodynamic and mechanical work terms will be considered later.

In an infinitesimal transformation, the first law is equivalent to the statement that the differential

$$
\mathrm{d} U=\overline{\mathrm{d}} E+F_{\mathbf{j}} \mathrm{dq}{ }^{\mathbf{i}}
$$

is exact. That is, there exists a function $U$ whose differential is $\mathrm{d} U$; or the integral $\int \mathrm{d} U$ is independent of the path of the integration and depends only on the limits of integration. This condition is not shared by $\overline{\mathrm{d}} E$ or $\overline{\mathrm{d}} W$. The path dependence of $\int \bar{d} W$ is another reason that the generalized forces are assumed to be functions of velocity as well as position. In Newtonian mechanics forces are usually assumied to be dependent on position only so that the simplicity of path independence may be used. Thuugh even in Newtonian mechanics certa in forces are 
taken as velocity dependent. Friction forces are an example.

This statement of the generalized first law is consistent with the first law of thermodynamics in that if there is only one generalized force, which is taken to be the pressure, and one generalized coordinate, the volume, then Eq. (1) comes

$$
\overline{d Q} \equiv \bar{d}_{E}=\mathrm{d} U+P d v
$$

where $F=-P$ with the convention that work of exparision is work done by the sys$+ \pm m$ on its surroundings. Here the system energy, $U$, is the thermodynamical internal energy. There should then be no confusion when Caratheodory's statement of the second law is applied to this thermodynamic system. However, when considering the application of generalizations of ihe classical thermodynamic laws to mechanical systems some confusion may be expected. During the initial portion of this development, it is desired to demonstrate the applicability of the genfralized laws to mechanical systems. Therefore, it may help to avoid confusion to think of the generalized coordinates of a mechanical system as the space coordinates of a mass point. Obviously, there exist systems in nature that may be considered to consist of a continuous distribution of mass points. Such a system may be thought of as a composite system of an infinite number or subsystems and therefore involve an infinite number of "generalized coordinates," or "degrees of freedom." However, just as in classical mechanics, we may later make the transition from mass points to matter in bulk; then the generalized coordinates, $q^{i}$, used here may better be termed independent variables.

To explore some of the consequences of the exactness of $d U$, consider a system whose variables are $F, \dot{q}$ and $q$. The existence of the state function $U$, or an equation of state, means that any pair of these three parameters may be chosen to be the independent variables that completely specify the system. For example consider $U=U(F, q)$, then

$$
\mathrm{d} U=\left(\frac{\partial U}{\partial F}\right)_{q} \mathrm{~d} F+\left(\frac{\partial U}{\partial q}\right)_{F} \mathrm{dq}
$$

The requirement that $\mathrm{d} U$ be exact immediately leads to the result

$$
\frac{\partial}{\partial q}\left[\left(\frac{\partial U}{\partial F}\right)_{q F}=\frac{\partial}{\partial F}\left[\left(\frac{\partial U}{\partial q}\right)_{F}\right]\right. \text {. }
$$


The "energy capacity" of a system at the position $q$ with $d q=0$ may be defined as

$$
c_{q} \equiv\left(\frac{\Delta E}{\Delta \tilde{q}_{q}}\right)_{q}=\left(\frac{\partial U}{\partial \dot{q}}\right)_{q},
$$

and the "energy capacity" of a system under a constant force is defined as

$$
\mathrm{C}_{F} \equiv\left(\frac{\Delta E}{\Delta \dot{q}}\right)_{F}=\left(\frac{\partial U}{\partial \dot{q}}\right)_{F}-F\left(\frac{\partial \mathrm{q}}{\partial \dot{q}}\right)_{F} .
$$

2. Second Law. There are processes that satisfy the first law but are not observed in nature. The purpose of the dynamic second law is to incorporate such experimental facts into the model of dynamics.

The statement of the second 1 aw is made using the axiomatic statement provided by the Greek mathematician Caratheodory, who presented an axiomatic deve1opment of the second law of thermodynamics that may be applied to a system of any number of variables. The second law may then be stated as follows:

In the neighborhood (however close) of any equilibrium state of a system of any number of dynamic coordinates, there exist states that cannot be reached by reversible $E$ - conservative $(\bar{d} E=0)$ processes.

When the variables are thermodynamic variables, the $E$-conservative processes are known as adiabatic processes.

A reversible process is one that is performed in such a way that, at the conclusion of the process, both the system and the local surroundings may be restored to their initial states without producing any change in the rest of the universe.

Consider a system whose independent coordinates are a generalized displacement denoted $q$, a generalized velocity $\dot{q}$ (with $\dot{q} \equiv d q / d t$ ), and a generalized force $F$. It can be shown that the $E$-conservative curve comprising all equilibrium states accessible from the initial state, $i$, may be expressed by

$$
\sigma(\dot{q}, q)=\text { constant }
$$


where $\sigma$ represents some as yet undetermined function. Curves corresponding to other initial states would be represented by different values of the constant.

Reversible $E$-conservative curves cannot intersect, for if they did, it would be possible, as shown in Fig. 1, to proceed from an initial equilibrium state $i$, at the point of intersection, to two different firal states $f_{1}$ and $f_{2}$, having the same $q$, along reversible $E$-conservative paths, which is not allowed by the second law.

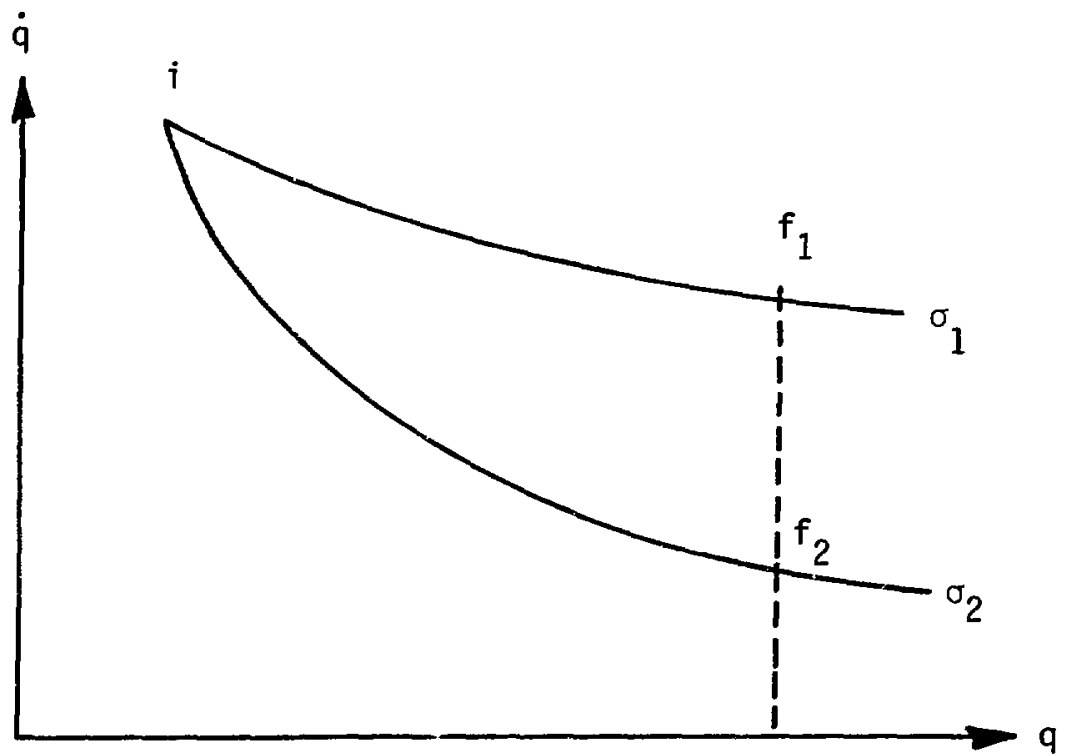

Fig. 1

If two reversible E-conservative curves could intersect, it would be possible to violate the second law by performing the cycle $i, f_{1}, f_{2}, i$.

When the system can be described with only two independent variables, such as on the $E$-conservative curve, then if these variables are $\dot{q}$ and $q$, and $F$ is a generalized force,

$$
\overline{\mathrm{d}} E=\mathrm{d} U-F \mathrm{dq}
$$

Regarding $U=U(\dot{q}, q)$, then

$$
\overline{\mathrm{d}} E=\left(\frac{\partial U}{\partial \dot{q}}\right)_{\mathrm{q}} \mathrm{dq}+\left[\left(\frac{\partial U}{\partial q}\right)_{\dot{q}}-F\right] d q
$$


where $\left(\frac{\partial U}{\partial \dot{q}}\right)_{q}, F$, and $\left(\frac{\partial U}{\partial q}\right)_{\dot{q}}$ are functions of $\dot{q}$ and $q$.

An E-conservative process for this system is

$$
\left(\frac{\partial U}{\partial \dot{q}}\right)_{q} d \dot{q}+\left[\left(\frac{\partial U}{\partial q}\right)_{\dot{q}}-F\right] d q=0 .
$$

Solving for $\mathrm{dq} / \mathrm{dq}$ yields

$$
\frac{\mathrm{d} \dot{q}}{\mathrm{dq}}=\frac{-\left[\left(\frac{\partial U}{\partial \mathrm{q}}\right)_{\dot{q}}-F\right]}{\left(\frac{\partial U}{\partial \dot{q}}\right)_{q}} .
$$

The right hand member is a function of $\dot{q}$ and $q$, and therefore the derivative $\mathrm{d} \dot{q} / \mathrm{dq}$, representing the slope of a E-conservative curve on a $(\dot{q}, q)$ diagram, is known at all poirits. Equatiori (2) has therefore a solution consisting of a family of curves, see Fig. 2, and the curve through any one point may be written

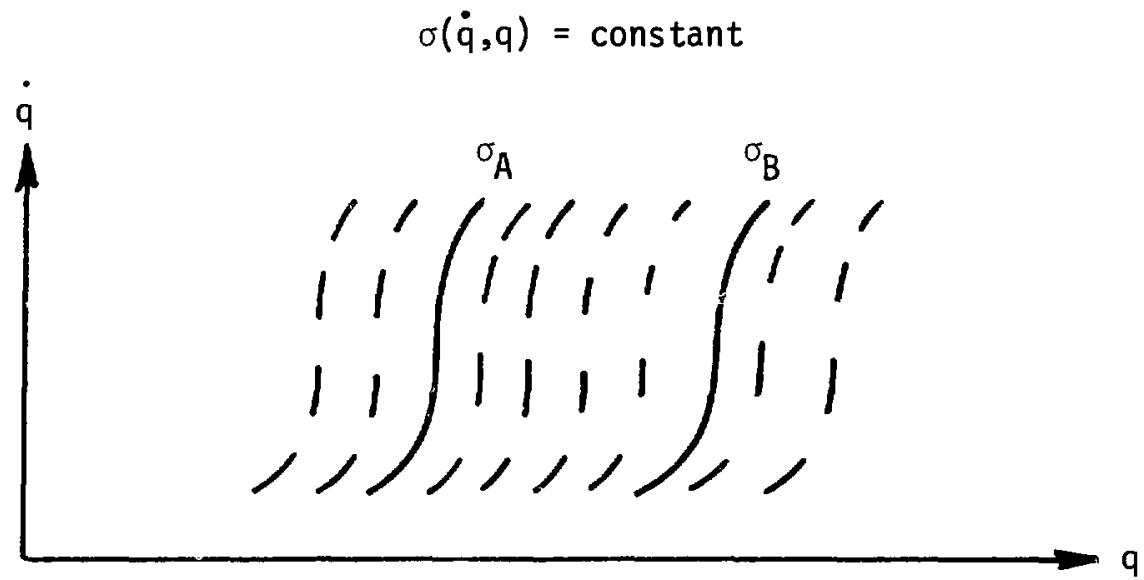

Fig. 2.

The first law, through Eq. (2) fills the $(\dot{q}, q)$ space with slopes specified at each point. The $\sigma$ curves represent the solution curves whose tangents are the required slopes. The second law requires that these curves do not intersect.

A set of curves is obtained when different values äre assigned to the constant. The existence of the family of curves $\sigma(\dot{q}, q)=$ constant, generated by $5 q$. (2), representing reversible $E$-conservative processes, follows from the fact that there are only two independent variables and not from any law of physics. Thus 
it can be seen that the first law may be satisfied by any of these $\sigma=$ constant curves. The axiom requires that these curves do not intersect. Therefore the axiom, together with the first law, leads to the conclusion that through any arbitrary initial-state point, all reversible $E$-conservative processes 1 ie on a curve, and $E$-conservative curves through other initial states determine a family of non-intersecting curves.

To see the results of this conclusion consider a system whose coordinates are the generalized velocity $\dot{q}$, the generalized displacement $q$ and the genera?ized force $F$. The first law is

$$
\overline{\mathrm{d}} E=\mathrm{d} U-F \mathrm{dq}
$$

where $U$ and $F$ are functions of $\dot{q}$ and $q$. Since the $(\dot{q}, q)$ surface is subdivided into a family of non-intersecting $E$-conservative curves

$$
\sigma(\dot{q}, q)=\text { constant }
$$

where the constant can take on various values $\sigma_{1}, \sigma_{2}, \ldots$, any point on the surface may be determined by specifying the value of $\sigma$ along with $q$ so that $U$, as well as $F$, may be regarded as functions of $\sigma$ and $q$. Then

$$
\mathrm{d} U=\left(\frac{\partial U}{\partial \sigma}\right)_{q} \mathrm{~d} \sigma+\left(\frac{\partial U}{\partial q}\right)_{\sigma} \mathrm{dq}
$$

and

$$
\overline{\mathrm{d}} E=\left\langle\frac{\partial U}{\partial \sigma}\right)_{\mathrm{q}} \mathrm{d} \sigma+\left[\left(\frac{\partial U}{\partial q}\right)_{\sigma}-F\right] \mathrm{dq} .
$$

Since $\sigma$ and $q$ are independent variables this equation must be true for all values of $d \sigma$ and $d q$.

Suppose $d \sigma=0$ and $d q \neq 0$. The provision that $d \sigma=0$ is the provision for an $E$-conservative process in which $\bar{d} E=0$. Therefore, the coefficient of $d q$ must vanish. Then, in order for $\sigma$ and $q$ to be independent and for $\bar{d} E$ to be zero when $d \sigma$ is zero, the equation for $\bar{d} E$ must reduce to

$$
\overline{\mathrm{d}} E=\left\langle\frac{\partial U}{\partial \sigma}\right\rangle_{\mathrm{q}} \mathrm{dq},
$$


with

$$
\left(\frac{\partial U}{\partial q}\right)_{\sigma}=F \text {. }
$$

Defining a function $\lambda$ by

$$
\lambda \equiv\left(\frac{\partial U}{\partial \sigma}\right)_{q} \text {, }
$$

then

$$
\overline{\mathrm{d}} E=\lambda \mathrm{d} \sigma,
$$

where

$$
\lambda=\lambda(\sigma, q) \text {. }
$$

Now, in general, an infinitesimal of the type

$$
P d x+Q d y+R d z+\ldots,
$$

known as a linear differential form, or a Pfaffian expression, when it involves three or more independent variables, does not admit of an integrating factor. It is only becaure of the existence of the axiom that the differential form for $\bar{d} E$ referring to a physical system of any number of independent coordinates possess an integrating factor.

Two infinitesimally neighboring reversible E-conservative curves are shown in Fig. 3. One curve is characterized by a constant value of the function $\sigma_{A}$, and the other by a slightly different value $\sigma_{A}+d \sigma=\sigma_{B}$. In any process represented by a displacement along either of the two $E$-conservative curves $\bar{d} E=0$. When i reversible process connects the two $E$-conservative curves, energy $\overrightarrow{\mathrm{d}} E=$ $\lambda$ do is transferred.

The various infinitesimal processes that may be chosen to connect the two neighboring reversible $E$-conservative curves, shown in Fig. 3 , involve the same change of $\sigma$ but take place at different $\lambda$. In general $\lambda$ is a function of $\dot{q}$ and q. However, it is obvious that $\lambda$ may be expressed as a function of $\sigma$ and $\dot{q}$. To 


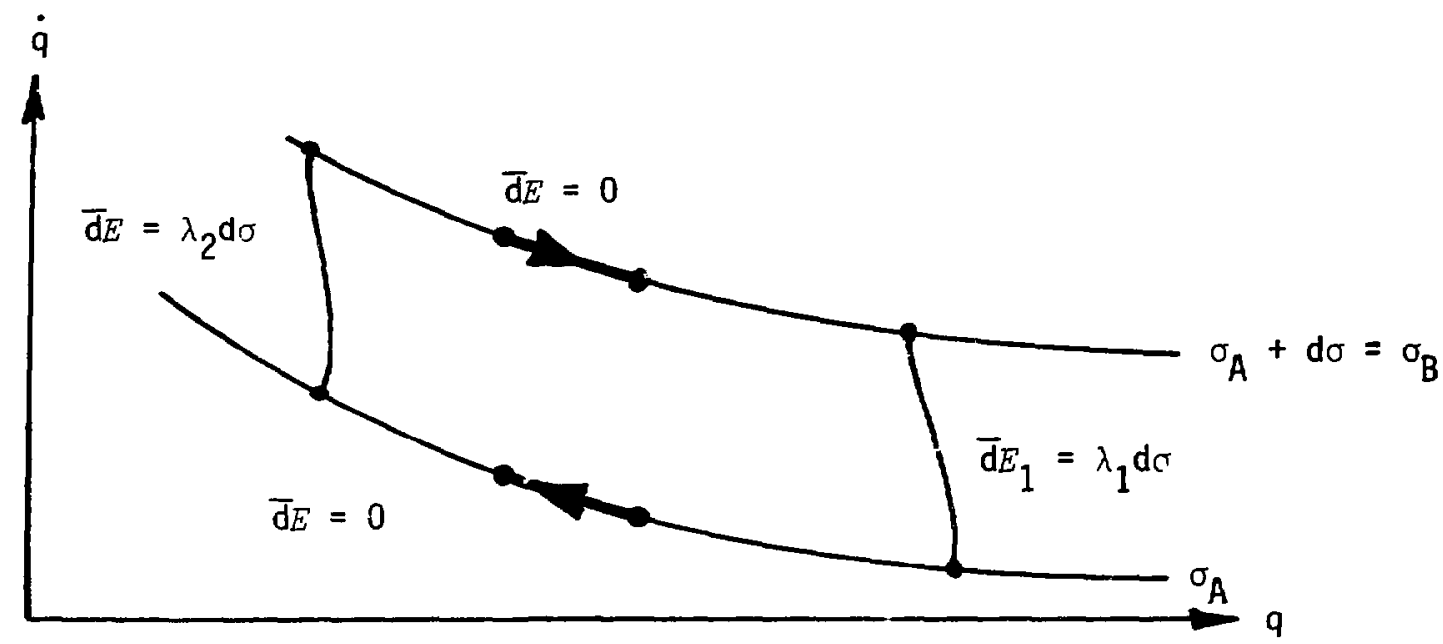

Fig. 3.

Two reversible E-conservative curves, infinitesimally close. When the process is represented by a curve connecting the $E$-conservative curves, energy $d E=\gamma d \sigma$ is transferred.

find the velocity dependence of $\lambda$ consider two systems, one and two, such that in the first system there are two independent coordinates $\dot{q}$ and $q$ and the $E$ conservative curves are specified by different values of the function $\sigma$ of $\dot{q}$ and q. When energy $\bar{d} E$ is transferred, $\sigma$ changes by $d \sigma$ and $\bar{d} E=\lambda d \sigma$ where $\lambda$ is a function of $\sigma$ and $\dot{q}$.

The second system has two independent coordinates $\dot{q}$, and $\hat{q}$ and the $E$ conservative curves are specified by different values of the function $\hat{\sigma}$ of $\dot{q}$ and $\hat{\mathrm{q}}$. When $\bar{d} E$ is transferred, $\hat{\sigma}$ changes by $\mathrm{d} \hat{\sigma}$ and $\bar{d} \hat{E}=\hat{\lambda} \mathrm{d} \hat{\sigma}$ where $\hat{\lambda}$ is a function of $\hat{\sigma}$ and $\dot{q}$.

The two systems are related through the coordinate $\dot{q}$ in that both systems make up a composite system in which there are three independent coordinates $\dot{q}$, $q$, and $\hat{q}$ and the $E$-conservative curves are specified by different values of the function $\sigma_{c}$ of these independent variables.

Since $\sigma=\sigma(\dot{q}, q)$ and $\hat{\sigma}=\hat{\sigma}(\dot{q}, \hat{q})$, using the equations for $\sigma$ and $\hat{\sigma}, \sigma_{c}$ may be regarded as a function of $\dot{q}, \sigma$ and $\hat{\sigma}$.

For an infinitesimal process between two neighboring $E$-conservative surfaces specified by $\sigma_{c}$ and $\sigma_{c}+d \sigma_{c}$, the energy transferred is $\bar{d} E_{c}=\lambda_{c}$ d $\sigma_{c}$ where $\lambda_{c}$ is also a function of $\dot{q}, \sigma$ and $\hat{\sigma}$. Then 


$$
d \sigma_{c}=\frac{\partial \sigma_{c}}{\partial \dot{q}} d \dot{q}+\frac{\partial \sigma_{c}}{\partial \sigma} d \sigma+\frac{\partial \sigma_{c}}{\partial \hat{\sigma}} d \hat{\sigma}
$$

Now suppose that in a process there is a transfer of energy $\bar{d} E_{c}$ between the composite system and an external reservoir with energies $\overline{\mathrm{d}} \hat{E}$ and $\overline{\mathrm{d}} E$ being transferred, respectively, to the first and second systems, then

$$
\overline{\mathrm{d}} E_{\mathrm{c}}=\overline{\mathrm{d}} E+\overline{\mathrm{d}} \hat{E}
$$

and

$$
\lambda_{c} d \sigma_{c}=\lambda d \sigma+\hat{\lambda} d \hat{\sigma}
$$

or

$$
\mathrm{d} \sigma_{c}=\frac{\lambda}{\lambda_{c}} \mathrm{~d} \sigma+\frac{\hat{\lambda}}{\lambda_{c}} \mathrm{~d} \hat{\sigma}
$$

Comparing Eqs. (3) and (4) for $\mathrm{d \sigma}_{\mathrm{c}}$ then

$$
\frac{\partial \sigma_{c}}{\partial \dot{q}}=0
$$

Therefore $\sigma_{c}$ does not depend on $\dot{q}$, but only on $\sigma$ and $\hat{\sigma}$. That is

$$
\sigma_{c}=\sigma_{C}(\sigma, \hat{\sigma})
$$

Again comparing the two expressions for $d \sigma_{c}$

$$
\frac{\lambda}{\lambda_{c}}=\frac{\partial \sigma_{c}}{\partial \sigma} \text { and } \frac{\hat{\lambda}}{\lambda_{c}}=\frac{\partial \sigma_{c}}{\partial \hat{\sigma}}
$$

therefore the two ratios $\lambda / \lambda_{c}$ and $\hat{\lambda} / \lambda_{c}$ are also independent of $\dot{q}, q$ and $\hat{q}$. These two ratios depend only on the $\sigma$ 's, but each separate $\lambda$ must depend on the velocity as well (for example, if $\lambda$ depended only on $\sigma$ and on nothing else, the $\bar{d} E=\lambda d \sigma$ would equal $f(\sigma)$ do which is an exact differential). In order for each $\lambda$ to depend on the velocity and at the same time for the ratios of the $\lambda$ 's to depend 
only on the $\sigma^{\prime} s$, the $\lambda^{\prime} s$ must have the following structure:

$$
\begin{aligned}
& \lambda=\phi(\dot{q}) f(\sigma), \\
& \hat{\lambda}=\phi(\dot{q}) \hat{f}(\hat{\sigma}),
\end{aligned}
$$

and

$$
\lambda_{c}=\phi(\dot{q}) g(\sigma, \hat{\sigma}) .
$$

(The quantity $\lambda$ cannot contain $q$, nor can $\hat{\lambda}$ contain $\hat{q}$, since $\lambda / \lambda_{c}$ and $\hat{\lambda} / \lambda_{c}$ must be functions of the $\sigma^{\prime} s$ only.)

Referring now only to the first system as representative of any system of any number of independent coordinates, the transferred energy is, from Eq. (5),

$$
\bar{d} E=\phi(\dot{q}) \mathbf{f}(\sigma) d \sigma .
$$

Since $f(\sigma)$ do is an exact differential, the quantity $1 / \phi(\dot{q})$ is an integrating factor for $\bar{d} E$. It is an extraordinary circumstance that not only does an integrating factor exist for the $\overline{\mathrm{d}} E$ of any system, but this integrating factor is a function of velocity only and is the same function for all systems.

The fact that a system of two independent variables has a $\bar{d} E$ that always admits an integrating factor regardless of the axiom is interesting, but its importance in physics is not established until it is shown that the integrating factor is a function of velocity only and that it is the same function for all systems.

3. The Absolute Velocity. The universal character of $\phi(\dot{q})$ makes it possible to define an absolute velocity. Consider a system of two independent variables $q$ and $\dot{q}$, for which two constant velocity curves and $E$-conservative curves are shown in Fig. 4. Suppose there is a constant velocity transfer of energy $E$ between the system and an external reservoir at the velocity $\dot{q}$, from a state $b$, on a $E$-conservative curve characterized by the value $\sigma_{1}$, to another state $c$, on another $E$-conservative curve specified by $\sigma_{2}$. Then since

$$
\bar{d} E=\phi(\dot{q}) f(\sigma) d \sigma,
$$


it is seen that

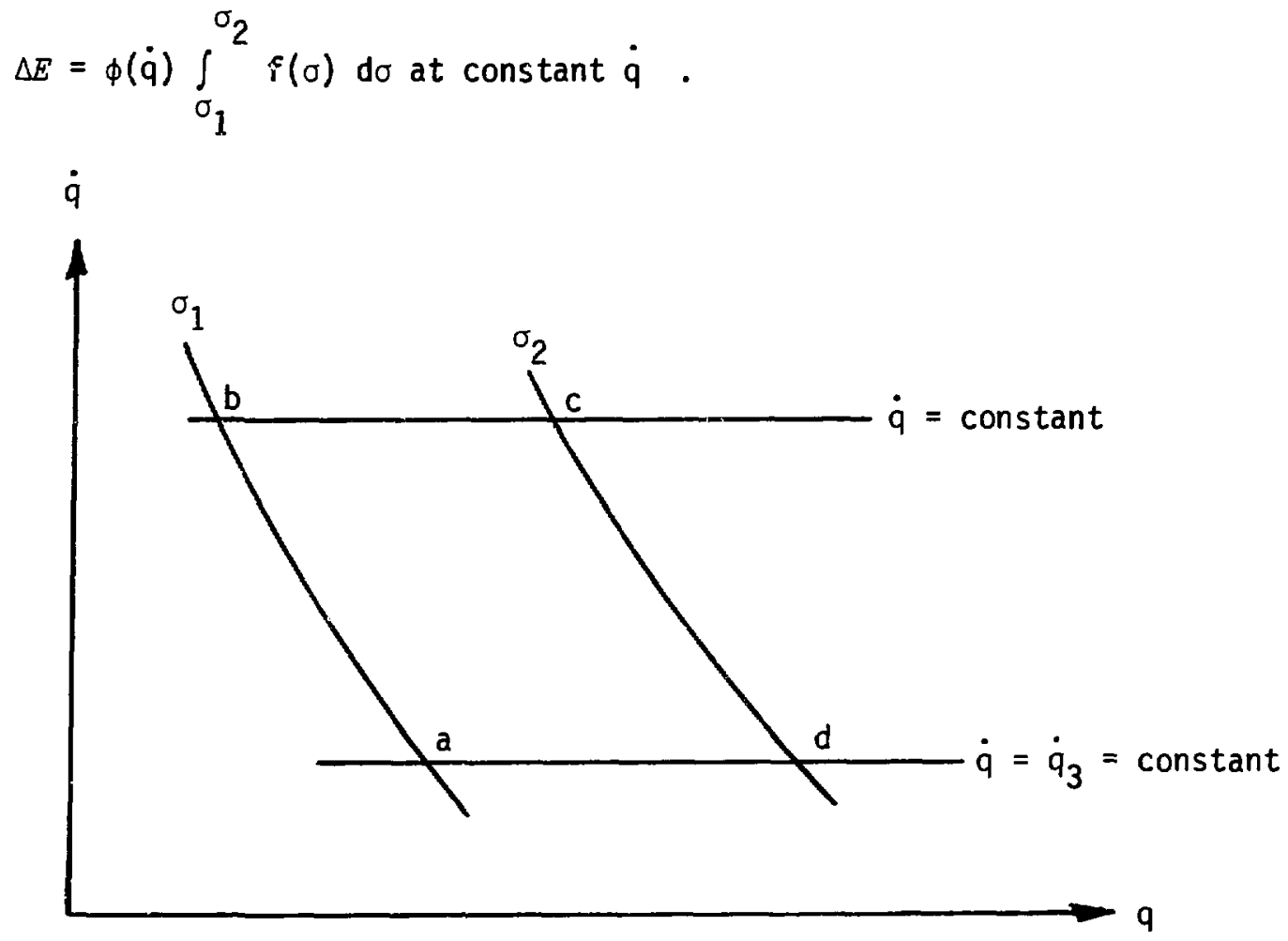

Fig. 4 .

Two constant velocity energy transfers, $E$ at $\dot{q}$ from $b$ to $c$ and $E_{3}$ at $\dot{q}_{3}$ from a to $d$, between the same two conservative curves $\sigma_{1}$ and $\sigma_{2}$.

For any constant velocity process between two other points a to $d$, at a velocity $\dot{q}_{3}$ between the same $E$-conservative curves the energy transferred is $\Delta E\left(\dot{q}_{3}\right)=\Delta E_{3}=\phi\left(\dot{q}_{3}\right) \int_{\sigma_{1}}^{\sigma_{2}} f(\sigma)$ do at constant $\dot{q}_{3}$.

Taking the ratio of

$$
\frac{\Delta E}{\Delta E_{3}}=\frac{\phi(\dot{q})}{\phi\left(q_{3}\right)}=\frac{\text { a function of the velocity at which } \Delta E \text { is transferred }}{\text { same function of velocity at which } \Delta E_{3} \text { is transferred }} .
$$

Then the ratio of these two functions is defined by

$$
\frac{\phi(\dot{q})}{\phi(q)_{3}}=\frac{\left.\Delta E \text { (between } \sigma_{1} \text { and } \sigma_{2} \text { at } \dot{q}\right)}{\left.\Delta E_{3} \text { (between } \sigma_{1} \text { and } \sigma_{2} \text { at } \dot{q}_{3}\right)}
$$


or

$$
\Delta E=\left[\frac{\Delta E_{3}}{\phi\left(\dot{q}_{3}\right)}\right] \phi(\dot{q}) \text {. }
$$

By choosing some appropriate velocity $\dot{q}_{3}$ it follows that the energy transferred at constant velocity between two given $E$-conservative curves decreases as $\phi(\dot{q})$ decreases, or the smaller the value of $E$ the lower the corresponding value of $\phi(\dot{q})$. When $\Delta E$ is zero $\phi(\dot{q})$ is also zero. The corresponding velocity $\dot{q}_{0}$ such that $\phi\left(\dot{q}_{0}\right)$ is zero is the "abso?ute velocity." Therefore, if a system undergoes a constant velocity process between two $E$-conservative curves without an exchange of energy, the velocity at which this takes place is called the absolute velocity.

4. The Concept of Entropy. In a system of two independent variables, all states accessible from a given initial state by reversible $E$-conservative processes 1 ie on a $\sigma(\dot{q}, q)$ curve. The entire $(\dot{q}, q)$ space may be conceived as being filled by many nonintersecting curves of this kind, each corresponding to a different value of $\sigma$. In a reversible non-E-conservative process involving a transfer of energy $\bar{d} E$, a system in a state represented by a point lying on a surface $\sigma$ will change until its state point lies on another surface $\sigma+d \sigma$. Then

$$
\bar{d} E=\lambda d \sigma,
$$

where $1 / \lambda$, the integrating factor of $\bar{d} E$, is given by

$$
\lambda=\phi(\dot{q}) f(\sigma)
$$

and therefore

$$
\bar{d} E=\phi(\dot{q}) f(\sigma) d \sigma
$$

or

$$
\frac{\bar{d} E}{\phi(\dot{q})}=f(\sigma) d \sigma .
$$


Since $\sigma$ is an actual function of $\dot{q}$ and $q$, the right-hand member is an exact differential, which may be denoted by $\mathrm{d} S$; and

$$
\mathrm{d} S=\frac{\overline{\mathrm{d}} E}{\phi(\dot{\mathrm{q}})},
$$

where $S$ is the mechanical entropy of the system and the proress is a reversible one.

The dynamic second law may be used to prove the equivalent of Clausius's theorem, which is stated here without proof.

Theorem: In any cyclic transformation throughout which the velocity is defined, the following inequality holds:

$$
\int \frac{\bar{d} E}{\phi(\dot{q})} \leq 0
$$

where the integral extends over one cycle of the transformation. The equality holds if the cyclic transformation is reversible. Then for an arbitrary transformation

$$
\int_{A}^{B} \frac{\bar{d} E}{\phi(\hat{q})} \leq S(B)-S(A)
$$

with the equality holding if the transformation is reversible. The proof of this statement may be seen by letting $R$ and $I$ denote respectively any reversible and any irreversible path joining $A$ to $B$, as shown in Fig. 5 .

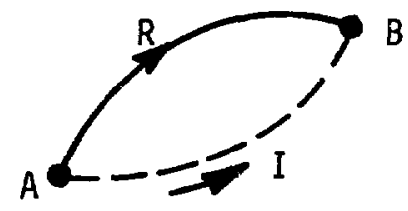

Fig. 5 .

For path $R$ the assertion holds by definition of $S$. Now consider the cyclic transformation made up of I plus the reverse of R. From Clausius' theorem 


$$
\int_{\mathrm{I}} \frac{\overline{\mathrm{d}} E}{\phi}-\int_{\mathrm{R}} \frac{\overline{\mathrm{d}} E}{\phi} \leq 0
$$

or

$$
\int_{\mathrm{I}} \frac{\overline{\mathrm{d}} E}{\phi}-\int_{\mathrm{R}} \frac{\overline{\mathrm{d}} E}{\phi} \equiv S(\mathrm{~B})-S(\mathrm{~A})
$$

Another result of the dynamic second law is that the mechanical entropy of an isolated $(\bar{d} E=0)$ system never decreases. This can be seen since an isolated system cannot exchange energy with the external world since $\overrightarrow{\mathrm{d}} E=0$ for any transformation. Then by the previous property of the entropy,

$$
S(B)-S(A) \geq 0
$$

where the equality holds if the transformation is reversible.

One consequence of the second law is that of all the possible transformations from one state $A$ to another state $B$ the one defined as the change in the entropy is the one for which the integral

$$
I \equiv \int_{A}^{B} \frac{\bar{d} E}{\phi}
$$

is a maximum. Thus

$$
S(B)-S(A) \equiv \operatorname{maximum} I=\max \int_{A}^{B}\left(\frac{1}{\phi} \frac{\bar{d} E}{d \tau}\right) d \tau,
$$

where $\tau$ is a parameter that indicates position along the path from $A$ to $B$, or

$$
S(B)-S(A)=\max \int_{A}^{B}\left(\frac{1}{\phi} \frac{d U}{d \tau}-\frac{E}{\phi} \frac{d q}{d \tau}\right) d \tau \text {. }
$$

If

$$
U=U\left(\tau, q, \dot{q}, \frac{d \dot{q}}{d \tau}\right)
$$


where $\dot{q}^{i}=d q^{i} / d \tau$, then the change in the entropy is given by the integral

$$
\Delta S=\int_{\mathrm{A}}^{\mathrm{B}}\left(\frac{1}{\phi} \frac{\mathrm{d} U}{\mathrm{~d} \tau}-\frac{F}{\phi} \frac{\mathrm{dq}}{\mathrm{d} \tau}\right) \mathrm{d} \tau .
$$

The $\dot{q}$ and $q$ which maximize $\Delta S$ will be denoted as $\dot{x}$ and $x$ then, with

$$
\begin{aligned}
& U=U(x, \dot{x}) \\
& F=F(x, \dot{x}) \\
& \phi=\phi(\dot{x})
\end{aligned}
$$

the $x$ and $\dot{x}$ are given by the solution of the system of equations

$$
\begin{aligned}
& \frac{d}{d \tau}\left(\frac{\partial G}{\partial x^{\prime}}\right)-\frac{\partial G}{\partial x}=0 \\
& \frac{d}{d \tau}\left(\frac{\partial G}{\partial \dot{x}^{\prime}}\right)-\frac{\partial G}{\partial \dot{x}}=0
\end{aligned}
$$

where

$$
G=\left(\frac{1}{\phi}\right)\left[\frac{\partial U}{\partial \tau}-F \frac{\mathrm{dx}}{\mathrm{d} \tau}\right] \text { and } \quad \mathrm{x}^{\prime}=\frac{\mathrm{dx}}{\mathrm{d} \tau} \quad \text { and } \quad \dot{x}^{\prime}=\frac{\mathrm{d} \dot{\mathrm{x}}}{\mathrm{d} \tau} \text {. }
$$

Thus the dynamic second law provides an answer to the question that is not contained within the scope of the first law: In what direction does a process take place? The answer is that a process always takes place in such a direction as to cause an increase of the mechanical entropy in the universe. In the case of an isolated system, it is the entropy of the system that tends to increase. To find out, therefore, the equilibrium state of an isolated one-dimensional system, it is necessary merely to express the entropy as a function of $q$ and $\dot{q}$ and to apply the usual rules of calculus to render the function a maximum. When ihe system is not isolated, there are other entropy changes to be taken into account.

5. Third Law. The dynamic second law enables the mechanical entropy of a system to be defined up to an arbitrary additive constant. The definition 
depends on the existence of a reversible transformation connecting an arbitrarily chosen reference state 0 to the state under consideration. Such a reversible transformation always exists if both 0 and $A 1$ ie on one sheet of the state surface. If two different systems are considered, the equation of the state surface may consist of several disjoint sheets. In such cases the kind of reversible path previously mentioned may not exist. Therefore the second law does not uniquely determine the difference in entropy of two states $A$ and $B$, if A defines a state of one system and B the state of another. For this determination a dynamic third law is needed. The dynamic third law may be stated, "The mechanical entropy of a system at the absolute velocity is a universal constant, which may be taken to zero." In the case of a purely thermodynamic system the absolute quantity is the absolute zero temperature, while for a mechanical system the absolute quantity is the absolute velocity.

The dynamic third law implies that any energy capacity of a system must vanish at the absolute velocity. To see this, let $R$ be any reversible path connecting a state of the system at the absolute velocity $\dot{q}_{0}$ to the state $A$, whose entropy is to be found. Let $C_{R}(\dot{q})$ be the energy capacity of the system along the path $R$. Then, by the second law,

$$
S(A)=\int_{\dot{q}_{0}}^{\dot{q}_{A}} C_{R}(\dot{q}) \frac{d \dot{q}}{\phi(\dot{q})} .
$$

But according to the third law,

$$
S(A)+0
$$

as

$$
\dot{q}_{A} \rightarrow \dot{q}_{0}
$$

Hence it follows that

$$
\begin{aligned}
C_{R}(\dot{q}) & \rightarrow 0 \\
q & \rightarrow \dot{q}_{0} .
\end{aligned}
$$


In particular, $C_{R}$ may be $C_{q}$ or $C_{F}$.

The statement of the third law above reflects the restriction to mechanical work terms. A general statement of the third law that is independent of the number or type of variables is "The generalized entropy of the system, when the integrating factor vanishes, is a universal constant, which may be taken to be zero."

B. General Maxwell and Energy Relations

In thermodynamics a discussion of equilibrium and stability conditions is best dorie if the enthalpy, Helmholtz's, and Gibb's functions are defined first. Therefore, the mechanical analogues of these functions are defined here.

Each branch of physics such as thermodynamics and particle dynamics has its own developed procedures. If both branches can be described by the same basic dynamic laws, then the procedures developed in thermodynamics may prove to be useful in particle dynamics and vice versa. Once the mechanical enthalpy, mechanical Helmholtz's and mechanical Gik's' functions are defined, it is then easy to write down the resulting mechanical Maxwell and mechanical energy capacity relations.

To begin the development of the Maxwell relations, the mechanical entropy was defined as

$$
\mathrm{d} S \equiv \frac{\overline{\mathrm{d}} E}{\phi(\hat{q})}
$$

Then, since $\overline{\mathrm{d}} E=\mathrm{d} U-F \mathrm{dq}$.

$$
\mathrm{d} S=\frac{\mathrm{d} U}{\phi}-\frac{F}{\phi} \mathrm{dq},
$$

where

$$
\mathrm{d} U=\phi(\dot{q}) \mathrm{d} S+F \mathrm{dq}
$$

Define the mechanical enthalpy as

$$
H \equiv U-F q \quad ;
$$


then

$$
\mathrm{d} H=\phi(\dot{\mathrm{q}}) \mathrm{d} S-\mathrm{qd} F .
$$

Therefore

$$
\left(\frac{\partial F}{\partial S}\right)_{F}=\phi(\dot{q}) \text { and }\left(\frac{\partial H}{\partial F}\right)_{S}=-q \text {. }
$$

The mecharical Helmholtz's function can be defined as

$$
K \equiv U-\phi(\dot{q}) S \text {, }
$$

and

$$
\mathrm{d} K=\mathrm{d} U-\frac{\mathrm{d} \phi(\dot{q})}{\mathrm{d} \dot{q}} S \mathrm{~d} \dot{q}-\phi(\dot{\mathrm{q}}) \mathrm{d} S,
$$

or, with

$$
\begin{aligned}
& \phi^{\prime}(\dot{q})=\frac{d \phi}{d q}, \\
& d K=-S \phi^{\prime}(\dot{q}) d \dot{q}-F d q .
\end{aligned}
$$

This leads to

$$
\left(\frac{\partial K}{\partial \dot{q}}\right)_{q}=-S \phi^{\prime}(\dot{q}) \quad \text { and } \quad\left(\frac{\partial K}{\partial q}\right)_{\dot{q}}=\phi(\dot{q}) F \text {. }
$$

The mechanical Gibb's function may be defined as

$$
G \equiv H-\phi(\dot{q}) S \quad ;
$$

then

$$
\mathrm{d} G=-\phi^{\prime}(\dot{\mathrm{q}})_{S} \mathrm{~d} \dot{\mathrm{q}}+\mathrm{qd} F,
$$


so that

$$
\left(\frac{\partial G}{\partial \dot{q}_{F}}\right)_{F}=-\phi^{\prime}(\dot{q}) S \quad \text { and } \quad\left(\frac{\partial G}{\partial F}\right)_{\dot{q}}=q
$$

From the differential Eqs. (7), (8), (9), and (10) the Maxwell relations for a mechanical system may be written:

$$
\begin{aligned}
& \phi^{\prime}(\dot{q})\left(\frac{\partial \dot{q}}{\partial q}\right)_{S}=\left(\frac{\partial F}{\partial S}\right)_{q} \\
& \phi^{\prime}(q)\left(\frac{\partial \dot{q}}{\partial F}\right)=-\left(\frac{\partial q}{\partial S}\right)_{F} \\
& \phi^{\prime}(\dot{q})\left(\frac{\partial S}{\partial q}\right)_{\dot{q}}=-\left(\frac{\partial F}{\partial \dot{q}}\right)_{q} \\
& \phi^{\prime}(\dot{q})\left(\frac{\partial S}{\partial F}\right)_{\dot{q}}=\left(\frac{\partial q}{\partial \dot{q}}\right)_{F} .
\end{aligned}
$$

The energy capacity at the position $q$ can be defined as

$$
C_{q} \equiv\left(\frac{\bar{d} E}{\dot{q}}\right)_{q}=\phi(\dot{q})\left(\frac{\partial S}{\partial \dot{q}}\right)_{q} \text {. }
$$

Define the energy capacity with a constant force as

$$
C_{F} \equiv\left(\frac{\bar{d} E}{d \bar{q}}\right)_{F}=\phi(\dot{q})\left(\frac{\partial S}{\partial \dot{q}}\right)_{F},
$$

then

$$
\left(C_{q}-C_{F}\right)=\frac{\phi(\dot{q})}{\phi^{\prime}}\left(\frac{\partial q}{\partial \dot{q}}\right)_{F}\left(\frac{\partial F}{\partial \dot{q}}\right)_{q} \text {, }
$$

and

$$
\frac{C_{F}}{C_{q}}=\frac{\left(\frac{\partial F}{\partial q}\right)_{S}}{\left(\frac{\partial F}{\partial q}\right)_{\dot{q}}} .
$$




\section{Equilibrium and Stability Conditions}

The three generalized laws have been formulated and a few . .lts of these laws have been seen. The next step is to derive the stability conditions to obtain the quadratic forms necessary for a metric. In the process of deriving the equilibrium conditions and in turn the stability conditions other state functions are used. These functions may be defined briefly here as:

Mechanical enthalpy $(H): H \equiv U-F q$

Mechanical Helmholtz function $(K): K \equiv U-\phi(\dot{q}) S$,

and

Mechanical Gibbs function $(G): G \equiv H-\phi(\dot{q}) S$.

The derivation of the equilibrium and stability conditions is identical to the derivation of the thermodynamic equilibrium and stability conditions with the variables changed to represent the mechanical variables $\dot{q}, q, S$ and $F$ instead of the thermodynamic variables $T, V, S$ and $P$.

1. Equilibrium Conditions. To establish the criteria for equilibrium, consider Clausius's theorem

$$
\int_{A}^{B} \frac{\bar{d} E}{\phi}-\int_{A}^{B} \frac{\bar{d} E}{\phi} \leq 0,
$$

or

$$
\int_{\mathrm{A}}^{\mathrm{B}} \frac{\overline{\mathrm{d}} E}{\phi} \leq \int_{\mathrm{A}}^{\mathrm{B}} \frac{\mathrm{d} E}{\phi} \equiv S(\mathrm{~B})-S(\mathrm{~A}) .
$$

For an $E$-conservative system $\overline{\mathrm{d}} E=0$, then

$$
\Delta S \geq 0
$$

or 


$$
S(\mathrm{~B}) \geq S(\mathrm{~A})
$$

Therefore the mechanical entropy tends toward a maximum so that spontaneous changes in an $E$-conservative system will always be in the direction of increasing mechanical entropy.

Now by the first law

$$
\Delta E=\Delta U-F \Delta q
$$

Therefore

$$
\phi \Delta S \geq \Delta U-F \Delta \mathrm{q},
$$

which is analogous to the Clausius inequality in thermodynamics.

Now consider a virtual displacement $(U, q) \rightarrow(U+\delta U, q+\delta q)$, which implies a variation $S \rightarrow S+\delta S$ away from equilibrium. The restoration of equilibrium from the varied state $(U+\delta U, q+\delta q) \rightarrow(U, q)$ will then certainly be a spontaneous process, and by the Clausius inequality

$$
\phi(-\delta S)>-(\delta U-F \delta q)
$$

Hence, for variations away from equipoise, the general inequality

$$
\delta U-F \delta q-\phi \delta S>0
$$

must hold. The inequality sign is reversed from the sign in Clausius' inequality because hypothetical variations $\delta$ away from equilibrium are considered rather than real changes toward equilibrium.

In a spontaneous process,

$$
\phi \Delta S \geq \Delta E_{\text {rev }}=\Delta U+\text { work done by the system. }
$$

The "work" consists of two parts. One part is the work done by the negative of the force $F$. It may be positive or negative, but it is inevitable. Only the rest is free energy, which is available for some useful work. This latter part 
may be written as

$$
A=\Delta E_{\text {rev }}-\Delta U+F \Delta q
$$

The maximum of $A$ is

$$
A_{\max }=\phi \Delta S-\Delta U+F \Delta q,
$$

which is obtained when the process is conducted reversibly.

The least work, $\delta A_{\min }$, required for a displacement from equilibrium must be exactly equal to the maximum work in the converse process whereby the system proceeds spontaneolisly from the "displaced" state to equilibrium (otherwise a perpetual motion machine may be constructed). Corresponding to Eq. (13) then,

$$
\delta A_{\min }=\delta U-F \delta q-\phi \delta S .
$$

The equilibrium criteria may then be expressed as

$$
\delta A_{\min } \geq 0
$$

In words: At equilibrium the mechanical free energy is a minimum. Any displacement from this state requires work.

2. Stability Conditions. To decide whether or not an equilibrium is stable, the inequality sign in Eq. (12) must be ensured. The conditions for stability may take different forms depending upon which variables are taken as the independent variables.

To derive the stability conditions when $q$ and $S$ are taken as the independent variables consider the terms of second order in small displacements beginning with the general condition

$$
\delta U-F \cup q-\phi \delta S>0 .
$$

Choose $U=U(q, S)$, which, because of the identity

$$
\mathrm{d} S=\frac{\mathrm{d} U}{\phi}-\frac{F}{\phi} \mathrm{dq}
$$


or

$$
\phi \mathrm{d} S=\mathrm{d} U-F \mathrm{dq},
$$

is a natural choice of the independent variables, and expand $\delta U$ in powers of $\delta q$ and $\delta S$

$$
\begin{aligned}
\delta U= & \phi \delta S+F \delta q+\frac{1}{2}\left(\frac{\partial^{2} U}{\partial q^{2}}\right) \delta q^{2}+2 \frac{\partial^{2} U}{\partial q \delta S} \delta q \delta S+\frac{\partial^{2} U}{\partial S^{2}} \delta S^{2} \\
& + \text { terms of third order } \ldots
\end{aligned}
$$

The inequality (12) then shows that in (14),

$$
\text { second order terms }+ \text { third order terms }+\ldots>0 \text {. }
$$

Retaining only the second order terms, the criterion of stability is that a quadratic differential form be positive definite;

$$
\frac{\partial^{2} U}{\partial q^{2}} \delta q^{2}+2 \frac{\partial^{2} U}{\partial q \partial S} \delta q \delta S+\frac{\partial^{2} U}{\partial S^{2}} \delta S^{2}>0
$$

If this is to hold true for arbitrary variations in $\delta q$ and $\delta S$, the coefficients must satisfy the following:

$$
\frac{\partial^{2} U}{\partial q^{2}}>0 ; \frac{\partial^{2} U}{\partial S^{2}} \frac{\partial^{2} U}{\partial q^{2}}-\left(\frac{\partial^{2} U}{\partial q \partial S}\right)^{2}>0 .
$$

When $\dot{q}$ and $q$ are considered to be the independent variables, a quadratic form in $\delta \dot{q}$ and $\delta q$ may be found by using

$$
K=U-\phi S
$$

so that

$$
\delta K=\delta U-\phi \delta S-\frac{d \phi}{d q} S \delta \dot{q}-\frac{d \phi}{d \dot{q}} \delta S \delta \dot{q} .
$$


The terms $\delta S \delta \dot{q}$ cannot be neglected because in Clausius's inequality, which is the actual stability condition, the variations are finite, and therefore, from Eq.

(12) the following is obtained:

$$
\begin{aligned}
& \delta K+\phi \delta S+\frac{d \phi}{d \dot{q}}(S+\delta S) \delta \dot{q}-F \delta q-\phi \delta S>0, \\
& \delta K+\frac{d \phi}{d \dot{q}} S \delta \dot{q}+\frac{d \phi}{d \dot{q}} \delta S \delta \dot{q}-F \delta q>0 .
\end{aligned}
$$

Expanding in powers of $\delta \dot{q}$ and $\delta q$,

$$
\delta K=F \delta q-\frac{d \phi}{d q} S \delta \dot{q}+\frac{1}{2} \frac{\partial^{2} K}{\partial q^{2}} \delta q^{2}+\frac{\partial^{2} K}{\partial q \partial \dot{q}} \delta q \delta \dot{q}+\frac{1}{2} \frac{\partial^{2} K}{\partial q^{2}} \delta q^{2}+\ldots
$$

and

$$
\delta S \delta \dot{q}=\frac{1}{\phi} \frac{\partial U}{\partial \dot{q}} \delta \dot{q}^{2}+\frac{1}{\phi}\left(\frac{\partial U}{\partial q}-F\right) \delta q \delta \dot{q} .
$$

But

$$
\frac{\partial K}{\partial \dot{q}}=\frac{\partial U}{\partial \dot{q}}-\frac{\partial \phi}{\partial \dot{q}} S-\frac{\phi}{\phi} \frac{\partial U}{\partial \dot{q}}=-\frac{d \phi}{d q} S
$$

and

$$
\frac{\partial K}{\partial q}=F
$$

Therefore

$$
\frac{\partial^{2} K}{\partial \dot{q} \partial \dot{q}}=\frac{\partial F}{\partial \dot{q}}=-\frac{\partial \phi}{\partial q}\left(\frac{1}{\phi}\right)\left(\frac{\partial U}{\partial q}-F\right),
$$

and

$$
\frac{\partial^{2} K}{\partial \dot{q}^{2}}=-\frac{\partial^{2} \phi}{\partial \dot{q}^{2}} S-\frac{d \dot{q}}{d q}\left(\frac{1}{\phi}\right) \frac{\partial U}{\partial \dot{q}}
$$

32 
Then

$$
\left(\frac{d \phi}{d \dot{q}}\right) \delta S \delta \dot{q}=-\left(\frac{\partial^{2} \phi}{\partial \dot{q}^{2}} S+\frac{\partial^{2} K}{\partial \dot{q}^{2}}\right)(\delta \dot{q})^{2}-\frac{\partial^{2} K}{\partial \dot{q} \partial \dot{q}} \delta \dot{q} \delta q,
$$

and the quadratic form in $\delta \dot{q}$ and $\delta q$ is

$$
\begin{aligned}
\frac{1}{2} \frac{\partial^{2} K}{\partial q^{2}}(\delta q)^{2} & +\frac{\partial^{2} K}{\partial q \partial^{\dot{q}}} \delta q \delta \dot{q}+\frac{1}{2} \frac{\partial^{2} K}{\partial \dot{q}^{2}}(\delta \dot{q})^{2}-\frac{\partial^{2} K}{\partial \dot{q}^{2}}(\delta \dot{q})^{2} \\
& -\frac{d^{2} \phi}{d \dot{q}^{2}} S(\delta \dot{q})^{2}-\frac{\partial^{2} K}{\partial q \partial \dot{q}} \delta q \delta \dot{q}>0,
\end{aligned}
$$

or

$$
\frac{\partial^{2} K}{\partial q^{2}}(\delta q)^{2}-\left(\frac{\partial^{2} K}{\partial \dot{q}^{2}}+2 \frac{d^{2} \phi}{\partial \dot{q}^{2}} S\right)(\delta \dot{q})^{2}>0 .
$$

Since $\left(\frac{\partial K}{\partial q}\right)_{\dot{q}}=F$, then

$$
\frac{\partial^{2} K}{\partial q^{2}}=\left(\frac{\partial F}{\partial q}\right)_{\dot{q}}>0
$$

Other quadratic forms may be derived by using different independent variables; however, these two quadratic forms will suffice for this development.

\section{Geometry and Field Equations}

There is nothing that specifies which of the quadratic forms coming from the stability conditions should be adopted as the metric. Thus the choice may be based upon simplicity and/or applicability. However, it becomes obvious that if we choose one of the forms using the velocity as our metric and then obtain equations of motion, then the equations of motion will become third order differential equations since the velocity is itself first order and the equations of motion are second order differential equations. 
The fact that these equations of motion will become third order differential equations in time displays a time asymmetry that appears to correspond to nature. However, third order equations are difficult or impossible to solve.

1. Geometry. To avoid the difficulty of third order equations of motion, suppose we adopt the quadratic form of Eq. (15) as the metric for our system. Thus we are adopting a manifold with coordinates of space-mechanical entropy.

It now becomes desirable to extend our system beyond the dimensionality used thus far. Such an extension brings up a question concerning the integrating factor. With one work term the differential of the entropy was written as

$$
\mathrm{d} S=\frac{\overline{\mathrm{d}} E}{\phi}=f(\sigma) \mathrm{d} \sigma .
$$

Then if for each dimension the exchange of energy is denoted by $\bar{d} E_{j}$, then

$$
\mathrm{d} S_{\mathbf{i}}=\frac{\overline{\mathrm{d}} E_{\mathbf{i}}}{\phi_{\mathbf{i}}}=\mathrm{f}_{\mathbf{i}} \mathrm{d} \sigma_{\mathbf{i}}
$$

where there is no summation intended for $f_{j} d \sigma_{j}$. Since each $d S_{j}$ is a perfect differential, then the total change in nechanical entropy may be written as

$$
\mathrm{d} S=\sum_{i} \mathrm{~d} S_{i}=\sum_{i} \frac{\overline{\mathrm{d}} E_{\mathbf{i}}}{\phi_{i}}=\sum_{i} f_{i} \mathrm{~d} \sigma_{i}
$$

However, the question which arises is whether there exists a single integrating factor $\phi$ such that

$$
\mathrm{d} S=\frac{\overline{\mathrm{d}} E}{\phi}=\sum_{\mathbf{i}} \frac{\overline{\mathrm{d}} E_{\mathbf{i}}}{\phi_{\mathbf{i}}}=\sum_{\mathbf{i}} \boldsymbol{f}_{\mathbf{i}} \mathrm{d} \sigma_{\mathbf{i}}
$$

To see this consider the element of work considered before as

$$
\overline{\mathrm{d} W}=\sum_{\mathbf{j}} E_{\mathbf{i}} \mathrm{dq}{ }^{\mathbf{j}} ; \mathbf{i}=1, \ldots, n
$$

Since each $\mathrm{d} U_{\boldsymbol{i}}$ is in itself a perfect differential, then $\mathrm{d} U=\sum_{\mathbf{i}} \mathrm{d} U_{\mathbf{i}}$ so that

$$
\overline{\mathrm{d}} E=\sum_{\mathbf{i}} \mathrm{d} U_{\mathbf{j}}-\sum_{\mathbf{j}} F_{\mathbf{j}} \mathrm{dq} \mathbf{i}^{\mathbf{i}}=\sum_{\mathbf{i}}\left(\mathrm{d} U_{\mathbf{i}}-F_{\mathbf{i}} \mathrm{dq} \mathbf{q}^{\mathbf{j}}\right)
$$


or

$$
\overline{\mathrm{d}} E=\sum_{\mathbf{i}} \overline{\mathrm{d}} E_{\mathbf{i}}
$$

If the system is total $E$-conservative in the sense that

$$
\overline{\mathrm{d}} E=\sum_{\mathbf{i}} \overline{\mathrm{d}} E_{\mathbf{i}}=0
$$

then $\bar{d} E=0$ is a Pfaffian differential equation. This equation is integrable and has an integrating factor $\phi$. The integrability is guaranteed by the dynamic second law since it is impossible to go from one initial state to any neighboring state. Then, just as in the one-dimensional case, the perfect differential follows:

$$
\mathrm{d} S=\frac{\overline{\mathrm{d}} E}{\phi}=\frac{\sum \overline{\mathrm{j}} E_{\boldsymbol{i}}}{\phi} .
$$

But since

$$
\overline{\mathrm{d}} E_{\mathbf{i}}=\phi_{\mathbf{i}} \mathrm{f}_{\mathbf{j}} \mathrm{d} \sigma_{\mathbf{i}}
$$

then

$$
\mathrm{d} S=\sum_{i} \frac{\phi_{i} f_{i} d \sigma_{i}}{\phi}
$$

Now following the same argument presented in Section II.A.2 concerning the composite system,

$$
\overline{\mathrm{d}} E=\lambda \mathrm{d} \sigma
$$

where $\sigma$ is a function of all the $\sigma_{i}$ and the $\dot{q}^{i}$. Therefore since

$$
\bar{d} E_{\mathbf{i}}=\lambda_{\mathbf{i}} \mathrm{d} \sigma_{\mathbf{i}}
$$


then

$$
\overline{\mathrm{d} E}=\sum_{\mathbf{i}} \lambda_{\mathbf{i}}\left\{\frac{\partial \phi_{\mathbf{i}}}{\partial \sigma} \mathrm{d} \sigma+\mathrm{d} \sigma_{\mathbf{i}}\right\}
$$

Now

$$
d \sigma=\sum_{i}\left(\frac{\partial \phi}{\partial \dot{q}^{i}} d \dot{q} \dot{i}+\frac{\partial \sigma}{\partial \sigma_{i}} d \sigma_{i}\right)
$$

so that

$$
\overline{\mathrm{d}} E=\sum_{\mathbf{i}} \overline{\mathrm{d}} E_{\mathbf{i}}
$$

or

$$
\lambda d \sigma=\sum_{\mathbf{i}} \lambda_{\mathbf{i}} \mathrm{d} \sigma_{\mathbf{i}}
$$

and

$$
d \sigma=\sum_{i} \frac{\lambda_{i}}{\lambda} d \sigma_{i}
$$

It follows that the $\partial \sigma / \partial \dot{q}^{j}=0$ and that the ratios $\lambda_{j} / \lambda$ are also independent of the $\dot{q}^{j}$. Therefore the $\lambda$ 's have the form

$$
\begin{aligned}
\lambda_{i} & =\phi f_{i} \\
\lambda & =\phi F\left(\sigma_{1}, \sigma_{2}, \ldots, \sigma_{n}\right)
\end{aligned}
$$

and also

$$
\frac{\mathrm{d} E}{\phi}=\mathrm{Fd} \sigma=\sum_{\mathbf{i}} \mathrm{F} \frac{\lambda_{\mathbf{i}}}{\lambda} \mathrm{d} \sigma_{\mathbf{i}}=\sum_{\mathbf{i}} \frac{\lambda_{\mathbf{i}} \mathrm{d} \sigma_{\mathbf{i}}}{\phi}=\sum_{\mathbf{i}} \mathbf{f}_{\mathbf{i}} \mathrm{d} \sigma_{\mathbf{i}}
$$


The right hand side is a perfect differential and therefore so is the left.

Since $\lambda_{j} / f_{i}$ is an integrating factor and $\lambda / F$ is also an integrating factor, it follows that $\phi\left(\dot{q}^{1}, \dot{q}^{2}, \ldots, \dot{q}^{n}\right)$ is an integrating factor for the $\bar{d} E_{j}$ as well as for $\overline{\mathrm{d} E}-\sum_{\mathbf{i}} \overline{\mathrm{d}} E_{\mathbf{i}}$. Therefore

$$
\mathrm{d} S=\frac{\overline{\mathrm{d}} E}{\phi}=\sum_{\mathbf{i}} \frac{\overline{\mathrm{d}} E_{\mathbf{i}}}{\phi}
$$

The importance of this question may be seen in terms of the difficulty that would be created if a universal integraiting factor could not be found. For then each additional work term would require its own integrating factor determined individually.

Thus assured that an overali integrating factor exists, then the existence of an overall entropy function is guaranteed so that

$$
\mathrm{d} S=\frac{\overline{\mathrm{d}} E}{\phi}=\frac{\mathrm{d} U}{\phi}-\frac{F_{\mathbf{i}}}{\phi} \mathrm{dq}^{i}
$$

for any $i$ and the quadratic form may be extended to include three spatial work terms and thus becomes

$$
\frac{\partial^{2} U}{\partial S^{2}}(\mathrm{~d} S)^{2}+2 \frac{\partial^{2} U}{\partial S \partial q^{\alpha}}(\mathrm{d} S)\left(\mathrm{dq} q^{\alpha}\right)+\frac{\partial^{2} U}{\partial q^{\alpha} \partial q^{\beta}}\left(d q^{\alpha}\right)>0 ; \alpha, \beta=1,2,3 .
$$

Adopting this quadratic form as the metric of a general system whose thermodynamic variables are held fixed, we may then write this metric as

$$
(d s)^{2} \equiv h_{i j} d a^{i} d q^{j} ;(i, j=0,1,2,3),
$$

where the summation convention is used and

$$
h_{i j} \equiv \frac{\partial^{2} U}{\partial q^{i} \partial q^{j}} \text {, }
$$

with $q^{0} \equiv S / F_{0}$, the scaled mechanical entropy for dimensional correctness. 
Thus, the stability conditions provide a metric in the four-dimensional manifold of space-mechanical entropy. However, the existing relativistic theories are theories in a space-time manifold. Therefore, if these theories are to be contained within the dynamic theory, then the space-time manifold must be found within the dynamic theory.

The arc length $s$ in the space-mechanical entropy manifold may be parameterized by choosing

$$
\mathrm{d} s \equiv \dot{\mathrm{q}}_{0} \mathrm{dt} \equiv \mathrm{cdt},
$$

where $\dot{q}_{C} \equiv c$ is the unique velocity appearing in the integrating factor of the second postulate. The metric may now be written as

$$
c^{2}(d t)^{2}=h_{i j} d q^{i} d q^{j} ;(i, j=0,1,2,3) .
$$

Now suppose the systems considered are restricted to only E-conservative systems. Then the principle of increasing mechanical entropy may be imposed in the form of the variational principle

$$
\delta \int \sqrt{\left(d q^{0}\right)^{2}}=0
$$

In order to use this variation principle, Eq. (17) may be expanded, solved for $\left(\mathrm{dq}^{0}\right)$ and squared to arrive at the quadratic form

$$
\begin{aligned}
& \left(d q^{0}\right)^{2}=\left(\frac{1}{h_{00}}\right)\left\{c^{2}(d t)^{2}+2 h_{0 \alpha} A d t d q^{\alpha}-h_{\alpha \beta} d q^{\alpha} d q^{\beta}\right\} . \\
& A \equiv \frac{h_{0 \gamma} \dot{q}^{\gamma}}{h_{00}} \pm \sqrt{\frac{c^{2}}{h_{00}} \frac{h_{\gamma \beta} \dot{q}^{\gamma} \dot{q}^{\beta}}{h_{00}}+\frac{h_{0 \gamma} \dot{q}^{2}}{h_{00}}}
\end{aligned}
$$

with $\dot{q}^{\gamma} \equiv d q^{\gamma} / d t$.

By defining $x^{0} \equiv c t, x^{\alpha}=q^{\alpha} ; \alpha=1,2,3$, then Eq. (18) may be written as

$$
\left(d q^{0}\right)^{2}=\left(\frac{1}{f}\right) \hat{\hat{g}}_{i j} d x^{i} d x^{j} ;(i, j=0,1,2,3)
$$


where $f \equiv h_{00}$. This metric obviously reduces, in the Euclidean limit of constant coefficients, to the metric of Minkowski's space-time manifold of special relativity.

In his General Theory of Relativity, Einstein assumed the space-time manifold to be Riemannian. However, this assumption involves the a priori assumption that the scalar product be invariant. This assumption was later questioned by Weyl in his generalization of geometry. From the viewpoint that the adopted postulates should contain the other theories it then becomes desirable to determine whether or not these postulates specify the geometry of the $\left(\mathrm{dq}^{0}\right)^{2}$ spacetime manifold. More particularly do the adopted postulates lead to a geometry that includes the geometry of current theories? To arrive at a more general geometry would not be a limitation for it would certainly include the others.

Recalling Eq. (19), we can define

$$
\left(d q^{0}\right)^{2}=\left(\frac{1}{f}\right) \hat{\hat{g}}_{i j} d x^{i} d x^{j} \equiv\left(\frac{1}{f}\right)(d \sigma)^{2} \equiv \hat{g}_{i j} d x^{i} d x^{j}
$$

Now the second postulate guarantees the existence of the function mechanical entropy and that $d q^{0}$ be a perfect differential; therefore

$$
d q^{0}=q_{i}^{0} d x^{i},
$$

where $q_{j}^{0} \equiv \partial q^{0} / \partial x^{i}$. Then the exactness of $d q^{0}$ is stated by

$$
q_{i / j}^{0}-q_{j / i}^{0}=0
$$

By defining the parallel displacement of a vector to be

$$
d \zeta_{j}=\hat{\Gamma}_{i s}^{v} d x^{s} \zeta_{v}
$$

and using Eqs. (21) and (22) it may be seen that the connections must be symmetrical, or

$$
\hat{\Gamma}_{i k}^{v}=\hat{\Gamma}_{k i}^{v} .
$$


This result should not be taken to mean that only symmetric connections need to be considered. Rather it means that given the $\hat{g}_{i j}$ 's that maximize $\left(\mathrm{dq}^{0}\right)^{2}=\left(\frac{\overline{\mathrm{d}} E}{\mathrm{~F}_{0} \phi}\right)^{2}$, then the connections are symmetrical. However, since a variational ${ }^{0}$ principle must be used to determine the $\hat{g}_{i j}$ 's, then both symmetric and antisymmetric connections will have to be considered.

In Weyl's generalization of geometry he found it necessary to assume tha symmetry of the connections. He proved a theorem showing that the symmetry of the connections guaranteed the existence of a local Euclidean limiting manifold and used this theorem in support of the symmetry assumption. Here we find that the second law requires that the connections formed by the solution coefficients must be symmetrical thus guaranteeing, through Weyl's theorem, the existence of a local Euclidean gecmetry within the Dynamic Theory.

Suppose now we consider whether the order of differentiating the change in entropy makes any difference. This means that we must use symmetric connections since the actual change in entropy will be determined by the metric coefficients that generate a maximum. Therefore, consider the difference

$$
\Delta\left(d q^{0}\right)^{2} \equiv \frac{\partial^{2}\left(d q^{0}\right)^{2}}{\partial x^{k} \partial x^{l}}-\frac{\partial^{2}\left(d q^{0}\right)^{2}}{\partial x^{l} \partial x^{k}}
$$

Since $\left(d q^{0}\right)^{2}=q_{j}^{0} q_{j}^{0} d x^{i} d x^{j}$ from Eq. (21), using Eq. (20) we find

$$
q_{i}^{0} q_{j}^{0}=\hat{g}_{i j}
$$

Then

$$
\frac{\partial\left(d q^{0}\right)^{2}}{\partial x^{k}}=\left(q_{j \mid k}^{0} q_{i}^{0}+q_{j \mid k}^{0} q_{j}^{0}\right) d x^{i} d x^{j}+\left(q_{k}^{0}\right)^{2} .
$$

Thus

$$
\begin{aligned}
\frac{\partial^{2}\left(d q^{0}\right)^{2}}{\partial x^{k} \partial x^{\ell}}= & {\left[q_{j|k| \ell}^{0} q_{i}^{0}+q_{j \mid k}^{0} q_{i \mid \ell}^{0}+q_{i|k| \ell}^{0} q_{j}^{0}\right.} \\
& \left.+q_{i \mid k}^{0} q_{j \mid \ell}^{0}\right] d x^{i} d x^{j}+2 q_{\ell \mid k}^{0} q_{\ell}^{0}+2 q_{k \mid \ell}^{0} q_{k}^{0} .
\end{aligned}
$$




\section{Likewise}

$$
\begin{aligned}
\frac{\partial^{2}\left(d q^{0}\right)^{2}}{\partial x^{l} \partial x^{k}}= & {\left[q_{j|\ell| k}^{0} q_{i}^{0}+q_{j \mid \ell}^{0} q_{i \mid k}^{0}+q_{i|\ell| k}^{0} q_{j}^{0}+q_{j \mid \ell}^{0} q_{j \mid k}^{0}\right] d x^{i} d x^{j} } \\
& +2 q_{k \mid \ell}^{0} q_{k}^{0}+2 q_{l \mid k}^{0} q^{0} .
\end{aligned}
$$

Therefore the difference must be

$$
\Delta\left(d q^{0}\right)^{2}=\left\{\left(q_{j|k| \ell}^{0}-q_{j|\ell| k}^{0}\right) q_{i}^{0}+\left(q_{i|k| \ell}^{0}-q_{i|\ell| k}^{0}\right) q_{j}^{0}\right\} d x^{i} d x^{j} .
$$

Using the definition Eq. (23) we see that

$$
d q_{i}^{0}=\hat{\Gamma}{ }_{i s}^{r} d x^{s} q_{r}^{0}
$$

and

$$
q_{i \mid k}^{0}=q_{r}^{0} \hat{\Gamma}_{i k}^{r}
$$

also

$$
q_{k / i}^{0}=q_{r}^{0} \hat{\Gamma} \underset{k i}{r}
$$

Now

$$
\begin{aligned}
q_{i|k| l}^{0} & =\frac{\partial}{\partial x^{l}}\left[q_{r}^{0} \hat{\Gamma}_{i k}^{r}\right]=q_{r \mid \ell}^{0} \hat{\Gamma}_{i k}^{r}+q_{r}^{0} \frac{\partial \hat{\Gamma}_{i k}^{r}}{\partial x^{l}} \\
& =q_{s}^{0} \hat{\Gamma}_{r \ell}^{s} \hat{\Gamma}_{i k}^{r}+q_{r}^{0} \frac{\partial \hat{\Gamma}_{i k}^{r}}{\partial x^{l}} \\
& =q_{r}^{0}\left\{\hat{\Gamma}_{i \ell}^{r} \hat{\Gamma}_{i k}^{s}+\frac{\partial \hat{\Gamma} \hat{i k}_{i k}^{r}}{\partial x^{l}}\right\} .
\end{aligned}
$$

\section{Similarly}




$$
q_{i|\ell| k}^{0}=q_{r}^{0}\left\{\hat{\Gamma}_{s k}^{r} \hat{\Gamma}_{i \ell}^{s}+\frac{\partial \hat{\Gamma}_{j}^{r}}{\partial x^{k}}\right\}
$$

Therefore

$$
q_{j}^{0}\left(q_{i|k| \ell}^{0}-q_{i|\ell| k}^{0}\right)=q_{i}^{0} q_{r}^{0}\left\{\frac{\partial \hat{\Gamma}_{i k}^{r}}{\partial x^{\ell}}-\frac{\partial \hat{\Gamma}_{i \ell}^{r}}{\partial x^{k}}+\hat{\Gamma}_{s \ell}^{r} \hat{\Gamma}_{i k}^{s}-\hat{\Gamma}_{s k}^{r} \hat{\Gamma}_{i \ell}^{s}\right\} .
$$

Then defining the vector curvature as

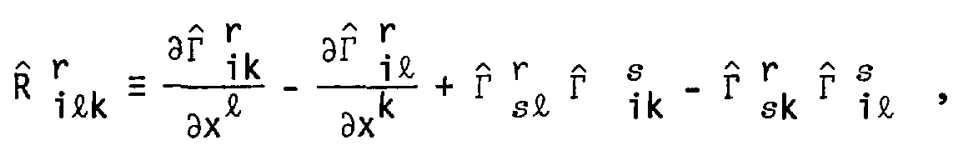

the difference may be written as

$$
\Delta\left(d q^{0}\right)^{2}=\left\{q_{j}^{0} q_{r}^{0} \hat{R}{ }_{i \ell k}^{r}+q_{j}^{0} q_{r}^{0} \hat{R} \underset{j \ell k}{r}\right\} d x^{i} d x^{j} .
$$

However, recall that $q_{i}^{0} q_{j}^{0}=\hat{g}_{i j}$; then

$$
\Delta\left(d q^{0}\right)^{2}=\left\{\hat{g}_{j r} \hat{R}_{i \ell k}^{r}+\hat{g}_{j r} \hat{R}_{j \ell k}^{r}\right\} d x^{i} d x^{j} .
$$

But

$$
\hat{g}_{r i}=\hat{g}_{i r} \text { and } \hat{R}_{i j k \ell}=\hat{g}_{i r} \hat{R}_{j k \ell}^{r} \text {, }
$$

so that

$$
\Delta\left(d q^{0}\right)=\left\{\hat{R}_{j i \ell k}+\hat{R}_{i j \ell k}\right\} d x^{i} d x^{j} .
$$

So the difference will vanish if

$$
\hat{R}_{j i \ell k}=-\hat{R}_{i j \ell k} \text {. }
$$

42 
Now since

$$
\left(d q^{0}\right)^{2}=q_{i}^{0} q_{j}^{0} d x^{i} d x^{j}=\hat{g}_{i j} d x^{i} d x^{j},
$$

differentiation will result in

$$
d\left(d q^{0}\right)^{2}=d\left(q_{j}^{0} q_{j}^{0} d x^{i} d x^{j}\right)=d\left(\hat{g}_{i j} d x^{i} d x^{j}\right)
$$

or

$$
\begin{aligned}
d q_{i}^{0} q_{j}^{0} d x^{i} d x^{j} & +q_{i}^{0} d q_{j}^{0} d x^{i} d x^{j}+q_{i}^{0} q_{j}^{0} d\left(d x^{i} d x^{j}\right) \\
& =d \hat{g}_{i j} d x^{i} d x^{j}+\hat{g}_{i j} d\left(d x^{i} d x^{j}\right),
\end{aligned}
$$

which can be written as

$$
\begin{aligned}
\hat{\Gamma}{ }_{i s}^{r} d x^{s} q_{r}^{0} q_{j}^{0} d x^{i} d x^{j} & +q_{i}^{0} \hat{\Gamma}{ }_{j s}^{r} d x^{s} q_{r}^{0} d x^{i} d x^{j}+q_{i}^{0} q_{j}^{0} d\left(d x^{i} d x^{j}\right) \\
& =d \hat{g}_{i j} d x^{i} d x^{j}+\hat{g}_{i j} d\left(d x^{i} d x^{j}\right) .
\end{aligned}
$$

But

$$
\hat{q}_{i j}=q_{j}^{0} q_{j}^{0} \text {. }
$$

Therefore

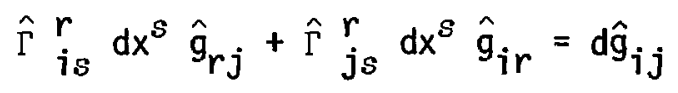

or

$$
\hat{g}_{r j} \hat{\Gamma} r_{i s}^{r}+\hat{g}_{r i} \hat{\Gamma}{ }_{j s}^{r}=\frac{\partial g_{j j}}{\partial x^{s}}
$$

43 
and

$$
\hat{\Gamma}_{j i s}+\hat{\Gamma}_{i j s}=\frac{\partial g_{i j}}{\partial x^{s}}
$$

Now interchange $\mathrm{jis}$ to sij to get

$$
\hat{\Gamma}_{s i j}+\hat{\Gamma}_{j s i}=\frac{\partial g_{j s}}{\partial x^{s}}
$$

Then interchange $j i s$ to $i s j$ so that

$$
\hat{\Gamma}_{i s j}+\hat{\Gamma}_{s i j}=\frac{\partial g_{s i}}{\partial x^{j}}
$$

Add Eqs. (27) and (28) and subtract Eq. (26).

$$
\hat{\Gamma}_{i s j}+\hat{\Gamma}_{s i j}+\hat{\Gamma}_{s i j}+\hat{\Gamma}_{j s i}-\hat{\Gamma}_{j i s}-\hat{\Gamma}_{i j s}=\frac{\partial \hat{g}_{s i}}{\partial x^{j}}+\frac{\partial \hat{g}_{j s}}{\partial x^{i}}-\frac{\partial \hat{g}_{i j}}{\partial x^{s}}
$$

or

$$
\hat{\Gamma}_{s i j}=\frac{1}{2}\left\{\frac{\partial \hat{g}_{s i}}{\partial x^{j}}+\frac{\partial \hat{g}_{s j}}{\partial x^{j}}-\frac{\partial \hat{g}_{i j}}{\partial x^{s}}\right\}
$$

and

$$
\hat{\Gamma}_{i j}^{r}=\hat{g}^{r s} \hat{\Gamma}_{s i j}=\frac{\hat{g}^{r s}}{2}\left\{\frac{\partial \hat{g}_{s i}}{\partial x^{j}}+\frac{\partial \hat{g}_{s j}}{\partial x^{j}}-\frac{\partial \hat{g}_{i j}}{\partial x^{s}}\right\} .
$$

Now by using the symmetry of $\hat{g}_{i j} i t$ can be shown that

$$
\hat{R}_{j i \ell k}=-\hat{R}_{i j \ell k}
$$


and therefore

$$
\Delta\left(\mathrm{dq}^{0}\right)^{2}=0
$$

This is the necessary and sufficient condition that the differential entropy change may be transferred from an initial point to all points of the space in the manner that is independent of the path.

The distinguishing features of Riemannian geometry is the invariance of the scalar product under a vector transplantation. Therefore to determine whether the $\left(\mathrm{dq}^{0}\right)^{2}$ space is a Riemannian space, consider the vector $\hat{\xi}_{j}$ and $\hat{\eta}_{j}$. Now since

$$
\hat{\xi}_{\mathbf{i}}=\hat{g}_{\mathbf{i j}} \hat{\xi}^{\mathbf{j}}
$$

and

$$
d \hat{\xi}_{i}=\hat{\Gamma}{ }_{i s}^{r} d x^{s} \hat{\xi}_{r}=\hat{\Gamma}_{i s}^{r} d x^{s} \hat{g}_{r k} \hat{\xi}^{k}=\frac{\partial g_{i j}}{\partial x^{s}} \hat{\xi}^{j} d x^{s}+\hat{g}_{i j} d \hat{\xi}^{j},
$$

then

$$
\hat{g}_{i j} d \hat{\xi}^{j}=\hat{\Gamma}{ }_{i s}^{r} d x^{s} \hat{g}_{r k} \hat{\xi}^{k}-\frac{\partial g_{i j}}{\partial x^{s}} \hat{\xi}^{j} d x^{s}
$$

Or, since

$$
\hat{g}^{i j} \hat{g}_{i j}=\delta_{i}^{i}=1 \text { and } \frac{\partial \hat{g}_{i j}}{\partial x^{s}}=\hat{\Gamma}_{j i s}+\hat{\Gamma}_{i j s} \text {, }
$$

then 


$$
\begin{aligned}
\mathrm{d} \hat{\xi}^{j} & =\hat{g}^{i j}\left\{\hat{\Gamma}_{k i s} \hat{\xi}^{k}-\left(\hat{\Gamma}_{j i s}+\hat{\Gamma}_{i j s}\right) \hat{\xi}^{j}\right\} d x^{\mathcal{E}} \\
& =\hat{g}^{i j}\left(-\hat{\Gamma}_{i k s}\right) \hat{\xi}^{k} d x^{s} \\
& =-\hat{\Gamma}_{k s}^{j} d x^{s} \hat{\xi}^{k} .
\end{aligned}
$$

Thus the change in the covariant and the contravariant vectors is given by

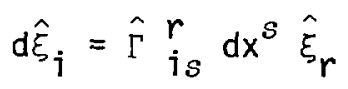

and

$$
\mathrm{d} \hat{\xi}^{\mathbf{i}}=-\hat{\Gamma}_{\mathrm{rs}_{s}}^{\mathbf{i}} \mathrm{dx} \mathrm{x}^{\mathcal{S}} \hat{\xi}^{\mathrm{r}}
$$

Now consider the change in the scalar product $\hat{\xi}_{j} \hat{n}^{i}$. Then

$$
\begin{aligned}
d\left(\hat{\xi}_{i} \hat{\eta}^{i}\right) & =d \hat{\xi}_{i} \hat{\eta}^{i}+\hat{\xi}_{i} d \hat{\eta}^{i} \\
& =\hat{\Gamma}_{i s}^{r} d x^{s} \hat{\xi}_{r} \hat{\eta}^{i}+\hat{\xi}_{i}\left(-\hat{\Gamma}_{r s}^{i} d x^{s} \hat{\eta}^{r}\right) \\
& =\hat{\Gamma}_{i s}^{r} d x^{s} \hat{\xi}_{r} \hat{\eta}^{i}-\hat{\Gamma}_{r s}^{j} d x^{s} \hat{\xi}_{j} \hat{\eta}^{r} .
\end{aligned}
$$

Renaming the indices in the second term yields

$$
d\left(\hat{\xi}_{i} \hat{n}^{i}\right)=\left\langle\hat{I}_{i s}^{r} \hat{\xi}_{r} \hat{n}^{i}-\hat{\Gamma}_{i s}^{r} \hat{\xi}_{r} \hat{n}^{i}\right) d x^{s} .
$$

Thus the geometry of the $\left(d q^{0}\right)^{2}$ manifold is Riemannian.

The next question is what is the geometry of the $(d \sigma)^{2}$ space? Equation (20) shows that we may write $(d \sigma)^{2}=f(d q)^{0}$, which is reminissent of Weyl's 
generalized geometry. Further we have

$$
\hat{\mathrm{g}}_{i j}=f \hat{g}_{i j} \text {. }
$$

Then in the sigma space an arbitrary vector $\xi^{i}$ would have a length $\hat{\hat{\ell}}$ given by the self-scalar product

$$
\hat{\hat{\ell}}^{2} \equiv\|\xi\|^{2}=\hat{\hat{g}}_{i j} \xi^{i} \xi^{j}-f \hat{g}_{i j} \xi^{j} \xi^{j}=f \hat{\ell}^{2},
$$

where $\hat{\ell}$ is the length of the vector in the entropy space.

If we differentiate Eq. (30), we have

$$
2 \hat{\hat{\ell}} \mathrm{d} \hat{\hat{\ell}}=\hat{\ell}^{2} \frac{\partial f}{\partial \mathrm{x}^{i}} \mathrm{dx}+2 \mathrm{f} \hat{\ell} \mathrm{d} \hat{\ell}
$$

However, in the entropy space the length of the vector $\hat{l}$ is unchanged under parallel displacement so that

$$
d \hat{\hat{\ell}}=\frac{1}{2} \frac{\partial f}{\partial x^{i}} d x^{i} \frac{\hat{\ell}}{f}=\frac{1}{2} \frac{\partial \ln f}{\partial x^{i}} d x^{i} \hat{\hat{\ell}} .
$$

Comparing Eq. (31) with the definition of the parallel displacement of a vector, Eq. (23), we find that $\phi_{j} \equiv \frac{\partial \operatorname{lnf}}{\partial x_{i}}$ plays a role similar to that of the

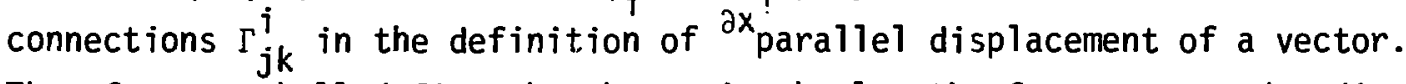

Therefore we shall define the change in the length of a vector under displacement to be

$$
\mathrm{d} \ell=\left(\phi_{j} d x^{i}\right) \ell .
$$

This is the same definition Weyl made in his generalization of geometry. However, there is a difference in the way it was obtained. Heyl chose this definition in analogy with the connections $\Gamma$ and the definition then led to the second more general metric. In this theory the fundamental laws lead us to two metrics and Eq. (31) for the change in the length of a vector under displacement. 
Thus within the Dynamic Theory Eq. (31) is a derived equation and Eq. (32) only renames the logarithmic derivative.

Using Eq. (32) we may obtain, in general,

$$
\begin{aligned}
\mathrm{d} \ell^{2} & =2 \ell^{2}\left(\phi_{i} \mathrm{dx} x^{i}\right)=\mathrm{d}\left(g_{i j} \xi^{i} \xi^{j}\right) \\
& =g_{i j \mid k} \xi^{i} \xi^{j} d x^{k}+g_{i j} \Gamma_{\ell k}^{i} \xi^{\ell} \xi^{j} d x^{k}+g_{i j} \Gamma_{l k}^{j} \xi^{i} \xi^{\ell} d x^{k} .
\end{aligned}
$$

Renaming the various summation indices, rearranging terms, and using the length of a vector, we obtain

$$
\left[g_{i j \mid k}+g_{\ell j} \Gamma_{i j}^{\ell}+g_{i \ell} \Gamma_{j k}^{\ell}\right] \xi^{i} \xi^{j} d x^{k}=2 g_{i j} \phi_{k} \xi^{i} \xi^{j} d x^{k} .
$$

Since this must hold for arbitrary choice of $\xi^{i}$ and $d x^{k}$, we conclude that

$$
\left(g_{i j \mid k}-2 g_{i j} \phi_{k}\right)+g_{\ell j} \Gamma_{i k}^{\ell}+g_{\ell, j} \Gamma_{j k}^{\ell}=0 .
$$

This is the same system of 1 inear equations for the connections $\Gamma_{j k}^{i}$ as Eq. (26) except that the inhomogeneous term $\hat{g}_{i j \mid k}$ has now to be replaced by $g_{i j \mid k}$ $2 g_{i j} \phi_{k}$. Therefore the same linear algebra as before leads to

$$
\Gamma_{j k}^{j}=-\left\{\begin{array}{c}
k \\
j k
\end{array}\right\}+g^{\ell i}\left[g_{\ell j} \phi_{k}+g_{\ell k} \phi_{j}-g_{j k} \phi_{\ell}\right]
$$

where $\left\{\begin{array}{c}i \\ j k\end{array}\right\}$ is the usual Christoffel symbol of the second kind.

Now, since the entropy space is Riemannian, then in the entropy space we have

$$
\hat{\phi}_{\mathbf{i}} \equiv 0 \quad \text { and } \quad \hat{\Gamma}_{j k}^{j}=-\left\{\begin{array}{c}
\mathbf{i} \\
j k
\end{array}\right.
$$

and the length $\hat{\ell}$ of a vector is unchanged under parallel displacement. However, the same displacement law in the sigma space, with metric $\hat{\hat{g}}_{i j}$, leads to the relation 


$$
\begin{aligned}
d \hat{\hat{\ell}} & = \pm d \sqrt{\hat{\hat{g}}_{i j} \xi^{i} \xi^{j}}= \pm d \sqrt{f \hat{g}_{i j} \xi^{i} \xi^{j}} \\
& = \pm \hat{\ell} \frac{\partial \sqrt{f}}{\partial x^{k}} d x^{k} \\
& = \pm \frac{1}{2} \frac{\partial \ell \ln f}{\partial x^{k}} d x^{k} \hat{\hat{\ell}} .
\end{aligned}
$$

Thus $\pm\left(\frac{1}{2}\right)\left(\partial \ln f / \partial x^{k}\right)$ plays the role of $\phi_{k}$ in Eq. (32). It follows then that the ordinary connections $-\left\{\begin{array}{c}\mathbf{j} k \\ \}\end{array}\right\}$ constructed from $\hat{g}_{\mathbf{i j}}$ are equal to the more general connections $\hat{\hat{\Gamma}}_{j k}^{\mathbf{i}}$ constructed according to Eq. (33) from $\hat{\hat{g}}_{i j}$ and $\phi_{k}=\left(\frac{1}{2}\right)\left(\partial \operatorname{lnf} / \partial x^{k}\right)$ :

$$
\hat{\Gamma}_{j k}^{i}=\hat{\Gamma}_{j k}^{i}
$$

This can also be seea by direct computation from Eq. (32) and

$$
\hat{\hat{g}}_{i j}=f \hat{g}_{i j} \text {. }
$$

We may interpret the change of metric from $\hat{g}_{i j}$ to $\hat{\hat{g}}_{i j}$ by Eq. (36) as a change of scale for the length at every point of the Riemannian manifold by the variable gauge factor $f$. This transformation is called a gauge transformation, and $\phi_{k}$ is called a gauge vector field.

The generalized geometry thus separates the problem of measurement of angles from that of measurement of length. For instance, the angle between the two vectors $\xi^{i}$ and $\eta^{i}$ at a given point of the space is measured by the ratio

$$
\frac{\xi^{i} n_{\mathbf{i}}}{\|\xi\|\|n\|}=\frac{g_{i j} \xi^{j} n^{j}}{\left[\left(g_{i j} \xi^{i} \xi^{j}\right)\left(g_{i j} n^{i} n^{j}\right)\right]_{\frac{1}{2}}} .
$$

This ratio does not change under the gauge transformation Eq. (36). The gauge transformation is therefore an angle-preserving, or conformal, change of metric. On the other hand, the length of vectors will change under Eq. (36) according to 
Eq. (31). Thus the metric tensor $\hat{g}_{i j}$ determines angles, while one needs also the gauge vector $\phi_{k}$ to measure length.

Considering the sigma space, which is characterized by the tensor field $\hat{\hat{g}}_{i j}$ and gauge vector $\hat{\phi}_{k}$. The same argument as before shows that we may, without changing the intrinsic geometric properties of vector fields, replace the geometric quantities by use of a scalar field $f$ as follows:

$$
\hat{\hat{g}}_{i j}=f \hat{g}_{i j} \quad \hat{\phi}_{k}=\phi_{k}+\frac{1}{2} \frac{\partial \ln f}{\partial x^{k}} \quad \hat{\hat{\Gamma}}_{j k}^{j}=\hat{\Gamma}_{j k}^{i}
$$

That is, in the new metric, vectors will have the same law of affine transplantation and the angle between different vectors at the same point of the manifold will be preserved, but the local lengths of a vector will be changed according to

$$
\hat{\imath}^{2}=f \hat{\imath}^{2} .
$$

Thus the general Weyl geometry of the sigma space admits also a conformal gauge transformation.

2. Field Equations. With the foregoing, it is apparent that the sigma manifold must have the more general Weyl geometry. Given the vector field $\phi_{k}$ in Eq. (37) we can follow Weyl's interpretations and study the tensor field

$$
F_{i j} \equiv \phi_{i \mid j}-\phi_{j \mid i}
$$

Weyl showed that from a study of this tensor field we are led to the variational principle

$$
\delta \int\left[R+\frac{1}{2} A \tilde{F}_{i j} \tilde{F}^{i j}-\lambda\left(\frac{1}{2}-6 \tilde{\phi}_{i} \tilde{\phi}^{i}\right)\right] \sqrt{-g} d^{4} x=0,
$$

where

$$
\phi_{i} \equiv \sqrt{\lambda} \tilde{\phi}_{i} \text { and } F_{i j} \equiv \sqrt{\lambda} \tilde{F}_{i j} \text {. }
$$


From this variational principle Weyl showed that Einstein's General Relativistic Theory and Maxwell's Electromagnetism may be obtained. The only remaining question here is whether the fector field $\phi_{k}$, which gives rise to the non-vanishing tensor fieid $F_{i j}$, is required by the three fundamental laws adopted by the Dynamic Theory or must be assumed to exist as Weyl did.

If we recall the statement of the first law

$$
\overline{\mathrm{d}} E=\mathrm{d} U-F_{\alpha} \mathrm{dq}{ }^{\alpha}
$$

we find that even for an isolated system there exists the vector field $F_{\alpha}$. Indeed, in Section II.C.1 it was shown that this vector field is inevitable. Further recall that the geometry given by the stability conditions is based upon the second partial derivatives of the system's energy function, $U$, and does not include the forces $F_{\alpha}$. It seems only logical to suspect that there exists a link between the vector field $\phi_{i}$ and the forces $F_{\alpha}$, especially since the work terms àre path dependent and the nonvanishing of the curl components of $\phi_{i}$ is equivalent to the statement that the potential function $\phi$ is path dependent.

The connection between the potential vector field $\phi_{j}$ and the forces $F_{\alpha}$ comes from Maxwell's electromagnetic theory where the forces $F_{\alpha}$ are the Lorentz forces made up of the components $\phi_{i}$. However, we shall not show the connection here since any teit on Weyl's theory, or electromagnetism, displays the connection. We sha 11, however, present it during the development of the more general five-dimensional system. For the present though, if we adopt Weyl's interpretation plus taking the forces $F_{\alpha}$ to be the forces resulting from the $\phi_{i}$, we then may see that the Dynamic Theory leads us, through Weyl's unified theory, to Einstein's General Relativistic and Maxwell's Electromagnetic theories and further, as special cases, the Special Relativistic Theory and Newtonian mechanics.

\section{E. Mechanical Systems Near Equilibrium}

We have shown how, for an isolated system subjected to only three spatial forces, the Dynamic Theory includes Einstein's relativistic and Maxwell's electromagnetic theories. However the approach has been abstract and does not readily display the effect of the new point of view upon classical physical concepts. It then seems beneficial to pause in the development and take a different look at what the Dynamic Theory says about mechanics. 
1. Classical Mechanics. Classical mechanics describes the motion of a system, which could be a particle, for which the energy of the system is a constant. The equations of motion may be obtained using Hamilton's principle. These equations of motion yield trajectories resulting from the action of forces; they may also be obtained from the principle of least action. When the action integral is treated as a variational problem with variable end points, the method of Lagrangian multipliers yields the same equations as does Hamilton's principle. However, if the variational problem is transformed to a new space in which the new variational problem has fixed end points, then the metric for this space is displayed, and the equations of motion are geodesics in this space.

In classical mechanics the principle of least action as formulated by Lagrange has the integral form

$$
A=\int_{P_{1}}^{P_{2}} m \bar{v} \cdot d \bar{s} .
$$

In curvilinear coordinates the integral assumes the form

$$
A=\int_{P_{1}}^{P_{2}} m g_{\alpha \beta} \frac{d x^{\alpha}}{d t} d x^{\beta}=\int_{t\left(p_{1}\right)}^{t\left(p_{2}\right)} m g_{\alpha \beta} \frac{d x^{\alpha}}{d t} \frac{d x^{\beta}}{d t} d t,
$$

where $\alpha, \beta=1,2,3$.

Defining

$$
T \equiv \frac{m}{2} g_{\alpha \beta} \frac{d x^{\alpha}}{d t} \frac{d x^{\beta}}{d t}
$$

the integral becomes

$$
A=\int_{t\left(p_{1}\right)}^{t\left(p_{2}\right)} 2 T d t
$$


Then the principle of least action may be stated as:

of all curves $C^{\prime}$ passing through $P$ and $P$ in the neighborhood of the trajectory $C$, which are traversed at a rate such that, for each $C^{\prime}$, for every value of $t, T+V=F$, that one for which the action integral $A$ is stationary is the trajectory of the particle.

The transformation of variables may be carried out to display the metric

$$
(d s)^{2}=h_{\alpha \beta} d x^{\alpha} d x^{\beta}
$$

where

$$
h_{\alpha \beta}=2 m\left(\varepsilon_{0}-V\right) g_{\alpha \beta} .
$$

Here different particles in the same field and with different energies $\varepsilon_{0}$ would appear to have different geometries, a situation which has been previously taken to be impossible and therefore precluded the geometrization of dynamics (see page 6 of reference 1). However, in view of Weyl's generalization of geometry, treating the variational problem in the principle of least action as transformed to a new space in which the variational problem has fixed end points, in effect, is a transformation into a space with Weyl geometry where the gauge function is $2 m\left(\varepsilon_{0}-v\right) g_{\alpha \beta}$. Thus changing the energies $\varepsilon_{0}$ does not change the geometry since it will still be a Weyl space.

Suppose now that the concepts of classical mechanics are compared with the concepts from the point of view of the Dynamic Theory. The energy of the system in classical mechanics is a constant of the motion and therefore the change in kinetic energy is the negative of the change in potential energy, which may be written as

$$
\mathrm{dH}=\mathrm{dT}+\mathrm{dV}=0 .
$$

However, for classically conservative forces $\mathrm{dH}$ is a perfect differential. Therefore for this system with only one work term the force is a function of position only. 
This suggests the association of the classical energy of the system, $\mathrm{H}$, with the system energy, $U$, which is also a perfect differential. Now if the system is isolated, or $E$-conservative, then

$$
0=\overline{\mathrm{d}} E=\mathrm{d} U-F \mathrm{dq}
$$

But $t$ if $\mathrm{d} U=\mathrm{d} H=0$ then $F$ must be zero. This points out an important difference between classical physics and the Dynamic Theory. A classically conservative system is one for which the system's energy is a constant of the motion. However, the E-conservative system, within the Dynamic Theory, is one for which $\overline{\mathrm{d}} E=0$. Thus an $E$-conservative sy'stem which is also conservative in the classical sense must have no forces $F$ which nay depend upon velocity as well as position but may have forces which arise from $-\frac{\partial U}{\partial q}=F$ and must be functions of positions orily.

2. Relativistic Mechanics. Suppose we now turn our attention to the mechanics of special relativity. In the special theory of relativity Einstein sought to put Newtonian mechanics into a form which would leave the speed of light invariant. The resulting dynamics exhibits the notion of a unique velocity in a similar sense to the previously defined absolute velocity. The modification required the motion to be such that

$$
\int_{t_{1}}^{t_{2}}\left(\frac{m \dot{q} \dot{q}}{\sqrt{1-\dot{q}^{2} / c^{2}}}+F \delta q\right) d t=0 \text {, }
$$

where $F$ is a force which is a function of position only.

The factor $\sqrt{1-\dot{q}^{2} / c^{2}}$ displays the qualities required of the integrating factor $\phi(\dot{q})$. Therefore consider a modification of Hamilton's principle in terms of the system energy $U$, the force $F$, and the integrating factor $\phi$. The modified statement then would be that the motion be such that

$$
\int_{t_{1}}^{t_{2}}\left(\frac{\delta U}{\phi}+\frac{F}{\phi} \delta q\right) d t=0 .
$$

It can be seen that if $\phi(\dot{q})=1$, then $F$ must be a function of only $q$ and classical mechanics results. It will be shown that if $\phi(\dot{q})=\sqrt{1-\dot{q}^{2} / c^{2}}$ relativistic 
mechanics is obtained.

Now for an isentropic system

$$
\mathrm{d} S=\frac{\overline{\mathrm{d}} E}{\phi}=0=\frac{\mathrm{d} U}{\phi}-\frac{F}{\phi} \mathrm{dq}
$$

or

$$
\int_{A}^{\mathrm{B}} \frac{\mathrm{d} U}{\phi}=\int_{\dot{A}}^{\mathrm{B}} \frac{F}{\phi} \mathrm{dq} .
$$

This would be the classical work-energy theorem if $\phi=1$. For any $\phi$

$$
\frac{\mathrm{d} U}{\mathrm{~d} t}=F \dot{\mathrm{q}}
$$

If the system energy $U$ is taken to be the kinetic energy and defined as

$$
U \equiv \frac{1}{2} m \dot{q}^{2},
$$

then

$$
m \ddot{q}=F,
$$

or Newton's second law.

This tends to indicate that a modification of Hamilton's principle would apply to a system for which $\mathrm{d} S=0$. This modification would be to assume that for an isentropic system the motion is given by the principle:

If a particle is at the point $P_{1}$ at the time $t_{1}$ and at the point $P_{2}$ at the time $t_{2}$, then the motion of the particle takes pTace in such a way that

$$
\int_{t_{1}}^{t_{2}}\left(\frac{\delta U}{\phi(\hat{q})}+\frac{F}{\phi(\hat{q})} \delta q\right) d t \equiv \delta \int_{t_{1}}^{t_{2}} L d t=0 \text {, }
$$


where $q=q(t)$ is the generalized coordinate of the particle along the trajectory and $q+\delta q$ is the coordinate along a varied path beginning at $P_{1}$ at the time $t_{1}$ and ending at $P_{2}$ at time $t_{2}$.

The hypothesis of the fundamental lemma of calculus of variations is that $L$ be a real continuous function, therefore, the mixed second partial derivatives of $L$ must be equal, or

$$
\frac{\partial^{2} L}{\partial \dot{q} \partial q}=\frac{\partial^{2} L}{\partial q \partial \dot{q}}
$$

Now

$$
\mathrm{d} L=\frac{1}{\phi(\dot{\mathrm{q}})} \frac{\partial U}{\partial \dot{\mathrm{q}}} \mathrm{d} \dot{\mathrm{q}}+\frac{1}{\phi(\dot{\mathrm{q}})}\left[\frac{\partial U}{\partial \mathrm{q}}+F\right] \mathrm{dq},
$$

so that

$$
\frac{\partial L}{\partial \dot{q}}=\frac{1}{\phi} \frac{\partial U}{\partial \dot{q}} \quad \text { and } \quad \frac{\partial L}{\partial q}=\frac{1}{\phi}\left[\frac{\partial U}{\partial q}+F\right] \text {. }
$$

Then

$$
\frac{\partial^{2} L}{\partial q \partial \dot{q}}=\frac{1}{\phi} \frac{\partial^{2} U}{\partial q \partial \dot{q}}=\frac{1}{\phi}\left(\frac{\partial^{2} U}{\partial \dot{q} \partial q}+\frac{\partial F}{\partial \dot{q}}\right)-\frac{\phi^{\prime}}{\phi^{2}}\left[\frac{\partial U}{\partial q}+F\right] \text {. }
$$

This requires that

$$
\frac{\partial F}{\partial \dot{q}}=\frac{\phi^{\prime}}{\phi}\left(\frac{\partial U}{\partial q}+F\right)
$$

However, $\mathrm{d} S$ is a perfect differential so that

$$
\frac{\partial^{2} S}{\partial q \partial \dot{q}}=\frac{\partial^{2} S}{\partial \dot{q} \partial q}
$$

56 
Since

$$
d S=\frac{1}{\phi} \frac{\partial U}{\partial \dot{q}} d \dot{q}+\frac{1}{\phi}\left(\frac{\partial U}{\partial q}-F\right) d q
$$

and

$$
\frac{1}{\phi} \frac{\partial^{2} U}{\partial q \partial \dot{q}}=\frac{1}{\phi}\left(\frac{\partial^{2} U}{\partial \dot{q} \partial q}-\frac{\partial F}{\partial \dot{q}}\right)-\frac{\phi^{\prime}}{\phi}\left(\frac{\partial U}{\partial q}-F\right),
$$

or

$$
\frac{\partial F}{\partial q}=-\frac{\phi^{\prime}}{\phi}\left(\frac{\partial U}{\partial q}-F\right)
$$

In order that $d S$ and $d L$ both be perfect differentials at the same time then

$$
\frac{\partial F}{\partial \dot{q}}=-\frac{\phi^{\prime}}{\phi}\left(\frac{\partial U}{\partial q}-F\right)=\frac{\phi^{\prime}}{\phi}\left(\frac{\partial U}{\partial q}+F\right) \text {. }
$$

\section{Therefore}

$$
\frac{\partial U}{\partial q}=0
$$

and

$$
\frac{\partial F}{\partial \vec{q}}=\frac{\phi^{\prime}}{\phi} F
$$

or

$$
\frac{1}{F} \frac{\partial F}{\partial \dot{q}}=\frac{\phi^{\prime}}{\phi},
$$


which is a function of $\dot{q}$ only. Then

$$
\frac{1}{\phi} \frac{\partial F}{\partial \dot{q}}-\frac{\phi^{\prime}}{\phi^{2}} F=0
$$

or

$$
\frac{\partial}{\partial \dot{q}}\left(\frac{F}{\phi}\right)=0
$$

which implies that

$$
\frac{F}{\phi}=F(q)
$$

which is a function of $q$ only, and

$$
F=\phi(\dot{q}) \quad F(q) .
$$

Suppose that the momentum is defined as

$$
p \equiv \frac{\partial L}{\partial \dot{q}}=\frac{1}{\phi} \frac{\partial U}{\partial \dot{q}}
$$

and the mass is defined as

$$
m=\frac{\partial^{2} U}{\partial \dot{q}^{2}}
$$

Then

$$
d S=p d \dot{q}-F(q) d q,
$$

and 
$d L=p d \dot{q}+F(q) d q$.

The equations of motion would be

$$
\frac{\mathrm{d}}{\mathrm{dt}}\left[\frac{\partial L}{\partial \dot{q}}\right]-\frac{\partial L}{\partial \mathbf{q}}=0,
$$

or

$$
\begin{aligned}
& \frac{d p}{d t}=\frac{\partial L}{\partial q}=F(q) \\
& \text { If } m=\text { constant, then } p=m \dot{q} / \phi \text { and } \\
& d L=\frac{m \dot{q}}{\phi} d \dot{q}+F(q) d q
\end{aligned}
$$

while

$$
\mathrm{d} S=\frac{\mathrm{m} \dot{q}}{\phi} \mathrm{dq}-\mathrm{F}(\mathrm{q}) \mathrm{dq}
$$

Then for $d S=0$,

$$
S=\tau(\dot{q})+V(q) \quad \text { and } \quad L=\tau(\dot{q})-V(q) \text {, }
$$

where

$$
F(q)=-\frac{\partial V(q)}{\partial q}
$$

How then may $\phi(\dot{q})$ be determined? The precedence set by thermodynamics is to determine $\phi(\dot{q})$ experimentally. Experiments with a charged particle in a magnetic field, such as a mass spectrometer, show that

$$
\phi(\dot{q})=\sqrt{1-\dot{q}^{2} / c^{2}} .
$$


In special relativity this factor comes from the metric of Minkowski's spacetime manifold. The Dynamic Theory gives us the same factor for systems which are very near an equilibrium state since for such a system the coefficients of the metric become constants, which are the second partial derivatives of the system energy function evaluated at the equilibrium state. Therefore the Dynamic Theory produces Einstein's Special Theory of Relativity for an isolated system very near an equilibrium state.

Notice that the integrating factor in Eq. (45) satisfies the requirements for the integrating factor with $c$ as the absolute velocity.

If this integrating factor is substituted into the equations of motion, the resulting equation is

$$
\frac{d}{d t}\left[\frac{m \dot{q}}{\sqrt{1-\dot{q}^{2} / c^{2}}}\right]=F(q) \text {. }
$$

Then

$$
\mathrm{d} L=\frac{\mathrm{m} \dot{\mathrm{q}} \dot{\mathrm{q}}}{\sqrt{1-\dot{q}^{2} / \mathrm{c}^{2}}}+\mathrm{F}(\mathrm{q}) \mathrm{dq}
$$

and

$$
\begin{aligned}
L(\dot{q}, q)-L\left(\dot{q}_{0}, q_{0}\right) & =\int_{\dot{q}_{0}}^{\dot{q}} \frac{m \dot{q} d \dot{q}}{\sqrt{1-\dot{q}^{2} / c^{2}}}+\int_{q_{0}}^{q} F(q) d q \\
& =\left.m c^{2} \sqrt{1-\dot{q}^{2} / c^{2}}\right|_{\dot{q}_{0}} ^{\dot{q}}-v(q)+v\left(q_{0}\right) .
\end{aligned}
$$

If $L\left(\dot{q}_{0}, q_{0}\right)=L(0, \infty)=0$, then

$$
L(\dot{q}, q)=m c^{2}\left[1-\sqrt{1-\dot{q}^{2} / c^{2}}\right]-v(q) .
$$


With the exception of the additive term $\mathrm{mc}^{2}$ this is the form of the relativistic Lagrangian when $m$ is interpreted as the rest mass, and since additive constants in the Lagrangian do not affect the equations of motion, this Lagrangian yields equations of motion consistent with the special theory of relativity.

The first integral of the equations of motion may be written as

$$
\frac{\mathrm{d}}{\mathrm{dt}}\left[L-\dot{\mathrm{q}} \frac{\partial L}{\partial \dot{\mathrm{q}}}\right]=\frac{\mathrm{d}}{\mathrm{dt}}[L-\dot{\mathrm{q} p}]=0,
$$

therefore

$$
L-\dot{q} p=\text { constant } \text {. }
$$

Then define this constant of the motion, which may be called a "Hamiltonian," by

$$
H \equiv \dot{q} \mathrm{p}-L
$$

Since the Lagrangian is giver, by

$$
L=\int p d \dot{q}-V(q),
$$

the Hamil tonian becomes

$$
H=\dot{q} p-\int p d \dot{q}+V(q)=\int \dot{q} d p+V(q)
$$

Then the Hamiltonian equations of motion may be written as

$$
\frac{\partial H}{\partial q}=-F(q)=-\frac{d p}{d t} ; \frac{\partial H}{\partial p}=\dot{q} .
$$

For the particular Lagrangian

$$
L=m c^{2}\left[1-\sqrt{1-\dot{q}^{2} / c^{2}}\right]-v(q),
$$


the Hamiltonian is

$$
\begin{aligned}
H & =\frac{m \dot{q}}{\sqrt{1-\dot{q}^{2} / c^{2}}}-m c^{2}\left[1-\sqrt{1-\dot{q}^{2} / c^{2}}\right]+V(q) \\
& =m c^{2}\left(\frac{1}{\sqrt{1-\dot{q}^{2} / c^{2}}}-1\right)+V(q)
\end{aligned}
$$

or

$$
H=m c^{2}(\gamma-1)+V(q),
$$

where

$$
\gamma \equiv \frac{1}{\sqrt{1-\dot{q}^{2} / c^{2}}}
$$

Then defining $E(\dot{q})-E_{0} \equiv m c^{2}(\gamma-1)$ implies that

$$
E^{2}=E_{0}^{2}+(p c)^{2} \text {. }
$$

In the special theory of relativity the Hamiltonian, which is interpreted as the energy of the system when $m$ is the rest mass and $c$ is the speed of light, has the same form as Eq. (53). However, in the Dynamic Theory the Hamiltonian is not the energy of the system since it is the system energy $U$ and is here given by $U=\frac{1}{2} m \dot{q}^{2}$ since $\frac{\partial U}{\partial q}$ was taken to be zero.

Thus interrelationships between mechanical concepts of classical mechanics, relativistic mechanics, and the Dynamic Theory may be displayed. This approach does not show the rigorous development which could have been achieved by beginning with the metric, restricting the system to be very near an equilibrium state in order to obtain constant coefficients, and then applying the principle of increasing mechanical entropy. Such a procedure only duplicates Einstein's development of the special theory from Minkowski's space-time continuum and is also presented in a generalized version later in this report. Therefore the rigorous development will not be done here. 
Suppose we now return to the question of whether the force is really a function of position only or is also a function of velocity as taken to be the case in the introduction. To view this consider the differential expression of the first law

$$
\overline{\mathrm{d}} E=\mathrm{d} U-F \mathrm{dq} .
$$

But $U=\frac{1}{2} m \dot{q}^{2}$ and $F=\phi F(q)=\sqrt{1-\dot{q}^{2} / c^{2}} F(q)$, so that

$$
\overline{\mathrm{d}} E=m \dot{q} d \dot{q}-\sqrt{1-\dot{q}^{2} / c^{2}} F(q) d q .
$$

If the first law is taken to be the "real" expression of conservation of energy, as is the case in the Dynamic Theory, then the "real force," $F$, displays the velocity dependence believed to be the case. On the other hand the mass appearing in the first law is the "rest" mass and does not depend upon the velocity. However, the first law is a path dependent function and as such may not be integrated until the path is known; therefore its utility is 1 imited.

The second law provides a path independent function whose differential is given by

$$
\mathrm{d} S=\frac{\mathrm{d} U}{\ddot{\phi}}-\frac{F}{\phi} \mathrm{dq}
$$

or, since $U=\frac{1}{2} m \dot{q}^{2}, F=\sqrt{1-\dot{q}^{2} / c^{2}} F(q)$, and $\phi=\sqrt{1-\dot{q}^{2} / c^{2}}$,

$$
d S=\frac{m \dot{q}}{\sqrt{1-\dot{q}^{2} / c^{2}}} d \dot{q}-F(q) d q \quad .
$$

In this differential we see the appearance of the relativistic, or "effective," mass and an "effective" force which is a function of position only. Thus it is obvious that we cannot interchangeably use the concept of rest mass and velocity dependent forces as compared to relativistic mass and velocity independent forces. This is because, in the Dynamic Theory, there are two differential expressions which may not be used interchangeably since one is the result of the 
first fundamental law and is not an exact differential while the other comes as the result of the second fundamental law and is exact.

3. Non-Isolated System. Thus far we have consistently required the system to be isolated. Obviously there are a large number of physical phenomena for which this restriction may not be used, even as an approximation. Therefcre, relaxation of this restriction should provide description of a large and important class of systems.

One of the benefits of the Dynamic Theory is the capability of using procedures currently used in one branch of physics in another where prior to the unification displayed here would have been thought impossible. A system in which this procedure should produce significant results is a nonequilibrium thermodynamic system. Thermodynamics tells us that we must minimize the free energy, but the ability to use this as a variational principle to obtain equations of motion is a procedure which the Dynamic Theory now makes possible for this thermodynamic system.

This research has not yet considered nonisolated systems though some discussion of this class of systems is contained in reference (9); they remain a possible subject for future research.

F. Quantum Effects

Before displaying the quantum effects of the Dynamic Theory it seems important to briefly discuss philosophy with regard to current quantum theory. The quantum theoretical structure has swept aside virtually every attack upon it. Indeed its successes have mounted so rapidly and stand so firmly that to express disagreement is taken as sacrilegious. Yet it seems difficult to subscribe to a11 the teachings of current quantum physics. However, this skepticism concerns only the implication that Heisenberg's uncertainty principle applies to all physical phenomena.

On the other hand it does not seem possible either to embrace completely Einstein's celebrated quote "God does not play with dice." Rather, it should be modified to read "God does not play with dice all the time." Should not quantum effects depend upon the constraints placed upon a given system? This is akin to a vibrating string that is anchored at both ends and vibrates, ideally, in certain allowed modes or a string that vibrates at one end with the other end free.

How then can the limits of applicability of the uncertainty principle be obtained if indeed there are any imits to be placed upon it? Suppose we 
consider the Poisson brackets, which are fundamental in classical mechanics, and form the link between classical physics and quantum physics through the correspondence principle.

The classical Poisson brackets represent the Euler equations resulting from a variational problem minimizing a functional subject to a constraint. Therefore consider the definition, in tensor formalism,

$$
[f, g] \equiv \frac{\partial f}{\partial x^{i}} \frac{\partial g}{\partial \tilde{p}_{i}} \frac{\partial f}{\partial \tilde{p}_{i}} \frac{\partial g}{\partial x^{i}}
$$

where the canonical momenta $\tilde{p}_{j}$ is defined in the classical fashion. Let $f=x^{i}$ and $\mathbf{g}=\mathrm{H}$, where $\mathrm{H}$ is the Hamiltonian, then

$$
\begin{aligned}
{\left[x^{j}, H\right] } & =\frac{\partial x^{j}}{\partial x^{i}} \frac{\partial H}{\partial \tilde{p}_{i}}-\frac{\partial x^{j}}{\partial \tilde{p}_{i}} \frac{\partial H}{\partial x^{i}} \\
& =\frac{\partial H}{\partial \tilde{p}_{j}}+\frac{\partial x^{j}}{\partial \tilde{p}_{i}} \frac{d \tilde{p}_{i}}{d t},
\end{aligned}
$$

since the equations of motion yield

$$
\dot{x}^{i}=\frac{d x^{i}}{d t}=\frac{\partial H}{\partial \tilde{p}_{i}} \quad \text { and } \quad-\tilde{\dot{p}}_{i} \equiv-\frac{d \tilde{p}_{i}}{d t}=\frac{\partial H}{\partial x^{\dot{j}}} \text {. }
$$

Therefore

$$
\left[x^{j}, H\right]=\dot{x}^{j}
$$

if $x^{j}$ is independent of the $\tilde{p}_{j}^{\prime}$ 's so that

$$
\frac{\partial x^{j}}{\partial \tilde{p}_{i}}=0
$$

Consider 


$$
\begin{aligned}
{\left[\tilde{p}_{j}, H\right] } & =\frac{\partial \tilde{p}_{j}}{\partial x^{j}} \frac{\partial H}{\partial \tilde{p}_{i}}-\frac{\partial \tilde{p}_{j}}{\partial \tilde{p}_{i}} \frac{\partial H}{\partial x^{\dot{j}}} \\
& =\frac{\partial \tilde{p}_{j}}{\partial x^{j}} \dot{x}^{i}+\delta_{i j} \frac{d p_{i}}{d t} .
\end{aligned}
$$

Then $\left[\hat{p}_{1 j} H\right]=\frac{d \tilde{p}_{j}}{d t}$

if $\tilde{p}_{j}$ is independent of the $x^{j}$ 's so that $\frac{\partial \tilde{p}_{j}}{\partial x^{i}}=0$.

Thus if the $\tilde{\mathrm{p}}_{j}$ 's and the $x^{i}$ 's are independent, then Eqs. (55) and (56) are the Poisson bracket equations of motion.

Now consider the fundamental Poisson brackets

$$
\left[x^{i}, x^{j}\right],\left[\tilde{p}_{j}, \tilde{p}_{j}\right] \text {, and }\left[x^{i}, \tilde{p}_{j}\right] \text {. }
$$

First

$$
\begin{aligned}
{\left[x^{i}, x^{j}\right] } & =\frac{\partial x^{i}}{\partial x^{k}} \frac{\partial x^{j}}{\partial \tilde{p}_{k}}-\frac{\partial x^{i}}{\partial \tilde{p}_{k}} \frac{\partial x^{j}}{\partial x^{k}} \\
& =\delta_{i k} \frac{\partial x^{j}}{\partial \tilde{p}_{k}}-\delta_{j k} \frac{\partial x^{i}}{\partial \tilde{p}_{k}},
\end{aligned}
$$

which is

$$
\left[x^{i}, x^{j}\right]=\frac{\partial x^{j}}{\partial \tilde{p}_{i}}-\frac{\partial x^{i}}{\partial \tilde{p}_{j}}
$$

or $\left[x^{i}, x^{j}\right]=0$ if (1) the $x^{i}$ s are independent of the $\tilde{p}_{i}$ 's or (2) if $\frac{\partial x^{j}}{\partial \tilde{p}_{j}}=\frac{\partial x^{j}}{\partial \tilde{p}_{j}}$. 
The next fundamental bracket is

$$
\left[\tilde{p}_{i}, \tilde{p}_{j}\right]=\frac{\partial \tilde{p}_{i}}{\partial x^{k}} \frac{\partial \tilde{p}_{j}}{\partial \tilde{p}_{k}}-\frac{\partial \tilde{p}_{i}}{\partial \tilde{p}_{k}} \frac{\partial^{\tilde{p}_{j}}}{\partial x^{k}}
$$

or $\left[\tilde{p}_{i}, \tilde{p}_{j}\right]=0$ if (1) the $P_{i}^{\prime} s$ are independent of the $x^{i}$ 's or (2) if $\frac{\partial \tilde{p}_{j}}{\partial_{x}^{j}}=\frac{\partial \tilde{p}_{j}}{\partial_{x^{j}}}$. The third bracket is

$$
\begin{aligned}
{\left[x^{i}, \tilde{p}_{j}\right] } & =\frac{\partial x^{i}}{\partial x^{k}} \frac{\partial \tilde{p}_{j}}{\partial \tilde{p}_{k}}-\frac{\partial x^{i}}{\partial \tilde{p}_{k}} \frac{\partial \tilde{p}_{j}}{\partial x^{k}} \\
& =\delta_{i k} \delta_{j k}-\frac{\partial x^{j}}{\partial \tilde{p}_{k}} \frac{\partial \tilde{p}_{j}}{\partial x^{k}}
\end{aligned}
$$

or $\left[x^{j}, p_{j}\right]=\delta_{i j}-\frac{\partial x^{i}}{\partial \tilde{p}_{k}} \frac{\partial^{\tilde{p}}}{\partial x^{k}}$

Then

$$
\left[x^{i}, \tilde{p}_{j}\right]=\delta_{i j}
$$

if the $\tilde{p}_{j}^{\prime} s$ and $x^{j}$ 's are independent.

Thus it can be seen that if the coordinates and canonical momenta are independent, then the classical Poisson brackets are given by

$$
\begin{aligned}
& {\left[x^{i}, x^{j}\right]=0,} \\
& {\left[\tilde{p}_{j}, \tilde{p}_{j}\right]=0, \text { and }} \\
& {\left[x^{i}, \tilde{p}_{j}\right]=\delta_{i j} .}
\end{aligned}
$$

Indeed this is the condition used in developing the Lagrange and Poisson brackets. However, the tensor formalism may be used to investigate the requirements 
placed upon the metric coefficients for this condition of independence of momenta and coordinates to hold.

$$
\begin{aligned}
& \text { Consider }\left[x^{i}, \tilde{p}_{j}\right] \text { if the condition } \frac{\partial \tilde{p}_{j}}{\partial x^{j}}=\frac{\partial \tilde{p}_{j}}{\partial x^{i}} \text { ho]ds. For this condition } \\
& \begin{aligned}
{\left[x^{i}, \tilde{p}_{j}\right] } & =\delta_{i j}-\frac{\partial x^{i}}{\partial \tilde{p}_{k}} \frac{\partial \tilde{p}_{j}}{\partial x^{k}}=\delta_{i j}-\frac{\partial x^{j}}{\partial \tilde{p}_{k}} \frac{\partial \tilde{p}_{k}}{\partial x^{j}} \\
& =\delta_{i j}-\frac{\partial x^{j}}{\partial x^{j}}=\delta_{i j}-\delta_{i j}
\end{aligned}
\end{aligned}
$$

or

$$
\left[x^{i}, \tilde{p}_{j}\right]=0
$$

Therefore if $\frac{\partial \tilde{p}_{i}}{\partial x^{j}}=\frac{\partial \tilde{p}_{j}}{\partial x^{i}}$, then $x^{i}$ commutes with $\tilde{p}_{j}$. What conditions must the $g_{i j}$ 's in the metric for the space satisfy in order to achieve this condition? The canonical momenta are given by

$$
\tilde{p}_{j}=m g_{i j} \dot{x}^{j}
$$

where in general the $g_{i j}$ 's are runctions of the $x^{i}$ 's. Hence the condition required for the momenta to be independent of the coordinates is that the $g_{i j}$ 's be constants. For this condition to be met it is necessary and sufficient for the Riemann curvature tensor formed from the $g_{i j}$ 's to be identically zero. This requires that the Gaussian and Einstein curvatures to be zero also since the Riemann tensor is

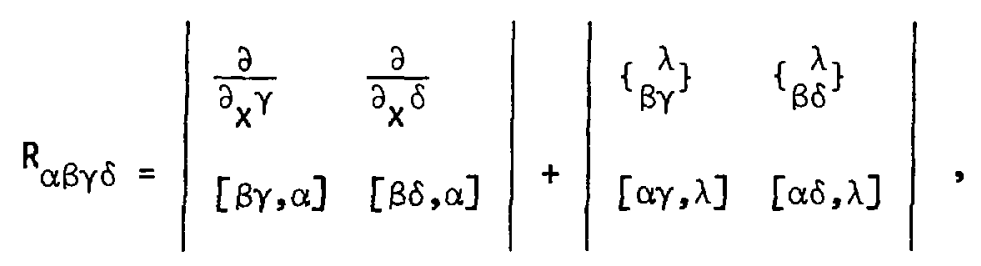


the Einstein curvature is

$$
R=g^{\mu \nu} R_{\mu \nu}=g^{\lambda \alpha} g^{\mu \nu} R_{\lambda \mu \nu \alpha},
$$

and the Gaussian curvature is

$$
K=-\frac{1}{2} \mathbf{R}
$$

The vanishing of the curvature is necessary and sufficient for the existence of a transformation to a coordinate system where the metric coefficients are constants and therefore guarantees that the canonical transformation in classical mechanics can be made. Then the conditions for

$$
\left[x^{i}, \tilde{p}_{j}\right]=\delta_{i j}
$$

is the vanishing of the space curvature.

The equation $R_{i j}=\rho g_{i j}$ is Einstein's gravitational equation at points where matter is present. Therefore, when the Riemann curvature tersor vanishes, the rectilinear geodesics of the manifolds corresponds to the trajectories of particles in the absence of a gravitational field. Consequently, if the manifold with the quadratic form

$$
(d s)^{2}=g_{i j} d x^{i} d x^{j}
$$

is to account for nonrectilinear trajectories, the Riemann curvature tensor must not vanish. Then the $\tilde{p}_{j}$ and the $x^{i}$ will not be independent and the fundamental Poisson brackets cannot be written as given by Eq. (57).

${ }_{\partial} \tilde{p}_{j}$ In particular, the existerice of a function $\rho$ in $R_{i j}=\rho g_{i j}$ such that $\frac{\partial \tilde{p}_{i}}{\partial_{x}}=$ $\frac{\partial^{p} j}{\partial x^{j}}$ is possible and in that event $\left[x^{i}, \tilde{p}_{j}\right]=0$. Now the quantum Poisson brackets are required, through the correspondence principle, to correspond to the classical Poisson brackets. Obviously in the event of a curved space it is possible that the $x^{i}$ 's and $\tilde{p}_{i}$ 's commute thus removing support for the applicability of Heisenberg's uncertainty principle. This, of course, cannot be taken as proof that the uncertainty principle is applicable only in Euclidean spaces since the 
correspondence principle is a correspondence only. However, it does represent a possible limit of applicability which we shall see again from a different point of view.

In $1927 \mathrm{~F}$. London derived quantum principles from Weyl's geometry. However, the results of his work made it difficult to define length as a real number and because of this Weyl later interpreted the mathematical formalism of his unified theory as connected with transplanting a state vector of a quantum-theoretical system.

Suppose that we consider an isolated, or $E$-conservative, system so that $\overline{\mathrm{d}} E$ : 0 . Then, because of the second $1 \mathrm{aw} \mathrm{dq} \mathrm{u}^{\mathrm{U}} \geq 0$ which is the principle of increasing mechanical entropy. Then certainly $\left(\mathrm{dq}^{0}\right) \geq 0$ and also, since

$$
\left(d q^{0}\right)^{2}=f(d \sigma)^{2}
$$

then

$$
f(d \sigma)^{2} \geq 0
$$

However, if $\mathbf{f}<0$, then $(\mathrm{d} \sigma)^{2}<0$ since it is the product that must remain greater than, or equal to, zero. In this case

$$
d q^{0}=\sqrt{-f} \sqrt{-(d \sigma)^{2}}
$$

But

$$
d(d \sigma)=\phi_{k} d x^{k}(d \sigma)
$$

and

$$
\int \frac{d(d \sigma)}{(d \sigma)}=\int \phi_{k} d x^{k},
$$

which implies that the element of arc $(d \sigma)$ is given by

$$
(d \sigma)=\langle d \sigma\rangle_{0} e^{\int \phi_{k} d x^{k}} \text {, }
$$


where $(d \sigma)_{0}$ is some initial viaue of the element of arc.

Now suppose an equilibrium, or reversible, state is desired so that $\mathrm{dq}^{0} \equiv 0$.

Thus the desired condition is a null trajectory of the $\left(\mathrm{dq}^{0}\right)^{2}$ manifold. Then, if $f \neq 0$, the desired condition is also a null trajectory of the $(d \sigma)^{2}$ manifold.

This implies that

$$
d(d \sigma)=0
$$

or

$$
(d \sigma)=(d \sigma)_{0}
$$

so that

$$
e^{\int \phi_{k} d x^{k}}=1
$$

which is satisfied only if

$$
\int \phi_{k} d x^{k}=2 \pi i n,
$$

where $n$ is an integer. This is the quantum condition London introduced.

To illustrate how this condition arises from the dynamic approach, suppose a description of a hydrogen atom is desired. A hydrogen atom is in a stable condition and, if isolated, satisfies che conditions $\overline{\mathrm{d}} E=0$ and $\mathrm{dq} q^{0}=0$. These conditions arong with $f \neq 0$ establish the quantization of the integral $\int \phi_{k} d x^{k}$.

To show how the Dynamic Theory removes from London's work the difficulty of defining length as a real number, consider an elementary presentation of London's. Suppose the field of a proton to be given by

$$
\phi_{0}=\frac{\alpha^{1}}{r} ; \phi_{i} \equiv 0 ; i \neq 0 .
$$

Equality of forces for the simple case of circular motion requires that 


$$
\frac{m v^{2}}{r}=\frac{e^{2}}{r^{2}}
$$

Thus the period is given by $T=\frac{2 \pi r}{v}$ and the velocity by

$$
v=\frac{e}{\sqrt{m r}}
$$

Now:

$$
\int \phi_{k} d x^{k}=\int \phi_{0} c d t=\phi_{0} c T=2 \pi i n,
$$

so that

$$
\frac{\alpha^{1} c T}{r}=\alpha^{1} c 2 \pi \sqrt{\frac{m r}{e}}=2 \pi i n .
$$

Solving for the radius shows that the allowed radii are

$$
r=\frac{-n^{2} e^{2}}{\alpha^{12} m c^{2}}
$$

By choosing

$$
\alpha^{1}=\frac{2 \pi i e^{2}}{h c}=\frac{i}{137} \equiv i \alpha,
$$

where $h$ is Planck's constant, then the possible radij become

$$
r=\frac{n^{2} h^{2}}{4 \pi^{2} e^{2} m}
$$

which are the Bohr radii. 
The imaginary $\alpha^{1}$ presented the difficulty, in London's work, of defining length as a real number. In the dynamic approach real distance, or length, may be defined, and properly should be, in the ( $\left.\mathrm{dq}^{0}\right)$ manifold. Recalling that the definition of the potentials is

$$
\phi_{k}= \pm \frac{\partial \ln f^{\frac{1}{2}}}{\partial x^{k}}
$$

it may easily be seen that if $f<0$, then $\phi_{k}$ becomes imaginary as does the length of arc in the $\left(d_{\sigma}\right)^{2}$ manifold since the length of arc is given by

$$
\sigma=\int \sqrt{(\mathrm{d} \sigma)^{2}}
$$

However, the arc length in the $\left(d q^{0}\right)^{2}$ manifold is real since $d q^{0} \geq 0$ by the second law.

It should be noted that the conditions for quantization are not restricted to $\overline{\mathrm{d}} E=0, \mathrm{dq}^{0}=0$, and $\mathrm{f}<0$ as used here. Any set of conditions which results in the final element of arc $(d \sigma)$ being equal to the initial element of arc $\left(d_{\sigma}\right)_{0}$ results in quantum conditions. It is particularly significant to note that the quantization involves only forces which may be described in terms of the "distance curvatuve" and does not involve forces describable by a vector curvature. Thus interpreting the gauge potentials $\phi_{k}$ to be electromagnetic potentials provides quantum effects for electromagnetic forces. Interpreting the forces describable by the vector curvature to be gravitational removes the possibility of achieving quantum effects involving gravitational forces alone.

Here, again, is a distinction between curved and Euclidean manifolds, though here it appears slightly different. The Dynamic Theory requires a quantization. However, this quantization cepends upon the existence of a gauge function and appropriate restrictive conditions. Thus a curved space may exhibit quantum effects but only if the curvature is accompanied by a gauge function or a distance curvature.

Thus the Dynamic Theory, through London's quantization, not only supports the contention that "God does not play with dice all the time" but, further, may supply the answers to two questions concerning quantum physics. The first 
question is, "What is waving in the wave function?" London showed that the wave function is directly related to the element of the arc length in the sigma manifold. Therefore the "waving" is the tendency of this element of arc length to increase and decrease around a closed path. Using the calculus of complex variables, the quantum number becomes the order, or multiplicity of the zero of (do).

The second question is how gravitational effects mav be quantized? Here the answer becomes, "It can not." If we assume the validity of Einstein's General Theory of Relativity, which is included within the scope of the Dynamic Theory, in equating gravitational effects with the curvature of the space-time manifold, then gravitational effects alone may not be quantized. However, electromagnetic effects within a gravitational field may still be quantized. However, the quantization will be affected by the vector curvature.

\section{G. Summary}

When this investigation was initiated, it was concluded that Einstein's postulate of the constancy of the velocity of light could not be adopted since it was felt that experimental evidence in electromagnetism alone did not justify applying it as a limiting velocity to all types of forces. However, we find that this is required by the Dynamic Theory which approaches physical phenomena from a different way. The new viewpoint indeed supports Einstein's every contention including his uneasiness concerning quantization and it does it in such a way that it seems only the early successes of his theories kept Einstein himself from coming to the same realization.

This is, of course, speculation, but it was Einstein who returned to very fundamental concepts in order to establish a basis for his relativity theory. He was also known to be aware of the tremendous strength of classical thermodynamics since he wrote, "A theory is the more impressive the greater the simplicity of its premises are, the more different kinds of things it relates, and the more extended is its area of applicability. Therefore the deep impression that classical thermodynamics made upon me. It is the only physical theory of universal content concerning which I am convinced that, within the framework of applicability of its basic concepts, it will never be overthrown." Thus it seems only the fact that Caratheodory's statement of the second law, which is the key to the development of the Dynamic Theory, did not make its appearance before the relativistic theory had achieved such stupendous successes kept Einstein from eventually investigating its possible extended application. 
The key points in the development of the Dynamic Theory seem to be the recognition of the generality of the thermodynamic laws and their independence upon the number or type of variables considered and the recognition that the quadratic forms associated with the stability conditions form natural metrics leading to a geometrical description of the dynamics of the system independent of the variables used in the description.

There are numerous conclusions and implications that could be reiterated here; however, only a few of the seemingly more significant ones will be discussed. The first one is the existence of an integrating factor for any system describable by the first law, particularly an integrating factor independent of the type of force considered. It is this fact which ultimately leads to a unique 1 imiting velocity for all forces. However, in speaking of the absolute velocity for mechanical systems, care must be taken to point out that, as far as the three laws are concerned, it does not represent an absolute barrier. Rather the laws only state that, for a mechanical system with only three work terms representing the work done by three spatial forces the absolute velocity represents an upper and lower limit. Thus solutions with velocities greater than the speed of light are also allowed. However, so long as the system is subjected to only these three forces, then its velocity may never cross this barrier. This absolute barrier effect may be expected to change if another force term representing an additional dimension is found necessary.

The reduction in the number of fundamental laws or postulates is significant. This together with the unifying effect of the three laws promises to simplify the study of physical phenomena by founding the entire realm of physics upon a common set of conceptualizations.

Perhaps the best way to summarize the unifying effect of the Dynamic Theory is to consider a Venn diagram that depicts not only the overall realm of applicability of the theorem, but indicates also the effects of different restrictions. 


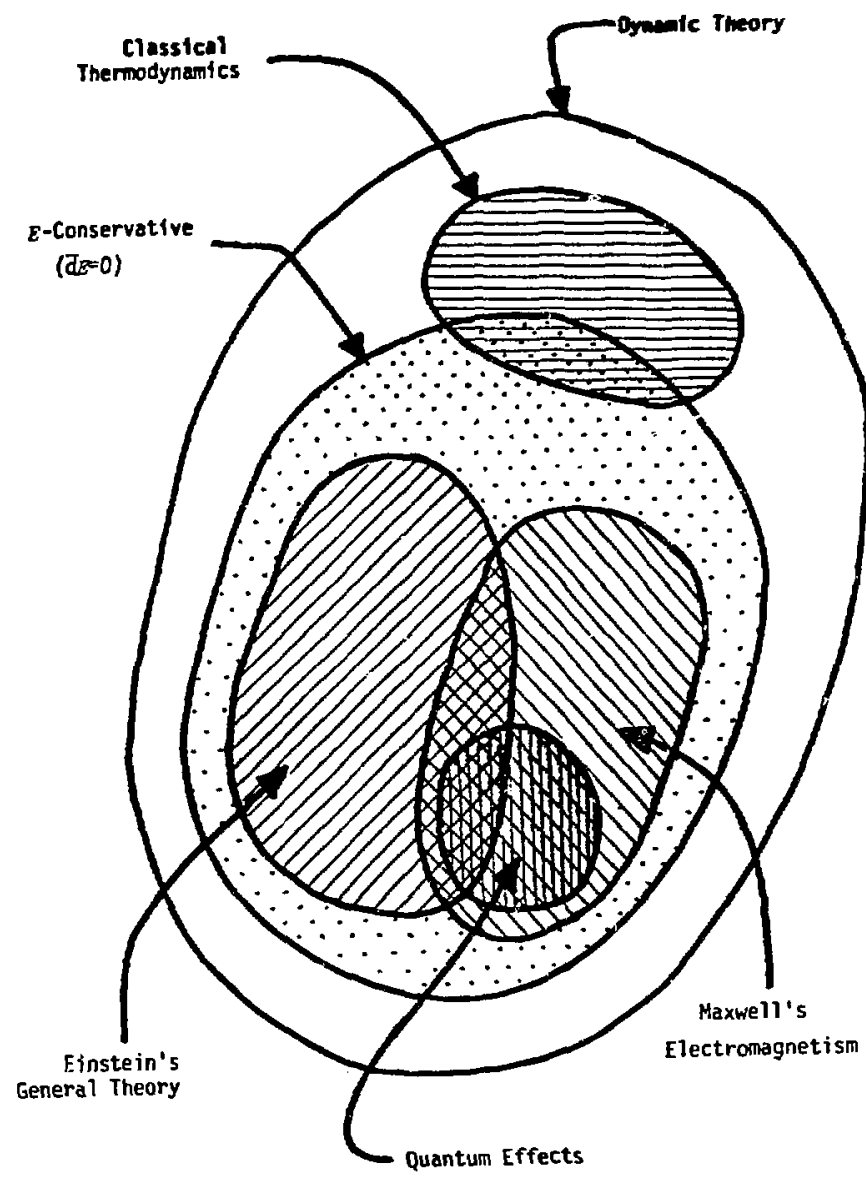

\section{FIVE-DIMENS IONAL SYSTEMS}

During the preceding development displaying the unifying effect of the Dynamic Theory, there did not appear to be anything that approached a description of nuclear effects. Of course quantum theorists may respond that the nuclear effects lie within the realm of quantum the ory. This, however, does not seem to be a strong argument since current nuclear theory appears to depend upon a number of ad hoc postulates.

If it is supposed that nuclear theory cannot be extracted from some aspect of the preceding four-dimensional world view, then how might the Dynamic Theory produce a foundation for nuclear theory? At this point there may appear to be no obvious way. Therefore let us proceed on a different tack. 
Thus far we have constantly adhered to the policy of dividing systems into two types: thermodynamic systems with only a work term of the $p$ dV type and mechanical systems with three mechanical, or spatial, work terms. Now the generality of the adopted laws places no restrictions upon the number or type of variables used. Particularly there is no restriction coming from the laws themselves which says we cannot use four work terms, one the thermodynamic $p d V$ termi and three mechanical $F$ dq terms. Obviously $p$ dV itself is just another $F d q$ type term with the pressure as the generalized force and the volume as the generalized displacement.

The rub comes in attempting to visualize a world description in five dimensions. Many arguments may be envisioned which tend to imply only a fourdimensional manifold is needed. The kinetic theory of gases relates the pressure to the average velocities of the particies contained. Does that not imply that thermodynamics ultimately rests on a four-dimensional manifold? Recall that the system in the kinetic theory is basically in equilibrium.

Statistical thermodynamists may claim that thermodynamics is basically statistical in nature and is fundamentally tied to order and disorder and hence to the four-dimensional world of quantum theory. But remember that the overall system, to which the statistical approach is applicable, is a composite system made up of many subsystems each in an equilibrium state.

Still there seems to be no substantial support for a five-dimensional world from the point of view of current theories. This is to be expected though in view of the difficulties experienced in the transition from the classical threedimensiorial world to the four-dimensional space-time of Einstein's theories. Obviously had the extension of the universe been restricted on a priori grounds to three--dimensional Euclidean space, Einstein's theory would have been rejected on first principles. On the other hand as soon as we recognize that the fundamental continuum of the universe and its geometry cannot be posted a priori and can only be disclosed to us from place to place by experiment and measurement, a vast number of possibilities are thrown open. Among these the four-dimensional space-time of relativity, with its varying degrees of non-Euclideanism, has found a place. So also may the five-dimensional view of the Dynamic Theory be found within the possibilities. Ultimate judgment upon its necessity, or applicability, should rest upon a comparison of the theory's predictions with reality. 


\section{A. Systems Near an Equilibrium State}

The metric coefficients are made up of the second partial derivatives of the system energy function and therefore if the system remains near an equilibrium state, then the value of these derivatives evaluated at the equilibrium state may be used as a first approximation for the metric coefficients. In this case the geometry will be Euclidean and, from the preceding four-dimensional development, the Euclidean manifold produced by applying the E-conservative restriction was Minkowski's space-time continuum of special relativity.

Therefore suppose we begin an investigation of the five-dimensional world by staying very near an equilibrium state so as to simplify the description to a five-dimensional gereralization of Minkowski's space-time manifold.

1. Equations of Motion. Suppose that we consider some sort of system requiring four work terins and for the moment not concern ourselves as to exactly what this system might be. Thus for our system we will have thermodynamic as well as mechanical variables and the first law becomes

$$
\overline{\mathrm{d}} \tilde{E}=\mathrm{d} \tilde{U}+P d v-\tilde{F}_{\alpha} d q^{\alpha} ; \alpha=1,2,3 .
$$

Where the $\tilde{E}, \tilde{U}, \vee$ and $\tilde{F}_{\alpha}$ are considered as specific quantities. That is, these quantities are related to a unit of mass such as is customary in thermodynamics.

The specific volume is the reciprocal of the mass density i; using the mass density instead of the specific volume the first law becomes

$$
\widetilde{d E}=\mathrm{d} \tilde{U}-\left(\mathrm{P} / \gamma^{2}\right) \mathrm{d} \gamma-F_{\alpha} d q^{\alpha} ; \alpha=1,2,3 .
$$

This law now requires that the system's specific energy $\tilde{U}$ be a function of five independent variables so that

$$
\tilde{U}=\tilde{U}\left(\tilde{S}, q^{1}, q^{2}, q^{3}, \gamma\right)
$$

Thus the first law requires a five-dimensional manifold of specific eritropy, space, and mass density for a general system. Since the system under cons ideration needs both thermodynamic and mechanical variables, we can no longer refer 
to the entropy as mechanical or thermodynamic; however, the limiting case where the mass is held fixed must produce the mechanical entropy.

The procedure established by the Dynamic Theory is to take the stability condition quadratic form as the metric for a stable system. Thus the coefficients of the metric become the second partial derivatives of the energy function. In order to simplify the metric, suppose for the present that we restrict our system to be very near an equilibrium state so that we may consider the second partial derivatives to be constants. This is in essence considering a local Euclidean manifold; the symmetry of the geometric connections guarantees that we may do this.

Since the metric coefficients are constants, a transformation may be found such that the cross terms are zero. Then in this coordinate system and when

$$
q^{0} \equiv \frac{S}{F_{0}} \quad \text { and } \quad q^{4} \equiv \frac{\gamma}{a_{0}} \text {, }
$$

the metric becomes

$$
c^{2}(d t)^{2}=\left(d q^{0}\right)^{2}+d q^{\alpha} d q^{\alpha}+\left(d q^{4}\right)^{2} \text { with } \alpha=1,2,3 .
$$

If we again consider the restriction $\overline{\mathrm{d}} \tilde{E}=0$ so that we are talking of an $E$-conservative system for which the principle of increasing entropy hoids, then we have the variational principle given by

$\delta \int \sqrt{(\mathrm{d} S)^{2}}=0$

Solving Eq. (58) for $\mathrm{dq}^{0}$ and squaring we get

$$
\left(d q^{0}\right)^{2}=c^{2}(d t)^{2}-d q^{\alpha} d q^{\alpha}-\left(d q^{4}\right)^{2}
$$

or

$$
\left(\frac{d q^{0}}{d t}\right)^{2}=c^{2}-g \alpha \beta\left(\frac{d q^{\alpha}}{d t}\right)\left(\frac{d q^{\beta}}{d t}\right) ; \alpha, \beta=1,2,3,4
$$




$$
g \alpha \beta=\delta \alpha \beta .
$$

The entropy manifold given by Eq. (60) is a five-dimensional Minkowski-type manifold with coordinates of space-time-mass. We may therefore follow the procedure Minkowski and Einstein used in the Special Theory of Relativity.

First, to avoid confusion, let us rename the coordinates as

$$
x^{0} \equiv \text { ct } ; \quad x^{1} \equiv q^{1}, x^{2} \equiv q^{2}, x^{3} \equiv q^{3}, \quad \text { and } x^{4} \equiv q^{4} \text {. }
$$

Then define the five-dimensional velocity vector as

$$
u^{i} \equiv \frac{d x^{i}}{d q^{0}} ; i=0,1,2,3,4
$$

and define the five-dimensional acceleration vector as

$$
f^{i} \equiv \frac{\delta u^{i}}{\delta q^{0}} \equiv \frac{d^{2} x^{i}}{d q^{0}}+\left\{\begin{array}{l}
0_{k}^{i} \\
\}
\end{array} \frac{d x^{\ell}}{d q^{0}} \frac{d x^{k}}{d q^{0}} .\right.
$$

Now the specific entropy is the arc length and the variational principle is based upon the entropy. Therefore if we multiply the specific entropy by the mass density, we have the entropy density. The variational problem becomes

$\delta \int \sqrt{\gamma^{2}\left(\mathrm{dq}^{0}\right)^{2}}=\delta \int \gamma \sqrt{\left(\mathrm{dq}^{0}\right)^{2}}=0$.

The Euler equations for this problem are

$$
\frac{d}{d q^{0}}\left\{\frac{\gamma g_{k j} u^{j}}{\sqrt{g_{i j} u^{i} u^{j}}}\right\}-\frac{\partial \gamma}{\partial x^{k}} \sqrt{g_{i j} u^{i} u^{j}}-\frac{\gamma \frac{\partial g_{i k}}{\partial x^{k}} u^{i} u^{j}}{\sqrt{g_{i j} u^{i} u^{j}}}=0
$$

or 


$$
a^{0} u^{4}\left[\frac{g_{i j} u^{j}}{\sqrt{g_{i j} u^{i} u^{j}}}\right]-\frac{\partial \gamma}{\partial x^{i}} \sqrt{g_{i j} u^{i} u^{j}}+\gamma\left\{\frac{d}{d q^{0}}\left[\frac{g_{i j} u^{j}}{\sqrt{g_{i j} u^{i} u^{j}}}\right]-\frac{\frac{\partial g_{i k}}{\partial x^{i}} u^{i} u^{k}}{\sqrt{g_{i j} u^{i} u^{j}}}\right\}=0 .
$$

Using the fact that $g_{i j} u^{j} u^{j}=1$, the Euter equations become

$$
\gamma f^{i}=\frac{\partial \gamma}{\partial x^{i}}-a_{0} u^{4} g_{i j} u^{j} \equiv F^{i}
$$

where the $\mathrm{F}^{i}$ are force densities.

Obviously if we hold the mass density fixed, $u^{4} \equiv 0$, then the volume integral of this equation becomes the force-mass-acceleration relationship of special relativity.

Now since $f^{i}=\frac{\delta u^{i}}{\delta q^{0}}$ and $\left(\frac{d q^{0}}{d t}\right)=c^{2}-u^{\alpha} u^{\alpha} ; \alpha=1,2,3,4$,

then

$$
\begin{aligned}
F^{i} & =\gamma \frac{\delta u^{i}}{\delta q^{0}}=\gamma \frac{\delta u^{i}}{\delta t} \frac{d t}{d q^{0}} \\
& =\frac{\gamma}{\sqrt{c^{2}-v^{2}}} \frac{\delta}{\delta t}\left(\frac{d x^{i}}{d q^{0}}\right) \text { where } v^{2}=u^{\alpha} u^{\alpha} ; \alpha=1,2,3,4 .
\end{aligned}
$$

Then

$$
\begin{aligned}
F^{\alpha} & =\frac{\gamma}{\sqrt{c^{2}-v^{2}}} \frac{s}{\delta t}\left(\frac{1}{\sqrt{c^{2}-v^{2}}} \frac{d x^{\alpha}}{d t}\right) \\
& =c^{2} \frac{\gamma}{\sqrt{1-\beta^{2}}} \frac{\delta}{\delta t}\left(\frac{1}{\sqrt{1-\beta^{2}}} \frac{d x^{\alpha}}{d t}\right),
\end{aligned}
$$


where $\beta \equiv v / c$ with $v$ the four-dimensiona $;$ speed.

The force density equation may now be written as

$$
\sqrt{1-\beta^{2}} F^{\alpha}=\frac{\gamma}{c^{2}} \frac{\delta}{\delta t}\left(\frac{1}{\sqrt{1-\beta^{2}}} \frac{d x^{\alpha}}{d t}\right) .
$$

Consider

$$
\frac{\delta}{\delta t}\left[\frac{\gamma}{\sqrt{1-\beta^{2}}} \frac{d x^{\alpha}}{d t}\right]=\frac{\delta}{\delta t}(\gamma)\left[\frac{1}{\sqrt{1-\beta^{2}}} \frac{d x^{\alpha}}{d t}\right]+\frac{\gamma \delta}{\delta t}\left[\frac{1}{\sqrt{1-\beta^{2}}} \frac{d x^{\alpha}}{d t}\right] ;
$$

but $\frac{\delta(\gamma)}{\delta t}=a_{0} v^{4}$, so that the force density equations may now be written as

$$
\sqrt{1-\beta^{2}} F^{\alpha}=\frac{1}{c^{2}} \frac{\delta}{\delta t}\left[\frac{\gamma}{\sqrt{1-\beta^{2}}} \frac{d x^{\alpha}}{d t}\right]-\frac{a_{0} u^{4}}{c^{2}}\left[\frac{1}{\sqrt{1-\beta^{2}}} \frac{d x^{\alpha}}{d t}\right] .
$$

We may define $\gamma /\left(\sqrt{1-\beta^{2}}\right) \equiv \gamma^{1}$ as the effective mass density or "relativistic" mass density; then

$$
\sqrt{1-\beta^{2}} F^{\alpha}=\frac{1}{c^{2}} \frac{\delta}{\delta t}\left[\gamma^{1} \frac{d x^{\alpha}}{d t}\right]-\frac{{ }^{a} v^{4} v^{\alpha}}{c^{2} \sqrt{1-\beta^{2}}}
$$

by defining $F^{\alpha} \equiv c^{2} \sqrt{1-\beta^{2}}\left(F^{\alpha}\right)$ so that

$$
F^{\alpha}=\frac{\delta}{\delta t}\left[\gamma^{1} \frac{d x^{\alpha}}{d t}\right]-\frac{a_{0} v^{4} v^{\alpha}}{\sqrt{1-\beta^{2}}}
$$

We see that this force density becomes Einstein's special relativistic force density when $v^{4}=0$, or for constant "rest mass." Thus the equations of motion, Eq. (63), reduce to Einstein's special relativistic equations of motion when $\dot{\gamma} \equiv 0$. 
2. Energy Equation. Now for our system the restriction that

$$
\widehat{\mathrm{d}} \tilde{E}=0=\mathrm{d} \tilde{U}-\frac{\mathrm{p}}{\gamma^{2}} \mathrm{a} \gamma-\tilde{F}_{\alpha} \mathrm{dx}{ }^{\alpha}, \alpha=1,2,3,
$$

requires that

$$
\mathrm{d} \tilde{U}=\frac{\mathrm{p}}{\gamma^{2}} \mathrm{~d} \gamma+F_{\alpha} \mathrm{dx} x^{\alpha}, \alpha=1,2,3,
$$

or if $\frac{p}{\gamma^{2}}$ is considered as another generalized force density, then

$$
\mathrm{d} \tilde{U}=\tilde{F}_{\alpha} \mathrm{dx}{ }^{\alpha}, \alpha=1,2,3,4 .
$$

Thus by integrating the expression for the system's specific energy change, we should arrive at the Einstein energy equation if we hold $\dot{u}^{4} \equiv 0$. Therefore we shall perform the integration using the force densities given by Eq. (63) to get the system's energy, or

$$
\begin{aligned}
\tilde{U}-\tilde{U}_{0} & =\int_{p_{0}}^{p} \tilde{F}_{\alpha} d x^{\alpha}=\int_{p_{0}}^{p}\left\{\frac{d}{d t}\left[\frac{\gamma}{\sqrt{1-\beta^{2}}} \frac{d x^{\alpha}}{d t}\right]-\frac{a_{0} u^{4} u^{\alpha}}{\sqrt{1-\beta^{2}}}\right\} d x^{\alpha} \\
& =\int_{t_{0}}^{t}\left\{\gamma\left[\frac{d}{d t}\left(\frac{1}{\sqrt{1-\beta^{2}}}\right) u^{\alpha} u^{\alpha}+\frac{u^{\alpha} \frac{d u^{\alpha}}{d t}}{\sqrt{1-\beta^{2}}}\right]\right\} d t .
\end{aligned}
$$

But $c^{2} \beta^{2}=u^{\alpha} u^{\alpha}$ and $c^{2} \dot{\beta} \dot{\beta}=u^{\alpha} \frac{d u^{\alpha}}{d t}$; therefore,

$$
\begin{aligned}
\tilde{U}-\tilde{U}_{0} & =\int_{t_{0}}^{t}\left\{\gamma\left[\frac{d}{d t}\left(\frac{1}{\sqrt{1-\beta^{2}}}\right) c^{2} \beta^{2}+\frac{c^{2} \beta \dot{\beta}}{\sqrt{1-\beta^{2}}}\right]\right\} d t \\
& =c^{2} \int_{\beta_{0}}^{\beta} \gamma \frac{\beta d \beta}{\left(1-\beta^{2}\right)^{3 / 2}} .
\end{aligned}
$$


Now $\beta$ depends upon $u^{\alpha}$ and not upon $x^{4}$ or $\gamma$; therefore

$$
\tilde{U}-\tilde{U}_{0}=\frac{\gamma c^{2}}{\left(1-\beta^{2}\right)^{1 / 2}}
$$

or

$$
\tilde{U}=\frac{\gamma c^{2}}{\left(1-\beta^{2}\right)^{1 / 2}}+\text { constant } .
$$

If the internal energy is considered as the system's energy when the spatial velocities $u^{\alpha} ; \alpha=1,2,3$ are taken as zero, then the internal energy density given by

$$
\tilde{U}=\frac{\gamma c^{2}}{\sqrt{1-\left(\frac{u^{4}}{c}\right)^{2}}}+\text { constant } .
$$

At the equilibrium condition where $u^{4}$ is also zero the internal energy density is then

$$
\tilde{U}=\gamma c^{2}+\text { constant }
$$

By taking the constant of integration to be zero, this internal energy density then corresponds to Einstein's "rest energy" where here the "rest energy" is in terms of a four-dimensional "at rest" state.

If we make the usual approximation of allowing $\beta^{2} \ll 1$, then the system's energy density is approximately given by

$$
\tilde{U}=\gamma c^{2}+\frac{1}{2} \gamma v^{2}+\frac{1}{2} \frac{\gamma}{\left(a_{0}\right)^{2}}(\dot{\gamma})^{2},
$$

where here $u^{4} \equiv \frac{\dot{y}}{a_{0}}$ is used. This displays the classical limit system energy density for an $E$-Conservative system very near equilibrium. 


\section{B. Systems With Non-Euclidean Manifold}

Suppose now we relax the assumption that the system is very near an equilibrium point so that the second partial derivatives are no longer constants but are functions. This is essentially the same transition as Einstein made going from his special to general theory; however, the logic of the transition is much simpler here. The only change in the logic appears in the relaxation of the assumption of nearness. There is, of course, a drastic increase in mathematical difficulty since the metric components are no longer constants.

1. General Variational Principle. We shall consider a system, which may be a charged plasma, that must be described by both thermodynamic and mechanical variables. When written in terms of the mass density, the first law for this system may be written as

$$
\mathrm{d} \tilde{E}=\mathrm{d} \tilde{U}-\frac{\mathrm{P}}{\gamma^{2}} \mathrm{~d} \gamma-\tilde{F}_{\sigma} \mathrm{dq}^{\alpha}, \alpha=1,2,3,
$$

where the tilde denotes specific quantities.

Following the prescribed procedures of the Dynamic Theory we shail take the stability condition quadratic form as the metric for our system. Thus the metric coefficients will be given by the second partial derivatives

$$
h_{i j}=\frac{\partial U^{2}}{\partial q^{i} \partial q^{j}}, j, j=0,1,2,3,4 \text {, }
$$

where $q^{4} \equiv \frac{\gamma}{a_{0}}$. The metric may then be written as

$$
c^{2}(d t)^{2}=h_{00}\left(d q^{0}\right)^{2}+2 h_{0 \alpha} d q^{0} d q^{\alpha}+h_{\alpha \beta} d q^{\alpha} d q^{\beta},
$$

where $\alpha, \beta=1,2,3,4$.

Imposing the restriction that the system be $E$-conservative, $\overline{\mathrm{d}} E=0$, results in the principle of increasing entropy, so that

$$
\delta \int \sqrt{(d s)^{2}}=0
$$


Thus in terms of the specific entropy the variational principle may be written as

$$
\delta \int \sqrt{\left(\gamma d q^{0}\right)^{2}}=\delta \int \gamma \sqrt{\left(d q^{0}\right)^{2}}=0
$$

Solving the metric given by Eq. (67) and squaring yields the expression

$$
\left(d q^{0}\right)^{2}=\left\{\frac{1}{h_{00}}\right\}\left\{c^{2}(d t)^{2}+2 h_{0 \alpha}[*] d t d q^{\alpha}-h_{\alpha \beta} d q^{\alpha} d b^{\beta}\right\}, \alpha, \beta=1,2,3,4,
$$

with

$$
[*] \equiv \frac{h_{0 \gamma}}{h_{00}} \dot{q}^{\gamma} \pm \sqrt{\frac{c^{2}}{h_{00}}-\frac{h_{\gamma \delta}}{h_{00}} \dot{q}^{\gamma} q^{\delta}+\frac{\left(h_{0 \gamma} \dot{q}^{\gamma}\right)^{2}}{\left(h_{00}\right)^{2}}} \text {. }
$$

This metric in a five-dimensional manifold of space-time-mass may be rewritten as

$$
\left.(\mathrm{dq})^{0}\right)^{2}=\left(\frac{1}{\mathrm{~h}_{00}}\right)(\mathrm{d} \sigma)^{2},
$$

where

$$
\left(d q^{0}\right)^{2} \equiv \hat{q}_{i j} d x^{i} d x^{j}, i, j=0,1,2,3,4,
$$

and

$$
(d \sigma)^{2} \equiv \hat{\hat{q}}_{i j} d x^{i} d x^{j}, \quad i, j=0,1,2,3,4,
$$

with $x^{0} \equiv c i, x^{1} \equiv q^{1}, x^{2} \equiv q^{2}, x^{3} \equiv q^{3}$, and $x^{4} \equiv \gamma / a_{0}$. Thus we may write

$$
\left(d q^{0}\right)^{2}=\hat{q}_{i j} d x^{i} d x^{j}=\left(\frac{1}{f}\right)(d \sigma)^{2}=\left(\frac{1}{f}\right) \hat{\hat{g}}_{i j} d x^{i} d x^{j}
$$

86 
Having established the metrics in Eq. (64) in the manner prescribed by the Dynamic Theory, the geometry must be Weyl geometry; defining the potential fivevector as

$$
\phi_{i} \equiv \pm \frac{\partial \operatorname{lnf} f^{1 / 2}}{\partial x^{i}}
$$

and the field tensor as

$$
F_{i j} \equiv \phi_{i, j}-\phi_{j, i}
$$

then we may follow Weyl's procedure in his unified field theory to arrive at the variational principle

$$
\delta \int\left[\hat{\mathrm{R}}+\frac{1}{2} A \tilde{\mathrm{F}}_{i j} \tilde{\mathrm{F}}^{i j}-\lambda\left(\frac{1}{2}-12 \tilde{\phi}_{j} \tilde{\phi}^{i}\right)\right] \gamma-\overline{\hat{\mathrm{g}}} \mathrm{dx} x^{5}=0
$$

where $\tilde{F}_{i j} \equiv \frac{1}{\sqrt{\lambda}} F_{i j}$ and $\tilde{\phi}_{i} \equiv \frac{1}{\sqrt{\lambda}} \phi_{i}$.

Varying the metric coefficients $\hat{\hat{g}}_{i j}$ in the variational principle (67) will yield field equations of the Dynamic. Theory which are extensions of Einstein's General Theory of Relativity.

2. Gauge Function Field Equations. In order to isolate the field equations resulting from a gauge function from the field equations produced by a vector curvature, let us consider a local Euclidean manifold for $(d \sigma)^{2}$.

Now the field tensor given by Eq. (66) has 25 components. We would like to deter.: ne the field equations for these components. The quickest, though not the only, way is to consider the five dimensions to be

$$
x^{0}=i c t ; x^{\alpha}=x^{\alpha}, \alpha=1,2,3,4 .
$$

The field tensor is then defined to be 


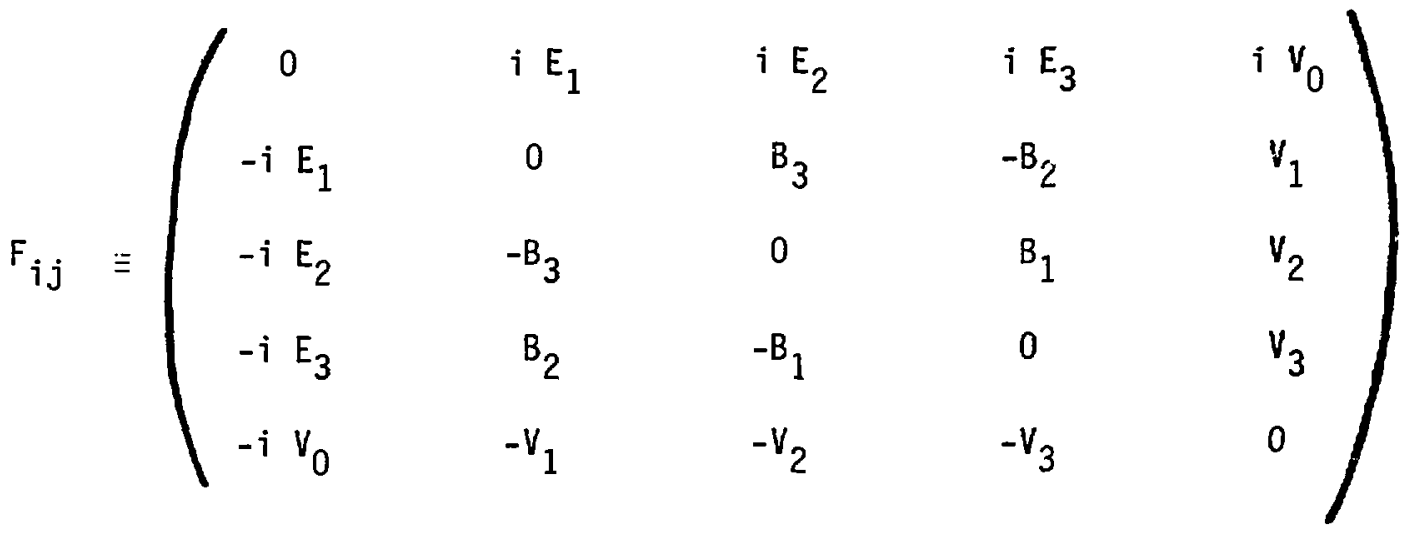

Using Bianchi's identities

$\frac{\partial F_{i j}}{\partial x^{k}}+\frac{\partial F_{j k}}{\partial x^{i}}+\frac{\partial F_{k j}}{\partial x^{j}}=0$

and the various combinations of the indices $0,1,2,3,4$ we obtain the field equations

$$
\begin{aligned}
& \bar{\nabla} \cdot \bar{B}=0, \quad \bar{\nabla} \times \bar{E}+\frac{1}{c} \frac{\partial \bar{B}}{\partial t}=0, \\
& \bar{\nabla} \times \bar{V}+a_{0} \frac{\partial \bar{B}}{\partial \gamma}=0, \quad \text { and } \bar{\nabla} v_{0}+\frac{1}{c} \frac{\partial \bar{V}}{\partial t}+a_{0} \frac{\partial \bar{E}}{\partial \gamma}=0 .
\end{aligned}
$$

The definition of the five-vector current density

$$
\frac{\partial F_{i j}}{\partial x^{i}} \equiv \frac{4 \pi}{c} J_{i}
$$

yields the equations

$$
\begin{aligned}
& \bar{\nabla} \cdot \bar{E}+a_{0} \frac{\partial V_{0}}{\partial \gamma}=4 \pi \rho, \bar{\nabla} \times \bar{B}-\frac{1}{c} \frac{\partial \bar{E}}{\partial t}+a_{0} \frac{\partial \bar{V}}{\partial \gamma}=\frac{4 \pi \bar{J}}{c}, \\
& \text { and } \bar{\nabla} \cdot \bar{V}+\frac{1}{c} \frac{\partial V_{0}}{\partial t}=-\frac{4 \pi}{c} J_{4} .
\end{aligned}
$$


Equations (68) and (70) form a set of seven Maxwel1-type equations which obviously reduce to Maxwell's four equations if the mass density is held fixed. The wave equations for the new field quantities may be derived using stanard assumptions.

$$
\frac{\partial}{\partial t}(\bar{\nabla} \cdot \bar{\nabla})+\frac{1}{c} \frac{\partial^{2} V_{0}}{\partial t^{2}}=\frac{4 \pi}{c} \frac{\partial J_{4}}{d t}=\bar{\nabla} \cdot \frac{\partial \bar{V}}{d t}+\frac{1}{c} \frac{\partial^{2} V_{0}}{\partial t^{2}}
$$

and

$$
\bar{\nabla} \cdot\left(\bar{\nabla} v_{0}\right)+\frac{1}{c} \bar{\nabla} \cdot \frac{\partial \bar{V}}{\partial t}=-a_{0} \bar{\nabla} \cdot \frac{\partial \bar{E}}{\partial y}=\frac{1}{c} \bar{\nabla} \cdot \frac{\partial \bar{V}}{d t}+\bar{\nabla} \cdot \bar{\nabla} v_{0} \cdot
$$

Therefore,

$$
\nabla^{2} V_{0}-\frac{1}{c^{2}} \frac{\partial^{2} V_{0}}{\partial t^{2}}=\frac{4 \pi}{c^{2}} \frac{\partial J_{4}}{\partial t}-a_{0} \bar{\nabla} \cdot \frac{\partial \bar{E}}{\partial \gamma}
$$

For the vector field we have:

$$
\bar{\nabla}(\bar{\nabla} \cdot \bar{V})+\frac{\bar{\nabla}}{c} \frac{\partial V_{0}}{\partial t}=-\bar{\nabla}\left(\frac{4 \pi}{c} J_{4}\right)
$$

and

$$
\bar{\nabla} \times(\bar{\nabla} \times \bar{v})+\nabla^{2} \bar{v}+\frac{1}{c} \frac{\partial}{\partial t} \bar{\nabla} v_{0}=\frac{4 \pi}{c} \bar{\nabla} J_{4} ;
$$

therefore

$$
\nabla^{2} \bar{v}-\frac{1}{c^{2}} \frac{\partial^{2} \bar{v}}{\partial t^{2}}=\frac{4 \pi}{c} \bar{\nabla} J_{4}+\frac{a_{0}}{c} \frac{\partial^{2} \bar{E}}{\partial t^{2 \gamma}}+a_{0}\left(\bar{\nabla} \times \frac{\partial \bar{B}}{\partial \gamma}\right)
$$

89 
But $\bar{\nabla} \cdot \bar{E}=4 \pi \rho-a_{0} \frac{\partial V_{0}}{\partial \gamma}$, so that

$$
\nabla^{2} v_{0}-\frac{1}{c^{2}} \frac{\partial^{2} v_{0}}{\partial t^{2}}=\frac{4 \pi}{c^{2}} \frac{\partial J_{4}}{\partial t}-a_{0} \frac{\partial}{\partial \gamma} 4 \pi \rho-a_{0} \frac{\partial v_{0}}{\partial t}
$$

and $\bar{\nabla} \times \bar{B}-\frac{1}{c} \frac{\partial \bar{E}}{\partial t}=\frac{4 \pi}{c} \bar{J}-a_{0} \frac{\partial \bar{V}}{\partial \gamma}$, so that

$$
\nabla^{2} \bar{V}-\frac{1}{c^{2}} \frac{\partial^{2} \bar{V}}{\partial t^{2}}=\frac{4 \pi}{c} \bar{\nabla} J_{4}+\hat{a}_{0} \frac{\partial}{\partial \gamma} \frac{4 \pi}{c} \bar{J}+2 \frac{\partial \bar{E}}{\partial t}-a_{0} \frac{\partial \bar{V}}{\partial \gamma}
$$

Now the wave equations for the usual vector and scalar potentials are

$$
\nabla^{2} \bar{A}-\frac{1}{c^{2}} \frac{\partial^{c} \bar{A}}{\partial t^{2}}=-\frac{4 \pi}{c} \bar{J}
$$

and

$$
\nabla^{2} \phi-\frac{1}{c^{2}} \frac{\partial^{2} \phi}{\partial t^{2}}=-4 \pi \rho
$$

We may differentiate these with respect to the mass density and substitute them into our wave equations and get

$$
\nabla^{2} V_{0}-\frac{1}{c^{2}} \frac{\partial^{2}}{\partial t^{2}} V_{0}=\frac{4 \pi}{c^{2}} \frac{\partial J_{4}}{\partial t}+a_{0}^{2} \frac{\partial^{2} v_{0}}{\partial \gamma^{2}}
$$


and

$$
\nabla^{2} \bar{V}-\frac{1}{c^{2}} \frac{\partial^{2} \bar{V}}{\partial t^{2}}=\frac{4 \pi}{c} \bar{\nabla} J_{4}+a_{0} \frac{\partial}{\partial \gamma}\left[2 \frac{\partial \bar{E}}{\partial t}-a_{0} \frac{\partial \bar{V}}{\partial \gamma}\right]
$$

where $V_{0} \equiv v_{0}+a_{0} \frac{\partial \phi}{\partial \gamma}$ and $\bar{V}=\bar{V}-a_{0} \frac{\partial \bar{A}}{\partial \gamma}$.

3. Interpretation of the Current Densities. For our system the conservation of charge becomes

$$
\frac{\partial \mathrm{J}_{i}}{\partial \mathrm{x}^{i}}=0, \quad i=0,1,2,3,4,
$$

so that

$$
\frac{\partial \rho}{\partial t}+\bar{\nabla} \cdot \bar{J}+a_{0} \frac{\partial J_{4}}{\partial \gamma}=0
$$

Thus we see that defining the current densities by Eq. (69) leads us to consider the new component of current density $\mathrm{J}_{4}$ which alters the conservation of charge equation, Eq. (72).

Since Eq. (69) defining the current densities involves an interpretation linking these equations to reality there seems to be no a priori reason for this defining relationship. Defining the current density in this manner introduces also the necessity of interpreting the new term $J_{4}$, which in turn requires changing our concept of conservation of charge to that of Eq. (72). While the extension to five dimensions may well require changing our concept of conservation of charge, just as the step from three to four dimensions required a change in the conservation of mass, it should be possible to appeal to experimentation to determine this requirement.

Suppose we look at the defining relations

$$
\frac{\partial F_{i j}}{\partial x^{i}} \equiv 0
$$


Then Eq. (68) becomes

and

$$
\bar{\nabla} \cdot \bar{E}=-a_{0} \frac{\partial V_{0}}{\partial \gamma}, \bar{\nabla} \times \bar{B}-\frac{1}{c} \frac{\partial \bar{E}}{\partial t}=-a_{0} \frac{\partial \bar{V}}{\partial \gamma},
$$

$$
\bar{\nabla} \cdot \bar{V}+\frac{1}{c} \frac{\partial V_{0}}{\partial t}=0
$$

so that we may define the charge density as

$$
\rho \equiv-\frac{a_{0}}{4 \pi} \frac{\partial V_{0}}{\partial \gamma}
$$

and the current density as

$$
\bar{J} \equiv-\frac{a_{0} c}{4 \pi} \frac{\partial \bar{V}}{\partial \gamma}
$$

Substituting Eqs. (75) and (76) into the remaining Eq. (74), we obtain

$$
\bar{\nabla} \cdot \bar{J}+\frac{\partial \rho}{\partial t}=0
$$

which is the classical conservation of charge equation.

Thus if we use the defining Eq. (69), we are faced with interpreting the new term $\mathrm{J}_{4}$, which has its origin in the thermodynamic variables of our system. If, however, we choose the defining relations (73), (75), and (76), we may keep our concept of conservation of charge but this requires us, by Eqs. (75) and (76) to consider current densities to have their origin in the thermodynamics of our system.

\section{Additional Developments of the Five-Dimensional Field} a. Energy-Momentum Tensor

If we follow the approach of relativistic electrodynamics, we may define the tensor $\{T\}$ in terms of the field tensor $\{F\}$ according to 


$$
T_{j k} \equiv\left(\frac{1}{4 \pi}\right)\left[F_{j \ell} F_{l k}+\frac{1}{4} \delta_{j k} F_{s t} F_{s t}\right]
$$

Recall that the five-dimensional field tensor is given by

$$
\{F\}=\left(\begin{array}{ccccc}
0 & i E_{1} & i E_{2} & i E_{3} & i V_{0} \\
-i E_{1} & 0 & B_{3} & -B_{2} & v_{1} \\
-i E_{2} & -B_{3} & 0 & B_{1} & V_{2} \\
-i E_{3} & B_{2} & -B_{1} & 0 & V_{3} \\
-i V_{0} & -V_{1} & -V_{2} & -V_{3} & 0
\end{array}\right)
$$

Using the field tensor to calculate the components of the energy-momentum tensor we find that the components are given by

$$
\begin{aligned}
& T_{0 \alpha}=\frac{-i}{4 \pi}\left[(\bar{E} \times \bar{B})_{\alpha}+V_{0} V_{\alpha}\right], \alpha=1,2,3, \\
& T_{00}=\frac{1}{8 \pi}\left[E^{2}+B^{2}+V_{0}^{2}+V^{2}\right], \\
& T_{04}=\frac{i}{4 \pi}[\bar{E} \cdot \bar{V}], \\
& T_{4 \alpha}=\frac{1}{4 \pi}\left[V_{0} E_{\alpha}+(\bar{V} \times \bar{B})_{\alpha}\right], \alpha=1,2,3, \\
& T_{44}=\frac{1}{8 \pi}\left[V_{0}^{2}+B^{2}-E^{2}-V^{2}\right],
\end{aligned}
$$

and

$$
T_{\alpha \beta}=\frac{1}{4 \pi}\left\{E_{\alpha \beta} E_{\beta}+B_{\alpha \beta} B_{\beta}-V_{\alpha} V_{\beta}-\frac{1}{2} \delta \alpha \beta\left[E^{2}+B^{2}+V_{0}^{2}-V^{2}\right]\right\},
$$

where $\alpha, \beta=1,2,3$.

93 
The field energy density may be defined by

$$
\xi \equiv \frac{1}{8 \pi}\left[\bar{E} \cdot \bar{E}+\bar{B} \cdot \bar{B}+\bar{V} \cdot \bar{V}+V_{0}^{2}\right]
$$

and the electrical Poynting vector may be defined by

$$
\bar{S}_{E} \equiv \frac{C}{4 \pi}(\bar{E} \times \bar{B})
$$

Now the electrical Poynting vector represents the outward flow of the electromagnetic field energy through a surface. Thus if we take the total vector, whose components are $T_{0 \alpha}$, to be the total flow of energy, then the vector with components $\frac{c}{4 \pi} V_{0} V_{\alpha}$ must be the outward flow of energy due to changes of the mass density within the surface. Therefore let us designate the mass energy vecter as

$$
\bar{S}_{\mathrm{m}} \equiv \frac{c}{4 \pi}\left(v_{0} \bar{v}\right)
$$

so that the total energy vector is

$$
\bar{S}=\bar{S}_{E}+\bar{S}_{m}
$$

whose components are

$$
S_{\alpha}=\frac{c}{4 \pi}\left[(\bar{E} \times \bar{B})_{\alpha}+V_{0} V_{\alpha}\right]=-\left(\frac{c}{\bar{i}}\right) T_{0 \alpha} .
$$

The Dynamic stress tensor may be defined as the three-dimensional tensor whose elements are

$$
T_{\alpha \beta}^{D}=\frac{1}{4 \pi}\left\{E_{\alpha \beta} E_{\beta}+B_{\alpha} B_{i}-V_{\alpha} V_{\beta}-\frac{1}{2} \delta_{\alpha \beta}\left[\xi-2 V^{2}\right]\right\} .
$$


The Maxwell stress tensor is defined in electrodynamics as the three-dimensional tensor with elements

$$
T_{\alpha \beta}^{M}=\frac{1}{4 \pi}\left\{E_{\alpha} E_{\beta}+B_{\alpha \beta} B_{\beta}-\frac{1}{2} \delta_{\alpha \beta}\left[E^{2}+B^{2}\right]\right\}
$$

Thus, in terms of the Maxwell stress tensor, the Dynamic stress tensor may be written as

$$
T_{\alpha \beta}^{D}=T_{\alpha \beta}^{M}-\frac{1}{4 \pi}\left\{v_{\alpha \beta} v_{\beta}-\frac{1}{2} \delta_{\alpha \beta}\left[v^{2}-v_{0}^{2}\right]\right\}
$$

Then in terms of the above defined quantities

$$
\{T\}=\left(\begin{array}{ccc}
\xi & -\frac{1}{c} \bar{S} & \frac{i}{4 \pi}(\bar{E} \cdot \bar{V}) \\
-\frac{i}{C} \bar{S} & \left\{T^{D}\right\} & \frac{i}{4 \pi}\left[V_{0} \bar{E}+(\bar{V} \times \bar{B})\right] \\
\frac{i}{4 \pi}(\bar{E} \cdot \bar{V}) & \frac{1}{4 \pi}\left[V_{0} \bar{E}+(\bar{V} \times \bar{B})\right] & \frac{1}{8 \pi}\left[V_{0}^{2}+B^{2}-E^{2}-V^{2}\right]
\end{array}\right)
$$

Suppose we calculate the trace of the energy-momentum tensor:

$$
\begin{aligned}
t_{r}\{T\}=T_{j j} & =T_{j j}^{D}+\xi+\frac{1}{8 \pi}\left[v_{0}^{2}+B^{2}-E^{2}-v^{2}\right] \\
& =\frac{1}{4 \pi}\left[B^{2}+v_{0}^{2}\right]+T_{\alpha \alpha}^{0} \\
& =\frac{1}{4 \pi}\left[B^{2}+v_{0}^{2}\right]+\frac{1}{4 \pi}\left[E^{2}+B^{2}-v^{2}-\frac{3}{2}\left(E^{2}+B^{2}+v_{0}^{2}-v^{2}\right)\right] \\
& =\frac{1}{4 \pi}\left[\frac{1}{2} B^{2}-\frac{1}{2} E^{2}+\frac{1}{2} v^{2}-\frac{1}{2} v_{0}^{2}\right] \\
& =\frac{1}{8 \pi}\left[B^{2}+v^{2}-E^{2}-v_{0}^{2}\right] .
\end{aligned}
$$

95 
b. Force Density Vector

The force density vector may be defined in terms of the divergence of the energy'-momentum tensor. Therefore suppose we calculate the five-dimensional divergence of the tensor $\{T\}$, or

$$
\frac{\partial T}{\partial x^{k}}=\frac{1}{4 \pi} \frac{\partial}{\partial x^{k}}\left[F_{j l} F_{l k}+\frac{1}{4} \delta_{j k} F_{s t} F_{s t}\right] .
$$

Because of the antisymmetry of $F_{j k}$, the first term may be written as

$$
\frac{\partial F_{j \ell}}{\partial x^{k}} F_{l k}=\frac{\partial F_{l j}}{\partial x^{k}} F_{k \ell}
$$

By interchangirg the indices $k$ and $\ell$

$$
\frac{\partial F_{j \ell}}{\partial x^{k}} F_{l k}=\frac{\partial F_{k j}}{\partial x^{l}} F_{l k}=\frac{1}{2}\left(\frac{\partial F_{j \ell}}{\partial x^{k}}+\frac{\partial F_{k \ell}}{\partial x^{\ell}}\right) F_{l k} .
$$

Using the Bianchi identity

$$
\frac{\partial F_{j \ell}}{\partial x^{k}}+\frac{\partial F_{k j}}{\partial x^{\ell}}+\frac{\partial F_{l k}}{\partial x^{j}}=0
$$

the terms contained within the parentheses may be written as

$$
\frac{\partial F_{j \ell}}{\partial x^{k}} F_{l k}=-\frac{1}{2} \frac{\partial F_{l k}}{\partial x^{j}} F_{l k}=-\frac{1}{4} \frac{\partial\left(F_{l k} F_{\ell k}\right)}{\partial x^{i}} .
$$

Substituting this back into the expression for the divergence, the last term will be cancelled because $\ell, k, s$, and $t$ are dumy indices. Then the divergence becomes

$$
\frac{\partial T_{j k}}{\partial x^{k}}=\frac{1}{4 \pi} F_{j \ell} \frac{\partial F_{l k}}{\partial x^{k}}
$$


By interchanging the indices $k$ and $\ell$ on the right-hand side we obtain

$$
\frac{\partial T_{j k}}{\partial x^{k}}=\frac{1}{4 \pi} F_{j k} \frac{\partial F_{k \ell}}{\partial x^{\ell}} \text {. }
$$

The Dynamic force density five-vector may now be defined as

$$
K \equiv \operatorname{Div}_{5}\{T\}
$$

Therefore the components of $K$ are given by

$$
K_{j}=\frac{1}{4 \pi} F_{j k} \frac{\partial F_{k \ell}}{\partial x^{l}} .
$$

But the five-vector current density is given by

$$
\frac{\partial F_{k \ell}}{\partial x^{l}}=\frac{-4 \pi}{c} J_{k}
$$

Thus the components of the five-vector force density become

$$
K_{j}=\frac{-1}{4 \pi} F_{k j}\left(\frac{4 \pi}{c} J_{k}\right)=\frac{-1}{c} J_{k} F_{k j} .
$$

Now, since $\bar{J}_{k}=\left(i c p, \bar{J}, J_{4}\right)$, then

$$
\begin{aligned}
& K_{0}=\frac{i}{c}\left[\bar{J} \cdot \bar{E}+J_{4} V_{0}\right], \\
& \bar{K}=\rho\left[\bar{E}+\frac{1}{c}(\bar{u} \times \bar{B})\right]+\frac{J_{4}}{c} \bar{V},
\end{aligned}
$$

and

$$
K_{4}=\rho V_{0}-\frac{\bar{J} \cdot \bar{V}}{c},
$$


where $\bar{J}=\rho \bar{u}$. These then are the components of the force density five-vector resulting from a gauge field in the Dynamic Theory. These components reduce to the four components of the Lorentz force density should $v_{0}=\bar{\nabla}=0$.

With the interpretation that the four force density components with subscript 1 through 4 are the force density vectors which appear in the first law as $F_{\alpha}$, then the force density vector provides the connection between the first law and the geometry of the sigma manifold discussed in section II.D.2. Thus the existence of the vector field $\phi_{i}$ is also demanded by the Dynamic Theory and need not exist as a separate assumption.

\section{c. Equation of Energy Flow}

Consider the zeroth component of the Dynamic force density five-vector

$$
K_{0}=\frac{\partial T_{0 k}}{\partial x^{k}}=\frac{\partial T_{00}}{\partial x^{0}}+\frac{\partial T_{0 \alpha}}{\partial x^{\alpha}}+\frac{\partial T_{04}}{\partial x^{4}} .
$$

Then

$$
\frac{i}{c}\left[\bar{J} \cdot \bar{E}+J_{4} V_{0}\right]=\frac{\partial \xi}{\partial(i c t)}-\frac{i}{c} \frac{\partial S_{\alpha}}{\partial x^{\alpha}}+\frac{i}{4 \pi} \frac{\partial(\bar{E} \cdot \bar{V})}{\partial x^{4}}
$$

or

$$
\frac{1}{c}\left[\bar{J} \cdot \bar{E}+J_{4} V_{0}\right]=\frac{1}{c} \frac{\partial \xi}{\partial t}+\frac{1}{c} \frac{\partial S_{\alpha}}{\partial x^{\alpha}}-\frac{1}{4 \pi} \frac{\partial(\bar{E} \cdot \bar{V})}{\partial x^{4}}
$$

$$
\begin{aligned}
& \text { or, since } x^{4}=\gamma / a_{0}, \\
& -\bar{E} \cdot \bar{V}-J_{4} V_{0}=\frac{\partial \xi}{\partial t}+\frac{\partial S_{\alpha}}{\partial x^{\alpha}}-\frac{a_{0} c}{4 \pi} \frac{\partial(\bar{E} \cdot \bar{V})}{\partial \gamma} .
\end{aligned}
$$

Rearranging the terms

$$
\operatorname{div} \bar{S}+\frac{\partial \xi}{\partial t}=-\bar{E} \cdot \bar{V}-J_{4} V_{0}+\frac{a_{0} c}{4 \pi} \frac{\partial(\bar{E} \cdot \bar{V})}{\partial \gamma}
$$


and separating out the electrical Poynting vector leads to

$$
\operatorname{div} \bar{S}_{E}+\frac{\partial F}{\partial t}=-\bar{E} \cdot \bar{J}-J_{4} V_{0}-\operatorname{div} \bar{S}_{m}+\frac{{ }^{a}{ }_{0} c}{4 \pi} \frac{\partial(\bar{E} \cdot \bar{V})}{\partial \gamma}
$$

This then is the five-dimensional energy flow equation.

d. Momentum Conservation

The expression for the conservation of momentum may be obtained from the space portion of the force density five-vector

$$
K=\frac{\partial T_{\alpha k}}{\partial x^{k}}=\frac{\partial T_{\alpha 0}}{\partial x^{0}}+\frac{\partial T_{\alpha \beta}}{\partial x^{\beta}}+\frac{\partial T_{\alpha 4}}{\partial x^{4}}, \alpha=1,2,3 .
$$

But $\frac{\partial T_{\alpha \beta}}{\partial x^{B}} \frac{B}{\beta}$ is the three-dimensional divergence of the Dynamic stress tensor $\left\{T^{D}\right\}$, therefore

$$
\bar{K}=-\frac{1}{c^{2}} \frac{\partial \bar{S}}{\partial t}+\operatorname{div}\left\{T^{D}\right\}+\frac{a_{0}}{4 \pi} \frac{\partial}{\partial \gamma}\left[V_{0} \bar{E}+(\bar{V} \times \bar{B})\right] .
$$

If we consider a volume in which all the material is contained and outside of which the field vanishes, then integrating over this volume yields

$$
\int_{v}\left\{\bar{K}+\frac{1}{c^{2}} \frac{\partial \bar{S}}{\partial t}-\frac{a_{0}}{4 \pi} \frac{\partial}{\partial \gamma}\left[V_{0} \bar{E}+(\bar{V} \times \bar{B})\right]\right\} d v=\int_{v} \operatorname{div}\left\{T^{D}\right\} d v
$$

The integral of $\bar{K}$ gives the total force (i.e., the time derivative of the mechanical momentum $\bar{p}$ less the vector $\left(\dot{\gamma}^{\alpha} / \sqrt{1-\beta^{2}}\right)$.

Now define the vector

$$
\bar{g} \equiv \frac{\bar{s}}{c^{2}}-\frac{a_{0}}{4 \pi} \int\left\{\frac{\partial}{\partial \gamma}\left[V_{0} \bar{E}+(\bar{V} \times \bar{B})+\frac{\dot{\gamma} v^{\alpha}}{\sqrt{1-\beta^{2}}}\right\} d t .\right.
$$


Then define

$$
\int_{V} \bar{g} d \sigma \equiv \bar{G}
$$

so that

$$
\frac{d}{d t}(\bar{p}+\bar{G})=\int_{v} \operatorname{div}\left\{T^{D}\right\} d v
$$

Using the divergence theorem the volume integral may be converted to a surface integral so that

$$
\frac{d}{d t}(\bar{p}+\bar{G})=\int_{S}\left\{T^{D}\right\} \cdot \bar{n} d a
$$

If the field vanishes outside of $V$, it must do so also on the boundary surface $s$, hence

$$
\frac{d}{d t}(\bar{p}+\bar{G})=0
$$

Therefore it is not the mechanical momentum $\bar{p}$ but the quantity $\bar{p}+\bar{G}$ which is conserved. Therefore we must interpret $\bar{G}$ as the momentum of the field and

$$
\bar{g}=\frac{\bar{S}}{c^{2}}-\frac{{ }^{a} 0}{4 \pi} \int\left\{\frac{\partial}{\partial \gamma}[V \cdot \bar{E}+(\bar{V} \times \bar{B})]+\frac{4 \pi \dot{\gamma} \bar{v}}{\sqrt{1-\beta^{2}}}\right\} d t
$$

as the momentum density of the field.

e. Gauge Field Pressure

The Dynamic stress tensor is given by

$$
\left.T_{\alpha \beta}^{D}=\frac{1}{4 \pi}\left\{E_{\alpha, \beta} E_{\beta}+B_{\alpha \beta} B_{B}-V_{\alpha \beta} V_{\beta}-\frac{1}{2} \delta_{\alpha \beta} E^{2}+B^{2}+V_{0}^{2}-V^{2}\right]\right\}
$$

100 


$$
=-\frac{1}{8 \pi}\left[E^{2}+B^{2}-v^{2}\right]-\frac{3}{8 \pi} v_{0}^{2} .
$$

Now separate the three-dimensional Dynamic stress tensor into a traceless and an isotropic tensor.

$$
\begin{aligned}
T_{\alpha \beta}^{D}= & \frac{1}{4 \pi}\left\{E_{\alpha \beta} E_{\beta}+B_{\alpha \beta} B_{\beta}-V_{\alpha} V_{\beta}-\frac{1}{2} \delta_{\alpha \beta}\left[E^{2}+B^{2}+V_{0}^{2}-V^{2}\right]\right\} \\
= & \frac{1}{4 \pi}\left\{E_{\alpha} E_{\beta}+B_{\alpha} B_{\beta}-V_{\alpha} V_{\beta}\right\}-\frac{1}{8 \pi} \delta_{\alpha \beta}\left[E^{2}+B^{2}+V_{0}^{2}-V^{2}\right] \\
= & \frac{1}{4 \pi}\left[E_{\alpha} E_{\beta}+B_{\alpha \beta} B_{\beta}-V_{\alpha} V_{\beta}\right]-\left(\frac{2}{3}\right)\left(\frac{1}{8 \pi}\right) \delta_{\alpha \beta}\left[E^{2}+B^{2}-V^{2}\right] \\
& \quad-\left(\frac{1}{3}\right)\left(\frac{1}{8 \pi}\right) \delta_{\alpha \beta}\left[E^{2}+B^{2}+3 V_{0}^{2}-V^{2}\right] \\
\equiv & t_{\alpha \beta}+\tau_{\alpha \beta}^{-}
\end{aligned}
$$

where

$$
t_{c i \beta} \equiv \frac{1}{4 \pi}\left\{E_{\alpha} E_{\beta}+B_{\alpha \beta} B_{\beta}-V_{\alpha} V_{\beta}-\left(\frac{1}{3 \pi}\right) \delta_{\alpha \beta}\left[E^{2}+B^{2}-v^{2}\right]\right.
$$

and

$$
\tau_{\alpha \beta}^{*} \equiv-\left(\frac{1}{24 \pi}\right) \delta_{\alpha \beta}\left[E^{2}+B^{2}+3 v_{0}^{2}-v^{2}\right] .
$$

Now

$$
t_{r}\left\{t_{\alpha \beta}\right\}=\left(\frac{1}{4 \pi}\right)\left[E^{2}+B^{2}-v^{2}-\left(E^{2}+B^{2}-v^{2}\right)\right] \equiv 0
$$

and

$$
t_{r}\left(\tau_{\alpha \beta}^{-}\right\}=-\left(\frac{1}{8 \pi}\right)\left[E^{2}+B^{2}+3 v_{0}^{2}-v^{2}\right] .
$$

101 
Consider the definition

$\frac{1}{3} t \delta_{\alpha \beta} \equiv \tau_{\alpha \beta}^{\prime}=-\left(\frac{1}{24 \pi}\right) \delta_{\alpha \beta}\left[E^{2}+B^{2}+3 v_{0}^{2}-v^{2}\right]$.

Then

$$
t=-\left(\frac{1}{8 \pi}\right)\left[E^{2}+B^{2}+3 v_{0}^{2}-v^{2}\right]
$$

and

$$
\tau_{\alpha \beta}^{\prime}=\left(\begin{array}{ccc}
t & 0 & 0 \\
0 & t & 0 \\
0 & 0 & t
\end{array}\right) .
$$

The isotropic part of the stress tensor is usually called the "pressure." Therefore define

$$
3 p \equiv t
$$

in accordance with customary notation, so that

$$
p=-\left(\frac{1}{24 \pi}\right)\left[E^{2}+B^{2}+3 v_{0}^{2}-v^{2}\right]
$$

With the exception of the factor of 3 this reduces to the "radiation pressure" for an electromagnetic field when $\bar{V}=v_{0}=0$.

Note that this pressure may be zero since it is the sum and difference of squares, or $p=0$, when

$$
V^{2}=E^{2}+B^{2}+3 V_{0}^{2}
$$

This may prove to be an important point when considering boundary conditions in cosmology or the study of elementary particles. 
f. Self Energy of a Charged Particle

In classical electromagnetic theory the self energy of a charged particle is discussed but its value has not been established. This is because the expression for the self energy is a function of the radius associated with the physical extent of the charge distribution. Thus the radius of the charged particle must be known before the self energy can be determined.

Currently the self energy of a charged particle is equated with the energy associated with its inertial mass by

$$
E=m c^{2} \text {. }
$$

Then the radius associated with this energy is taken as the "radius" of the particle. There is no intention that this radius be the physical radius of the particle though it compares favorably with experimental values.

The question arises here of whether or not the Dynamic Theory, with the five-dimensional viewpoint, can theoretically predict the self energy and/or the radius of the physical extent of the mass or charge distribution of the particle.

One of the beneficial aspects of the generalization of physical theory as done in the Dynamic Theory is the possibility of using conceptualizations and procedures developed in one branch of physics in another branch. This aspect of the theory appears applicable here. The self energy of a charged particle is the notion that a certain amount of energy be associated with the existence of the particle and its charge. This notion may be associated with the notion of free energy used in thermodynamics, for, if the self energy of the charged particle is its free energy, then it represents the energy which may be "freed" upon converting the particle into energy. Conversely this would represent the energy required to assemble the charged particle.

With the conceptualization of free energy the second law provides the condition for a stable equilibrium state, namely that a charged particle in an equilibrium state must exist at a minimum of its free energy. Thus if the self energy, or free energy, of a charged particie is sought, then minimizing its free energy will yield the desired result.

Before applying the principle of minimum free energy to a charged particle in a five-dimensional space, suppose that the principle is applied in a fourdimensional manifold first. This procedure will hopefully provide some validity to the method or may point out some difficulty with it. 
The free energy was defined, in analogy with the thermodynamic case, as

$$
G \equiv U-\phi S-x^{\alpha} F_{\alpha},
$$

where $\alpha$ depends upon the applicable work terms which here will be taken as the three spatial dimensions, so that $\alpha=1,2,3$. The first law is given by

$$
\overline{\mathrm{d}} E=\mathrm{d} U-F_{\alpha} \mathrm{dx}{ }^{\alpha}
$$

while the second law yields

$$
\phi \mathrm{d} S=\mathrm{d} U-F_{\alpha} \mathrm{dx}{ }^{\alpha}
$$

for a quasi-static, reversible process. Therefore the differential change in the system energy is

$$
\mathrm{d} U=\phi \mathrm{d} S+F_{\alpha} \mathrm{dx}{ }^{\alpha}
$$

Differentiating Eq. (79) gives the differential change in the free energy as

$$
\mathrm{dG}=\mathrm{d} U-\phi \mathrm{d} S-S \mathrm{~d} \phi-F_{\alpha} \mathrm{dx} \mathrm{x}^{\alpha}-\mathrm{x}^{\alpha} \mathrm{d} F_{\alpha}
$$

Substituting (82) into (83) yields

$$
\mathrm{dG}=-S \mathrm{~d} \phi-\mathrm{x}^{\alpha} \mathrm{d} F_{\alpha}
$$

The force in Eq. (84) is considered to be the Lorentz force

$$
F_{\alpha}=q[\bar{E}+(\bar{v} \times \bar{B})]_{\alpha}
$$

so that Eq. (84) becomes 


$$
\mathrm{dG}=-S \mathrm{~d} \phi-x^{\alpha} \mathrm{d}\left\{\mathrm{q}(\bar{E}+(\bar{v} \times \bar{B}))_{\alpha}\right\}
$$

If we wish to consider the change in free energy with respect to a change in the charge at a constant velocity, we find that, since $\phi$ is a function of velocity only, $d \phi=0$. The specification of constant velocity stems from the desire to obtain the self energy of a charged particle; therefore the particle should be considered as sitting still, so that it will have no kinetic energy. The differential change of free energy for a stationary particle is then

$$
\begin{aligned}
d G & =-S d \phi-x^{\alpha} d\left\{q[\bar{E}+(\bar{v} \times \bar{B})]_{\alpha}\right\} \\
& =-S d \phi-x^{\alpha}\left\{d q[\bar{E}+(\bar{v} \times \bar{B})]_{\alpha}+q d[\bar{E}+(\bar{v} \times \bar{B})]_{\alpha}\right\},
\end{aligned}
$$

so that for $\phi=$ constant

$$
\left(\frac{\partial G}{\partial q}\right)_{\phi}=-x^{\alpha}[\bar{E}+(\bar{v} \times \bar{B})]_{\alpha}-x^{\alpha} q\left(\frac{\partial[\bar{E}+(\bar{v} \times \bar{B})]_{\alpha}}{\partial q}\right)_{\phi} .
$$

But $\bar{E}+(\bar{v} \times \bar{B})$ is independent of the charge $q$ and therefore

$$
\left(\frac{\partial G}{\partial q}\right)_{\phi}=-x^{\alpha}[\bar{E}+(\bar{v} \times \bar{B})]_{\alpha}
$$

If the charge is not in motion, then

$$
\left(\frac{\partial G}{\partial q}\right)_{\phi}=-x^{\alpha} E_{\alpha}
$$

since $\bar{v}=0$. Further, if the field $\vec{E}$ is due to the charged particle, then radial symmetry yields

$$
\bar{E}=\frac{-\mathrm{e} \hat{r}}{4 \pi \varepsilon_{0} r^{2}}
$$


for points external to the negative charge distribution. Substituting (89) into (88) results in

$$
\left(\frac{\partial G}{\partial q}\right)_{\phi}=\frac{r e}{4 \pi \varepsilon_{0} r^{2}}=\frac{\epsilon}{4 \pi \varepsilon_{0}} \bar{r}
$$

Thus we may integrate over the charge $q$ at the radius $R$ to get

$$
\int_{G_{0}}^{G} d G=\frac{e}{\cdot \pi \varepsilon_{0}^{R}} \int_{0}^{-e} d q,
$$

so that the free energy is given by

$$
G=\frac{-e^{2}}{4 \pi \varepsilon_{0} R}+G_{0}
$$

Here the use of the (-e) in the limits of integration corresponds to the notion of free energy as the energy required to bring the charge to the radius $R$.

Thus the free energy given by' Eq. (91), and hence the adopted method, differs from the classical expression for the self energy of a crarged particle by the constant $G_{0}$ oniy. From Eq. (91) it may be seen that the value for the free energy depends upon the physical radius $R$ by an inverse relation. This functional relationship obviously will not yield a finite value of $R$ that minimizes the free energy. Therefore we are no better off than we were by using the classical theory.

The question of interest though is whether or not the five-dimensional viewpoint will shed a new light on the self energy. By the preceding development's arriving at an expression consistent with the classical self energy of a charged particle, a measure of validity has been obtained for using the method.

Suppose now we apply the freceding procedure from a five-dimensional point of view. In a five-dimensional system the forces are given by

$$
F_{\alpha}=\mathrm{q}[\bar{E}+(\overline{\mathrm{v}} \times \overline{\mathrm{B}})]_{\alpha}+\frac{\mathrm{J}_{4}}{\mathrm{c}} \overline{\mathrm{v}}_{\alpha}, \alpha=1,2,3,
$$


and

$$
F_{4}=\mathrm{q}\left[\mathrm{V}_{0}-\frac{\bar{v} \cdot \overline{\mathrm{V}}}{\mathrm{c}}\right]
$$

Thus

$$
\begin{aligned}
x^{\alpha} d F_{\alpha} & =x^{\alpha} d\left[q[\bar{E}+(\bar{v} \times \bar{B})]_{\alpha}+\frac{J_{4} v_{\alpha}}{c}\right\}+x^{4} d\left[q\left[v_{0}-\frac{\bar{v} \cdot \bar{v}}{c}\right]\right\} \\
& =x^{\alpha} d q[\bar{E}+(\bar{v} \times \bar{B})]_{\alpha}+x^{\alpha} \frac{d\left(J_{4} v_{\alpha}\right)}{c} \\
& +x^{\alpha} q d[\bar{E}+(\bar{v} \times \bar{B})]_{\alpha}+x^{4} d q\left[v_{0}-\frac{\bar{v} \cdot \bar{v}}{c}\right]+x^{4} q d\left[v_{0}-\frac{\bar{v} \cdot \bar{v}}{c}\right] .
\end{aligned}
$$

Then for a stationary particle and charge so that $\bar{v}=0$,

$$
\left(\frac{\partial G}{\partial q}\right)_{\phi}=-x^{\alpha} E_{\alpha}-x^{4} v_{0}-\frac{x^{\alpha}}{c}\left\{\left(\frac{\partial\left(J_{4} V_{\alpha}\right)}{\partial q}\right)_{\phi}+c q\left(\frac{\partial E_{\alpha}}{\partial q}\right)_{\phi}\right\}-x^{4} q\left(\frac{\partial v_{0}}{\partial q}\right)_{\phi} .
$$

Again suppose that the electric field is due to a spherically symmetric charge distribution so that

$$
\bar{E}=-\frac{e \hat{r}}{4 \pi \varepsilon r_{0}^{2}}
$$

which is independent of $q$. Thus Eq. (94) becomes

$$
\left(\frac{\partial G}{\partial q}\right)_{\phi}=\frac{e}{4 \pi \varepsilon_{0} r}-x^{4} v_{0}-\frac{x^{\alpha}}{c}\left(\frac{\partial J_{4} V_{\alpha}}{\partial q}\right)_{\phi}-x^{4} q\left(\frac{\partial v_{0}}{\partial q}\right)_{\phi} .
$$

It is not obvious whether or not $\mathrm{J}_{4}, \bar{V}$ and $\mathrm{V}_{0}$ are independent of $q$ when the seven Maxwe11 equations are first considered. However, with the tentative interpretation that these quantities are related with nuclear forces we can suppose 
that they are independent of the charge $q$ and

$$
\left(\frac{\partial G}{\partial q}\right)_{\phi}=\frac{e}{4 \pi \varepsilon_{0} r}-x^{4} v_{0}
$$

Since $x^{4}=\gamma / a_{0}$ Eq. (96) becomes

$$
\left(\frac{\partial G}{\partial q}\right)_{\phi}=\frac{e}{4 \pi \varepsilon_{0} r}-\frac{\gamma}{a_{0}} v_{0}
$$

where $\gamma$ is the mass density of the particle. Integrating Eq. (97) at the radius $\mathrm{R}$

$$
\int_{G_{0}}^{G} d G=\frac{e}{4 \pi \varepsilon_{0} R} \int_{0}^{-e} d q-\int_{0}^{-e} \frac{\gamma v_{0}}{a_{0}} d q
$$

or

$$
G=\frac{-e^{2}}{4 \pi \varepsilon_{0} R}-\frac{v_{0}}{a_{0}} \int_{0}^{-e} \gamma d q,
$$

since $V_{0}$ was assumed to be independent of $q$.

The charge $q$ is external to the charged particle and therefore should be independent of the mass $d_{t}-i$ ty so that the free energy becomes

$$
G=\frac{-e^{2}}{4 \pi \varepsilon_{0} R}+\frac{e \gamma V_{0}}{a_{0}}+G_{0} .
$$

For a charged particle the mass density will depend upon the physical extent of the particle and hence upon $R$. The field quantity $V_{0}$ is supposed to be related to nuclear field properties and may also depend upon $R$. Thus there should be a finite radius of physical extent $R$ which will minimize the free energy given by Eq. (99). 
If the functional dependence of $V_{0}$ and $\gamma$ upon the radius $R$ were known, then a minimum of the free energy would be given by determining the radius $R_{0}$ for which

$$
\left(\frac{\partial G}{\partial R}\right)_{R_{0}}=0=\frac{+e^{2}}{4 \pi \varepsilon_{0} R^{2}}+\frac{e}{a_{0}} \frac{\partial}{\partial R}\left(\gamma V_{0}\right) .
$$

Before Eq. (100) may be used to find the radius $R_{0}$ which minimizes the free energy, an appeal to the seven Maxwell equations and/or a model of the particle must be made so that the dependence of the product $\gamma_{0}$ upon $R$ can be determined.

Suppose a simple model of the particle is made in order to further investigate the existence of a finite radius $R_{0}$ which will minimize the free energy. If a uniform mass distribution in a spherical shape is considered, then

$$
\gamma=\frac{m}{\frac{4}{3} \pi R^{3}}
$$

and Eq. (99) becomes

$$
G=\frac{-e^{2}}{4 \pi \varepsilon_{0}^{R}}+\frac{3 e m V_{0}}{4 \pi R^{3} a_{0}}+G_{0}
$$

If it is also supposed that $V_{0}$ has a $(1 / R)$ dependence, then

$$
G=\frac{-e^{2}}{4 \pi \varepsilon_{0} R}+\frac{3 e m k}{4 \pi a R^{R^{4}}}+G_{0} \text {. }
$$

Differentiating Eq. (102) with respect to $R$ yields

$$
\left(\frac{\partial G}{\partial R}\right)_{R_{0}}=0=\frac{e^{2}}{4 \pi \varepsilon_{0} R^{2}}-\frac{12 e m k}{4 \pi a a_{0} R^{5}}
$$


or

$$
\frac{e}{\varepsilon_{0}}=\frac{12 m k}{a_{0} R_{0}^{3}}
$$

Then $R_{0}=3 \sqrt{\left(12 m k \varepsilon_{0}\right) /\left(e a_{0}\right)}$ is the radius which would minimize the free energy for this model.

The minimum value of the free energy would then be given by

$$
\begin{aligned}
G_{\min } & =\frac{e^{2}}{4 \pi \varepsilon_{0} R_{0}}\left\{\frac{3 m k \varepsilon_{0}}{e a_{0} R_{0}^{3}}-1\right\}+G_{0} \\
& =\left(\frac{e^{2}}{4 \pi \varepsilon_{0}}\right) 3 \sqrt{\frac{{ }^{e a_{0}}}{12 m \varepsilon_{0} k}}\left\{\frac{3 m \varepsilon_{0} k}{e_{0}}\left(\frac{e_{0}}{12 m \varepsilon_{0} k}\right)-1\right\}+G_{0} \\
& =\left(\frac{e^{2}}{4 \pi \varepsilon_{0}}\right) 3 \sqrt{\frac{e a_{0}}{12 m \varepsilon_{0} k}}\left\{-\frac{1}{4}\right\}+G_{0},
\end{aligned}
$$

or

$$
G_{\min }=G_{0}-\frac{e^{2}}{16 \pi \varepsilon_{0} R_{0}} .
$$

Though the self energy given by Eq. (104) is based upon an unverified model, it does represent a demonstration that the Dynamic Theory can produce a predicted value of self energy for a charged particle. It remains then to determine a realistic model of the particle or another method of determining the functional dependence of $Y_{0}$ upon the radius $R$ plus determining the value of the universal constant $a_{0}$. Once this has been done, then the self energy, or free energy, may be specified. 


\section{c. Quantization in Five Dimensions}

The preceding development provides a tremendous wealth of mathematical abstractions. However, there seems within it no readily apparent methed of interpreting the new fields. If there appears to be no physical entity which may be associated with the new field quantities, then the development will have gone for naught. On the other hand, with the notion of nuclear fields in mind it seems that if the new field quantities are included in a quantized picture, then perhaps the relation to nuclear fields may be made.

In the following the requirement for quantization is provided by appropriate restrictions upon a system whose description is taken from the Dynamic Theory. However, the use of the five-dimensional Dirac equation has not yet been shown to result from the Dynamic Theory. Schrodinger's quantum mechanics may be obtained using London's work, but I am not aware of a procedure to arrive logically at Dirac's equation even though 1 feel that the method exists. As it now stands, the use of the generalized Dirac equation must be accepted as an independent fundamental assumption.

1. Quantization. The system under consideration now is a five-dimensional system with arc element

$$
\left(d q^{0}\right)^{2}=f(d \sigma)^{2}
$$

Now since our system is an $E$-conservative, $\overline{\mathrm{d}} E=0$, system the principle of increasing entropy requires that $\left(d q^{0}\right)^{2} \geq 0$ so that $f(d \sigma)^{2} \geq 0$. Introducing the quantization conditions results in

$$
\int \phi_{j} d x^{j}=2 \pi i n, j=0,1,2,3,4 \text {, }
$$

where $\phi_{j} \equiv \pm \frac{\partial \operatorname{lnf} f^{1 / 2}}{\partial x^{j}}$ and $x^{0} \equiv c t, x^{1} \equiv q^{1}, x^{2} \equiv q^{2}, x^{3} \equiv q^{3}, x^{4} \equiv \frac{\gamma}{a_{0}}$.

If we restrict ourselves to a $(d \sigma)^{2}$ space which is the local Euclidean space, then $(d \sigma)^{2}$ is the five-dimensional Minkowski-type manifold; using London's work we would produce a five-dimensional quantum dynamical system. 
2. Five-Dimensional Hamiltonian. We previously showed that the principle of increasing entropy resulted in

$$
\delta \int \gamma \sqrt{\left(d q^{0}\right)^{2}}=0
$$

as the variational principle for a local Euclidean manifold. Since multiplication by a constant does not change the problem we may take our variational problem to be

$$
\delta \int \gamma c^{2} \sqrt{\left(d q^{0}\right)^{2}}=0
$$

Defining the velocity vector as $u^{j} \equiv \frac{d x^{j}}{d q^{0}}$ and the momentum as $p_{j} \equiv \frac{\partial L}{\partial u^{j}} \equiv \gamma g_{j k} u^{k}$, where we have used the fact that $g_{j k} u^{j} u^{k}=1$, then we may form the contravariant momentum as

$$
p^{j}=g^{j k} p_{k}=g^{j k} \gamma g_{k \ell} u^{\ell},
$$

so that

$$
\begin{aligned}
p_{j} p^{j} & =\left(\gamma g_{j \ell} u^{j}\right)\left(g^{j k} \gamma g_{k \ell} u^{\ell}\right)=\gamma^{2} \delta_{j \ell} u^{\ell} g_{j k} u^{k} \\
& =\gamma\left(\gamma g_{i k} u^{j} u^{k}\right) \\
& =\gamma^{2} c^{2},
\end{aligned}
$$

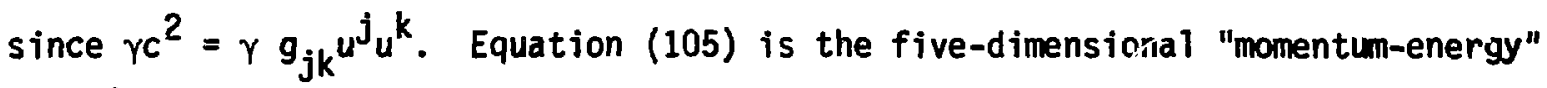
equation.

He may now follow London's procedure to obtain our wave function for the five-dimensional system. However, a quicker way to investigate the effect of the Dynamic Theory upon quantum mechanics would seem to be that of adopting 
Dirac's equation in a five-dimensional form and following a development analogous to standard four-dimensional relativistic quantum mechanics. With this in mind, then we shall adopt the form

$$
h=i\left(\tilde{\alpha}_{1} \frac{\partial}{\partial x^{1}}+\tilde{\alpha}_{2} \frac{\partial}{\partial x^{2}}+\tilde{\alpha}_{3} \frac{\partial}{\partial x^{3}}+\tilde{\alpha}_{4} \frac{\partial}{\partial x^{4}}\right)-\tilde{\beta}
$$

to be the five-dimensional specific Hamiltonian operator. The partial derivative operators are specific operators and hence are dimensionless in natural units. In Eq. (106) the $\tilde{\alpha}$ 's and $\tilde{\beta}$ do not involve derivatives and must be Hermitian in order that $h$ be Hermitian.

By taking the four partial derivatives in Eq. (106) as the four-vector specific momentum operator we may write

$$
h=-(\tilde{\alpha} \cdot \bar{p}+\tilde{\beta}) .
$$

3. Five-Dimensional Dirac Equation. If we take $p^{0}|>=h|>$ and require that the $\tilde{\alpha}^{\prime} s$ and $\tilde{\beta}$ are chosen such that solutions of this equation are also solutions of Eq. (107), we find the restrictions imposed upon the choice of the $\tilde{\alpha}^{\prime} s$ and $\tilde{B}$ to be:

$$
\begin{aligned}
(\overline{\tilde{\alpha}} \cdot \bar{p})^{2} & =p^{2}, \\
\tilde{\beta}^{2} & =1,
\end{aligned}
$$

and

$$
\overline{\alpha \bar{\beta}}+\overline{\tilde{\beta} \tilde{\alpha}}=0,
$$

where natural units, $c=1$, are used.

A set of $8 \times 8$ matrices satisfying the requirements of Eq. (108) is

$$
\tilde{\beta}=\left(\begin{array}{ll}
\beta & 0 \\
0 & \beta
\end{array}\right), \quad \tilde{\alpha}_{i}=\left(\begin{array}{cc}
0 & \alpha_{i} \\
\alpha_{i} & 0
\end{array}\right) i=1,2,3, \tilde{\alpha}_{4}=\left(\begin{array}{ll}
A & 0 \\
0 & A
\end{array}\right) \text {, }
$$


where

$$
\beta=\left(\begin{array}{cc}
I & 0 \\
0 & -I
\end{array}\right), \quad \alpha_{i}=\left(\begin{array}{cc}
0 & \sigma_{i} \\
\sigma_{i} & 0
\end{array}\right) i=1,2,3, \text { and } A=\left(\begin{array}{ll}
0 & I \\
I & 0
\end{array}\right)
$$

and

$$
I=\left(\begin{array}{ll}
1 & 0 \\
0 & 1
\end{array}\right), \sigma_{1}=\left(\begin{array}{ll}
0 & 1 \\
1 & 0
\end{array}\right), \sigma_{2}=\left(\begin{array}{cc}
0 & -i \\
i & 0
\end{array}\right) \text {, and } \sigma_{3}=\left(\begin{array}{cc}
1 & 0 \\
0 & -1
\end{array}\right) \text {. }
$$

Then the five-dimensional Dirac equation may be taken to be

$$
i \frac{\partial}{\partial t} \Psi(x)=(i \tilde{\alpha} \cdot \nabla-\tilde{\beta}) \Psi(x),
$$

where the $\nabla$ is a four-dimensional operator. By defining

$$
\gamma^{0} \equiv \tilde{\beta} ; \quad \gamma^{\mu} \equiv-\tilde{\beta} \tilde{\alpha}_{\mu}(\mu=1,2,3,4),
$$

then Eq. (110) may be written as

$$
\left(i \partial_{j} \gamma^{j}+1\right) \Psi(x)=0 \text {. }
$$

By virtue of the properties of the $\tilde{\alpha}^{\prime} s$ and $\tilde{\beta}$ plus the fact that

$$
\mathbf{g}^{j k}=\left\{\begin{array}{l}
1 \text { for } j=k=0 \\
-1 \text { for } j=k=1,2,3,4, \\
0 \text { for } j \neq k
\end{array}\right.
$$

the anticommutator of the $\gamma$-matrices must satisfy

$$
\left\{\gamma^{j}, \gamma^{i}\right\}=2 g^{j i}
$$


4. "Lorentz" Covariance. Under a five-dimensional Lorentz transformation

$$
x^{j-}=L_{k}^{j} x^{k}
$$

we shall suppose each component of the wave function $\psi(x)$ transforms into a linear combination of all four components:

$$
\Psi(x) \overrightarrow{\mathrm{LT}} \Psi^{-}\left(\mathrm{x}^{-}\right)=S \Psi(\mathrm{x}),
$$

where $S$ is a Dirac spinor satisfying

$$
s^{-1} \gamma^{j} s=L_{k}^{j} \gamma^{k}
$$

By using an infinitesimal Lorentz transformation given by

$$
L_{k}^{j}=g_{k}^{j}+d \theta \varepsilon_{k}^{j}
$$

where $\varepsilon_{k}^{j}$ are a set of 16 numbers, then $S(\theta)$ may be shown to be given by

$$
S(\theta)=\exp \left(T \int_{0}^{\theta} \mathrm{d} \theta\right),
$$

where the matrix $T$ is given by

$$
T=\frac{1}{4} \varepsilon_{j k} \gamma^{j} \gamma^{k}
$$

Equations (113), (114), and (115) suffice to guarantee the Lorentz covariance of the five-dimensional Dirac equation.

Following standard quantum mechanical procedure we shall adopt the proba- bility current density to be

$$
j^{k}(x)=\bar{\Psi}(x) \gamma^{k} \Psi(x)
$$


with the requirements that

$$
\begin{aligned}
& \partial_{k} j^{j}=0, \\
& j^{k} \text { transforms as a contravariant vector, and } \\
& j^{k} \text { must be real . }
\end{aligned}
$$

5. Spin. In the three-dimensional space the angular momentum is given by the vector $L$ as the cross product of the coordinates and momenta. We shall then define the angular four-momentum to be the four-dimensional cross product

$$
\bar{L} \equiv \varepsilon_{\mathbf{i j k}} \mathbf{x}^{\mathbf{j}} \mathbf{p}^{\mathbf{k}}
$$

where $x^{4}$ is the mass density and

$$
\varepsilon_{i j k} \equiv\left\{\begin{array}{c}
0 \text { if any two indices are alike } \\
1 \text { for even permutation to align indices in } \\
\text { ascending order } \\
-1 \text { for odd permutation to align indices in } \\
\text { ascending order }
\end{array}\right.
$$

Then the commutator of the components of the angular four-momentum with the specific Hamiltonian is not zero; for instance

$$
\left[L_{3}, \hat{h}\right]=i \gamma^{0} \gamma^{1} p^{2}-i \gamma^{0} \gamma^{2} p^{1}+i \gamma^{0} \gamma^{4} p^{1}-i \gamma^{0} \gamma^{1} p^{4}+i \gamma^{0} \gamma^{4} p^{2}-i \gamma^{0} \gamma^{2} p^{4} .
$$

Now suppose there exists a four-spin vector $\bar{S}$ such that the sum of the angular four-momentum and the four-spin vector commutes with the specific Hamiltonian; then if we define a new three-spin vector $\bar{u}$ given by the components $u_{1} \equiv \frac{1}{2} i \gamma^{4} \gamma^{1}, u_{2} \equiv \frac{1}{2} i \gamma^{4} \gamma^{2}$, and $u_{3} \equiv \frac{1}{2} i \gamma^{4} \gamma^{3}$, and take the usual spin vector $\bar{s}$ given by $s_{1}=\frac{1}{2} i \gamma^{2} \gamma^{3}, s_{2}=\frac{1}{2} i \gamma^{3} \gamma^{1}$, and $s_{3}=\frac{1}{2} i \gamma^{1} \gamma^{2}$, the components of the four-spin vector may be shown to be 


$$
\begin{aligned}
& s_{1}=s_{1}-u_{2}-u_{3}, \\
& s_{2}=s_{2}+u_{1}-u_{3}, \\
& s_{3}=s_{3}+u_{1}+u_{2},
\end{aligned}
$$

and

$$
\mathfrak{s}_{4}=s_{1}-s_{2}+s_{3}
$$

In analogy with standard relativistic quantum mechanics the eigenvalues of the four-spin components can be shown to be $\pm \sqrt{3 / 4}$. It may also be shown that the set of observables $\bar{P}, h$, and $\bar{S} \cdot \bar{P}$, where $\bar{P}$ is the four-momentum and $\bar{S}$ is the four-spin, form a complete set of cormuting observables.

6. Dirac Equation with Fields. In analogy with relativistic quantum mechanics we take the five-dimensional Dirac equation to be

$$
\left[\left(i \partial_{j}-\phi_{j}\right) \gamma^{j}+1\right] \Psi=0
$$

where $\phi_{j}$ is five-vector potential. By operating on the left with $\left[\left(i \partial_{j}-\phi_{j}\right) \gamma^{j}\right.$ - 1] and separating $\gamma^{j} \gamma^{k}$ into symmetric and antisymmetric parts as

$$
\gamma^{i} \gamma^{k}=\frac{1}{2}\left\{\gamma^{i}, \gamma^{k}\right\}+\frac{1}{2}\left[\gamma^{j}, \gamma^{k}\right] \equiv g^{i k}+\sigma^{j k},
$$

then Eq. (116) becomes

$$
\left[\left(i \partial_{j}-\phi_{j}\right)\left(i \partial^{j}-\phi^{j}\right)-1+\left(-\partial_{j} \partial_{k}+\phi_{j} \phi_{k}-i \phi_{j} \partial_{k}-i \partial_{j} \phi_{k}\right) \sigma^{j k}\right] \Psi=0
$$

Separating $\partial_{j} \phi_{k}$ into symmetric and antisymmetric parts as

$$
\partial_{j} \phi_{k}=\frac{1}{2}\left(\partial_{j} \phi_{k}+\partial_{k} \phi_{j}\right)+\frac{1}{2}\left(\partial_{j} \phi_{k}-\partial_{k} \phi_{j}\right)
$$


and defining the field tensor as

$$
F_{j k}=\partial_{j} \phi_{k}-\partial_{k} \phi_{j},
$$

Eq. (118) becomes

$$
\left[\left(i \partial_{j}-\phi_{j}\right)\left(i \partial^{k}-\phi^{k}\right)-1-\frac{1}{2} i F_{j k} 0^{j k}\right] \Psi=0 .
$$

Now since

$$
\sigma^{j k}=\mid \begin{gathered}
0 \\
-\dot{x}^{1} \\
-\dot{x}^{2} \\
-\dot{x}^{3} \\
-\dot{x}^{4}
\end{gathered}
$$

$\dot{x}^{1}$
0
$2 i s^{3}$
$-2 i s^{2}$
$-n^{1}$

$\dot{x}^{2}$
$-2 i s^{3}$
0
$2 i s^{1}$
$-n^{2}$
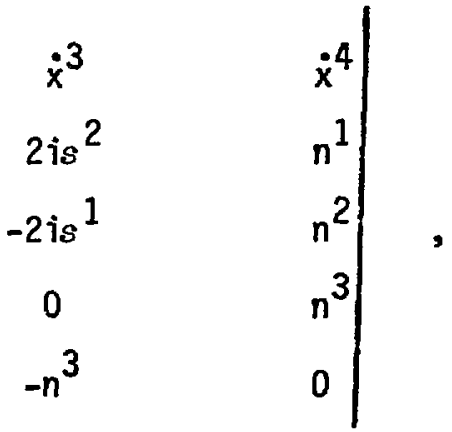

where $\sigma^{j_{4}} \equiv n^{j}$ for $j=1,2,3$, and
$F_{j k}=\mid \begin{gathered}0 \\ -E_{1} \\ -E_{2} \\ E_{3} \\ -V_{0}\end{gathered}$
$E_{1}$
$E_{2}$
$E_{3}$
$v_{0}$
$-\mathrm{B}_{3}$
$\mathrm{B}_{2}$
$v_{1}$
0
$-\mathrm{B}_{1}$
$v_{2}$
$\mathrm{B}_{1}$
0
$v_{3}$
$-v_{1}$
$-V_{2}$
0

plus recalling the seven Maxwell-type equations

$$
\begin{aligned}
& \bar{\nabla} \cdot \bar{B}=0, \quad \bar{\nabla} \times \bar{E}+\frac{1}{c} \frac{\partial \bar{B}}{\partial t}=0, \quad \bar{\nabla} \cdot \bar{E}=4 \pi p-a_{0} \frac{\partial V_{0}}{\partial \gamma}, \\
& \bar{\nabla} \times \bar{B}-\frac{1}{c} \frac{\partial \bar{E}}{\partial t}=\frac{4 \pi \bar{J}}{c}-a_{0} \frac{\partial \bar{V}}{\partial \gamma}, \quad \bar{\nabla} \times \bar{V}+a_{0} \frac{\partial \bar{B}}{\partial \gamma}=0,
\end{aligned}
$$




$$
\bar{\nabla} V_{0}+\frac{1}{c} \frac{\partial \bar{V}}{\partial t}+a_{0} \frac{\partial \bar{E}}{\partial \gamma}=0, \quad \bar{\nabla} \cdot \bar{V}+\frac{1}{c} \frac{\partial V_{0}}{\partial t}=-\frac{4 \pi}{c} J_{4},
$$

then Eq. (119) may be written as

$$
\left[\left(i \partial_{j}-\phi_{j}\right)\left(i \partial^{k}-\phi^{k}\right)-1+2 \bar{B} \cdot \bar{s}-i \bar{E} \cdot \dot{\bar{x}}-i v_{0} \dot{x}^{4}-i n \cdot v\right] \Psi=0
$$

and thus becomes the Dirac equation with fields $\bar{E}, \bar{B}, V_{0}$, and $\bar{V}$.

Suppose we consider a system without an electric charge so that $p=\bar{J}=0$, then by Eq. (120) we still have

$$
\bar{\nabla} \cdot \bar{E}=-a_{0} \frac{\partial V_{0}}{\partial \gamma} \quad \text { and } \quad \bar{\nabla} \times \bar{B}-\frac{1}{c} \frac{\partial \bar{E}}{\partial t}=-a_{0} \frac{\partial \bar{V}}{\partial \gamma}
$$

and therefore there will still be a magnetic moment.

This then sets up an argument which may provide an interpretation of the new field quantities. Suppose that an electron, because of its small amount of mass when compared to a proton or neutron, does not involve sufficient mass density change to warrant using the fifth dimension. Then the magnetic moment of the electron should be given accurately by the relativistic Dirac equation.

But suppose that nucleons (i.e., protons and neutrons) have sufficient mass density change to warrant using the fifth dimension protided by the dynamic theory. Then the nucleons involve the new field components and we should expect a different value for the magnetic moment of a proton. For the neutron, which has no electric charge, we find a magnetic moment due to these riew field components.

Now if we assume that the difference between the observed values of the magnetic moments of the proton and the neutron and the predicted values of relativistic quantum theory are due to the strong interaction, or nuclear forces, then we must connect the new field quantities with the nuclear field quantities.

7. Allowed Fundamental Spin States. In the five-dimensional quantization of the space-time-mass manifold three spin vectors appear. One of these is the familiar three-component spin vector of relativistic quantum mechanics. The second of the three is a new three-component spin vector while the remaining one is a four-component spin vector. 
Using the theorem:

If $\alpha$ satisfies $\alpha^{2}=a^{2}$ where $a$ is a number, then the eigenvalues of $\alpha$ are \pm a.

Then it is not difficult to show that the component eigenvalues are

$$
s_{\alpha}= \pm \frac{1}{2}, u_{\alpha}= \pm \frac{1}{2}, s_{j}^{2}=\frac{3}{4}, \alpha=1,2,3 \text { and } j=1,2,3,4 \text {. }
$$

If, in analogy with the eigenvalues for the total angular momentum, we write

$$
s_{j}^{2}=\frac{3}{4}=s_{j}\left(s_{j}+1\right)
$$

then the possible eigenvalues becomes

$$
s_{\alpha}= \pm \frac{i}{2}, u_{\alpha}= \pm \frac{1}{2}, s_{j}=\frac{1}{2},-\frac{3}{2}
$$

However, the following relations restrict the number of possible combinations of these eigenvalues.

$$
\begin{aligned}
& \mathrm{s}_{1}=s_{1}-u_{2}-u_{3}, \\
& \mathrm{~s}_{2}=s_{2}+u_{1}-u_{3}, \\
& \mathrm{~s}_{3}=s_{3}+u_{1}+u_{2},
\end{aligned}
$$

and

$$
\mathrm{S}_{4}=s_{1}-s_{2}+s_{3}
$$

The question asked now is, how many combinations of the above eigenvalues are allowed? 
For $s_{1}=\frac{1}{2}$ the combination $s_{1}=-\frac{1}{2}$ and $u_{2}=\frac{1}{2}$ is impossible.

For $s_{1}=\frac{1}{2}$ the combination $s_{2}=-\frac{1}{2}$ and $u_{1}=-\frac{1}{2}$ is impossible.

For $s_{3}=\frac{1}{2}$ the combination $s_{3}=-\frac{1}{2}$ and $u_{1}=-\frac{1}{2}$ is impossible.

For $S_{4}=\frac{1}{2}$ the combination $s_{1}=-\frac{1}{2}$ and $s_{2}=\frac{1}{2}$ is impossible.

For $s_{1}=-\frac{3}{2}$ only one combination is possible: $s_{1}=-\frac{1}{2}, u_{2}=\frac{1}{2}$, and $u_{3}=\frac{1}{2}$.

For $s_{2}=-\frac{3}{2}$ only one combination is possible: $s_{2}=-\frac{1}{2}, u_{1}=-\frac{1}{2}$, and $u_{3}=\frac{1}{2}$.

For $s_{3}=-\frac{3}{2}$ only one combination is possible: $s_{3}=-\frac{1}{2}, u_{1}=-\frac{1}{2}$, and $u_{2}=-\frac{1}{2}$.

For $S_{4}=-\frac{3}{2}$ only one combination is possible: $s_{1}=-\frac{1}{2}, s_{2}=\frac{1}{2}$, and $s_{3}=-\frac{1}{2}$.

Now because $\mathrm{S}_{4}$ is a combination of the first terms of each of the components $s_{1}, s_{2}$, and $s_{3}$, not all of the above listed 16 combinations are possible.

For $s_{4}=\frac{1}{2}$ the following combinations of $\left(s_{1}, s_{2}, s_{3} ; u_{1}, u_{2}, u_{3}\right)$ are possible.

(1) $\left(\frac{1}{2}, \frac{1}{2}, \frac{1}{2} ;-\frac{1}{2}, \frac{1}{2},-\frac{1}{2}\right) \quad$ for $s_{1}=s_{2}=s_{3}=\frac{1}{2}$

(2) $\left(\frac{1}{2}, \frac{1}{2}, \frac{1}{2} ; \frac{1}{2},-\frac{1}{2}, \frac{1}{2}\right) \quad$ for $s_{1}=s_{2}=s_{3}=\frac{1}{2}$

(3) $\left(\frac{1}{2},-\frac{1}{2},-\frac{1}{2} ; \frac{1}{2}, \frac{1}{2},-\frac{1}{2}\right)$ for $s_{1}=s_{2}=s_{3}=\frac{1}{2}$

(4) $\left(-\frac{1}{2},-\frac{1}{2}, \frac{1}{2} ; \frac{1}{2},-\frac{1}{2},-\frac{1}{2}\right)$ for $s_{1}=s_{2}=s_{3}=\frac{1}{2}$.

121 
The remaining combinations are:
(5) $\left(-\frac{1}{2}, \frac{1}{2},-\frac{1}{2} ;-\frac{1}{2},-\frac{1}{2},-\frac{1}{2}\right)$ for $S_{4}=S_{3}=-\frac{3}{2} ; S_{1}=S_{2}=\frac{1}{2}$
(6) $\left(-\frac{1}{2}, \frac{1}{2},-\frac{1}{2} ; \frac{1}{2}, \frac{1}{2}, \frac{1}{2}\right)$
for $s_{4}=s_{1}=-\frac{3}{2} ; S_{2}=S_{3}=\frac{1}{2}$
(7) $\left(\frac{1}{2},-\frac{1}{2},-\frac{1}{2} ;-\frac{1}{2},-\frac{1}{2}, \frac{1}{2}\right)$
for $s_{2}=s_{3}=-\frac{3}{2} ; s_{1}=s_{4}=\frac{1}{2}$
(8) $\left(-\frac{1}{2},-\frac{1}{2}, \frac{1}{2} ;-\frac{1}{2}, \frac{1}{2}, \frac{1}{2}\right)$
for $s_{1}=s_{3}=-\frac{3}{2} ; s_{2}=s_{4}=\frac{1}{2}$.

Thus there is an octet of possible combinations. There are also some obvious symmetries in these combinations. An aid in seeing these symmetries is the vector defined as $\bar{t}$ where

$$
t_{1} \equiv-\left(u_{2}+u_{3}\right) ; t_{2} \equiv u_{1}-u_{3} ; t_{3} \equiv u_{2}+u_{3}
$$

Then for each of the eight combinations above we find $\left(t_{1}, t_{2}, t_{3}\right)$ given by
(1) $\bar{t}=(0,0,0)$
(5) $\overline{\mathrm{t}}=(1,0,-1)$
(2) $\bar{t}=(0,0,0)$
(6) $\bar{t}=(-1,0,1)$
(3) $\bar{t}=(0,1,1)$
(7) $\bar{t}=(0,-1,-1)$
(4) $\bar{t}=(1,1,0)$
(8) $\bar{t}=(-1,-1,0)$

Thus the eight combinations correspond to four distinct $\bar{t}$ vectors which carry a \pm sign. Or

$$
\bar{t}_{1}=(0,0,0) ; \bar{t}_{2}=(0,1,1) ; \bar{t}_{3}=(1,1,0) ; \bar{t}_{4}=(1,0,-1)
$$

For $+\bar{t}_{\alpha}$ we have:

$$
t_{1} \Rightarrow(\bar{s} ; \bar{u})=\left(\frac{1}{2}, \frac{1}{2}, \frac{1}{2} ;-\frac{1}{2}, \frac{1}{2},-\frac{1}{2}\right)
$$




$$
\begin{aligned}
& t_{2} \Rightarrow(\bar{s} ; \bar{u})=\left(\frac{1}{2},-\frac{1}{2},-\frac{1}{2} ; \frac{1}{2}, \frac{1}{2},-\frac{1}{2}\right) \\
& t_{3} \Rightarrow(\bar{s} ; \bar{u})=\left(-\frac{1}{2},-\frac{1}{2}, \frac{1}{2} ; \frac{1}{2},-\frac{1}{2},-\frac{1}{2}\right) \\
& t_{4} \Rightarrow(\bar{s} ; \bar{u})=\left(-\frac{1}{2}, \frac{1}{2},-\frac{1}{2} ;-\frac{1}{2},-\frac{1}{2},-\frac{1}{2}\right) .
\end{aligned}
$$

For $-\bar{t}_{\alpha}$ we have:

$$
\begin{aligned}
& -t_{1} \Rightarrow(\bar{s} ; \bar{u})=\left(\frac{1}{2}, \frac{1}{2}, \frac{1}{2} ; \frac{1}{2},-\frac{1}{2}, \frac{1}{2}\right) \\
& -t_{2} \Rightarrow(\bar{s} ; \bar{u})=\left(\frac{1}{2},-\frac{1}{2},-\frac{1}{2} ;-\frac{1}{2},-\frac{1}{2}, \frac{1}{2}\right) \\
& -t_{3} \Rightarrow(\bar{s} ; \bar{u})=\left(-\frac{1}{2},-\frac{1}{2}, \frac{1}{2} ;-\frac{1}{2}, \frac{1}{2}, \frac{1}{2}\right) \\
& -t_{4} \Rightarrow(\bar{s} ; \bar{u})=\left(-\frac{1}{2}, \frac{1}{2},-\frac{1}{2} ; \frac{1}{2}, \frac{1}{2}, \frac{1}{2}\right) .
\end{aligned}
$$

Now by defining the vectors:

$$
\begin{aligned}
& \bar{a} \equiv\left(\frac{1}{2}, \frac{1}{2}, \frac{1}{2}\right) ; \bar{b} \equiv\left(-\frac{1}{2}, \frac{1}{2},-\frac{1}{2}\right) \\
& \bar{c} \equiv\left(\frac{1}{2},-\frac{1}{2},-\frac{1}{2}\right) ; \bar{d} \equiv\left(\frac{1}{2}, \frac{1}{2},-\frac{1}{2}\right) .
\end{aligned}
$$

We may write

$$
\begin{array}{ll}
t_{1} \Rightarrow(\bar{s} ; \bar{u})=(\bar{a} ; \bar{b}) & -t_{1} \Rightarrow(\bar{s} ; \bar{u})=(\bar{a} ;-\bar{b}) \\
t_{2} \Rightarrow(\bar{s} ; \bar{u})=(\bar{c} ; \bar{d}) & -t_{2} \Rightarrow(\bar{s} ; \bar{u})=(\bar{c} ;-\bar{d}) \\
t_{3} \Rightarrow(\bar{s} ; \bar{u})=(-\bar{d} ; \bar{c}) & -t_{3} \Rightarrow(\bar{s} ; \bar{u})=(-\bar{d} ; \bar{c}) \\
t_{4} \Rightarrow(\bar{s} ; \bar{u})=(\bar{b} ;-\bar{a}) & -t_{4} \Rightarrow(\bar{s} ; \bar{u})=(\bar{b} ; \bar{a})
\end{array}
$$

123 
The octet is then made up of the combinations:

$$
(\bar{a} ; \pm \bar{b}) ;(\bar{c} ; \pm \bar{d}) ;(\bar{b} ; \pm \bar{a}) ;(-\bar{d} ; \pm \bar{c}) .
$$

The appearance of octets for basic quantum numbers is reminiscent of elementary particle theory. Thus the Dynamic Theory seems to give promise to the hope of tying elementary particle to fundamental principles in a new way.

D. Mass Conservation Hydrodynamic Systems

The equation of motion for the fifth dimension, mass density, appears as a generalization of the principle of the conservation of mass. Further in classical hydrodynamic systems five equations in five unknowns are used. It seems logical then to expect the five equations of motion appearing in the fivedimensional dynamic theory to be generalizations of the classical equations. An added incentive to investigate the possibilities of this generalization is gained when electromagnetically contained ionized plasmas with mass conversion are considered. For if the five equations are generalizations of the classical hydrodynamic equations, then the use of the five-dimensional fields allowing mass conversion should provide an entirely new viewpoint of a controlled fusion reactor.

Since it is suspected that the five equations of motion resulting from the application of the principle of increasing entropy to a thermo-mechanical system are generalizations of the classical equations, it then becomes necessary to show that this is indeed the case. This seems possible by restricting the system so that it corresponds to the usual system considered.

First, from the dynamic approach, the manifold required for a description of the system is the five-dimensional manifold of space, time, and mass density. Within this manifold the continuity equation no longer holds for the general system. We can, however, restrict our system by first requiring that the system remain on a hypersurface within the five-dimensional manifold. For a system so restricted, any of the five dimensions may be considered as functions of the other four. In particular, since by custom in hydrodynamics the mass density is considered to be a function of space and time, we may consider the mass density to be the variable chosen to be function of the others or

$$
\gamma=\gamma\left(x^{0}, x^{1}, x^{2}, x^{3}\right)
$$


so that

$$
d \gamma=\left(\frac{\partial \gamma}{\partial x^{\alpha}}\right) d x^{\alpha}
$$

Such a system will be constrained to be on a hypersurface embedded within the five-dimensional manifold of space, time, and mass density as shown and upon this hypersurface will be described in a four-dimensional manifold of space and time.

\section{Surface Geometry}

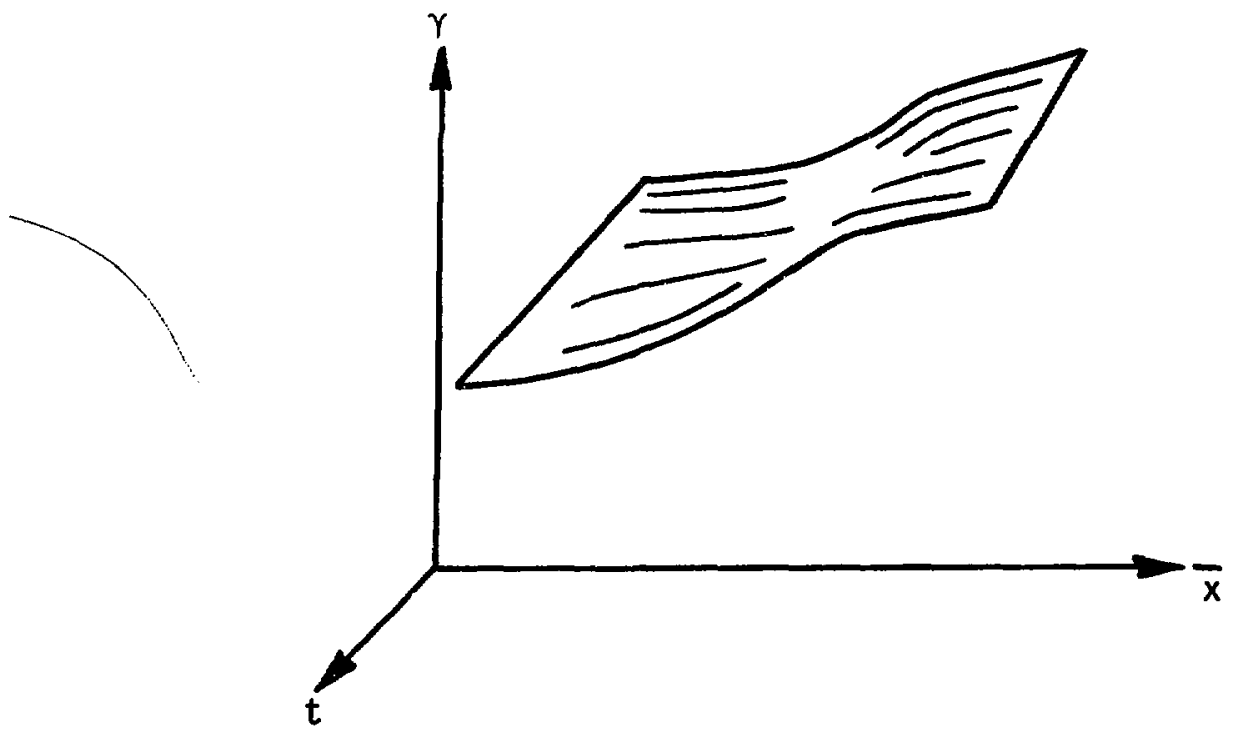

If we further restrict our system by requiring that the total derivative of the mass density to be zero or

$$
d \gamma=0=\frac{d y}{d x^{\alpha}} d x^{\alpha}
$$

then

$$
\frac{d \gamma}{d t}=0=\frac{\partial \gamma}{\partial t}+\frac{\partial \gamma}{\partial x^{1}} v^{1}+\frac{\partial \gamma}{\partial x^{2}} v^{2}+\frac{\partial \gamma}{d x^{3}} v^{3}
$$


or

$$
\frac{\partial \gamma}{\partial t}+\operatorname{grad} \gamma \cdot \vec{v}=0,
$$

which is the usual continuity equation. Thus by restricting the system to this particular hypersurface we have constrained the system to obey the continuity equation as does a usual hydrodynamic system.

Not only does this restriction place our system within the space-time manifold where we may compare the resulting four equations of motion with the equations of motion in relativistic treories but, since the seven gauge field equations must hold in the five-dimensional manifold they must also hold on the hypersurface. This allows the new field quantities to be expressed as functions of the $\bar{E}, \bar{B}$ fields and the partial derivatives of the mass densities. Further, it appears that the additional $B$ field equations may be used to determine $a$ dependence of the $\bar{E}$ and $\bar{B}$ fields upon the mass density and/or its changes.

Then by comparing the equations of motion obtained here for the system restricted to the mass conservation hypersurface with the relativistic NavierStokes equations it should be possible to identify the viscous coefficients with the field quantities and perhaps see how the viscosity depends upon these fields as I feel it does.

Since we have restricted the system to a hypersurface where the mass density is a function of space and time, then the surface is defined by five equations of the type

$$
x^{i}=x^{i}\left(u^{0}, u^{1}, u^{2}, u^{3}\right)
$$

Further, since $x^{4}=y / a_{0}$ and $x^{4}=x^{4}\left(x^{0}, x^{1}, x^{2}, x^{3}\right)$, then Eq. (123) becomes

$$
x^{0}=u^{0}, x^{1}=u^{1}, x^{2}=u^{2}, x^{3}=u^{3}
$$

and

$$
x^{4}=f\left(u^{0}, u^{1}, u^{2}, u^{3}\right)
$$


Since $u^{0}, u^{1}, u^{2}$, and $u^{3}$ are independent variables, the locus defined by Eq. (123) is four-dimensional, and these equations give the coordinates $x^{i}$ of a point on the hypersurface when $u^{0}, u^{1}, u^{2}$ and $u^{3}$ are assigned particular values. This point of view leads one to consider the surface as a four-dimensional manifold $S$ embedded in a five-dimensional enveloping space. We can also study surfaces without reference to the surrounding space, and consider parameters $u^{0}, u^{1}$, $u^{2}$, and $u^{3}$ as coordinates of points in the surface.

If we assign to $u^{0}$ in (123) some fixed value $u^{0}=u_{0}^{0}$, we obtain a threedimensional manifold

$$
x^{i}=x^{i}\left(u_{0}^{0}, u^{1}, u^{2}, u^{3}\right),(i=0,1,2,3,4)
$$

which is a three-dimensional manifold lying on the hypersurface $S$ defined by Eq. (123). By assigning fixed values for any three of the four hypersurface variables we obtain a net of curves, on the hypersurface, which may be called coordinate curves.

Obviously the parametric representation of a hypersurface in the form of (123) is not unique, and there are infinitely many curvilinear coordinate systems which can be used to locate points on a given hypersurface $S$. Thus, if one introduces a transformation

$$
\begin{aligned}
& u^{0}=u^{0}\left(\bar{u}^{0}, \bar{u}^{-1}, \bar{u}^{2}, \bar{u}^{-3}\right), \\
& u^{1}=u^{1}\left(\bar{u}^{0}, \bar{u}^{-1}, \bar{u}^{-2}, \bar{u}^{3}\right), \\
& u^{2}=u^{2}\left(\bar{u}^{0}, \bar{u}^{-1}, \bar{u}^{-2}, \bar{u}^{-3}\right),
\end{aligned}
$$

and

$$
u^{3}=u^{3}\left(\bar{u}^{-0}, \bar{u}^{-1}, \bar{u}^{2}, \bar{u}^{3}\right)
$$

where the $u^{\alpha}\left(\vec{u}^{0}, \bar{u}^{1}, \bar{u}^{2}, \bar{u}^{3}\right)$ are of class $c^{1}$ and are such that the Jacobian 


$$
J=\frac{\partial\left(u^{0}, u^{1}, u^{2}, u^{3}\right)}{\partial\left(\bar{u}^{0}, \bar{u}^{-1}, \bar{u}^{-2}, \bar{u}^{3}\right)}
$$

does not vanish in some region of the variables $\vec{u}^{\alpha}$, then one can insert the values from (125) in (123) and obtain a different set of parametric equations

$$
x^{i}=f^{i}\left(\bar{u}^{0}, \bar{u}^{1}, \bar{u}^{2}, \bar{u}^{3}\right)
$$

defining the hypersurface S. Equation (125) can be looked upon as representing a transformation of coordinates in the hypersurface.

\section{a. First Fundamental Quadratic Form}

The properties of hypersurfaces that can be described without reference to the space in which the hypersurface is embedded are termed "intrinsic" properties. A study of intrinsic properties is made to depend on a certain quadratic differential form describing the metric character of the hypersurface. We proceed to derive this quadratic form for our restricted system.

It will be convenient to adopt certain conventions concerning the meaning of the indices to be used. We will be dealing with two distinct sets of variables: those referring to the five-dimensional space in which the hypersurface is embedded (these are five in number) and with four coordinates $u^{0}, u^{1}, u^{2}$, and $u^{3}$ referring to the four-dimensional manifold $S$. In order not to confuse these sets of variables we shall use Latin letters for the indices referring to the space variables and Greek letters for the hypersurface variables. Thus Latin indices will assume values $0,1,2,3,4$ and Greek indices will have the range of values $0,1,2,3$. A transformation $T$ of space coordinates from one system $\underline{X}$ to another $\underline{\bar{X}}$ will be written as

$$
T=x^{i}=x^{i}\left(\bar{x}^{0}, \bar{x}^{-1}, \bar{x}^{-2}, \bar{x}^{-3}, \bar{x}^{-4}\right) ;
$$

a transformation of Gaussian hypersurface coordinates, such as described by Eq. (125) will be denoted by

$$
u^{\alpha}=u^{\alpha}\left(\bar{u}^{0}, \bar{u}^{1}, \bar{u}^{2}, \bar{u}^{3}\right) .
$$


A repeated Greek index in any term denotes the sumration from 0 to 3 ; a repeated Latin index represents the sum from 0 to 4 . Unless a statement to the contrary is made, we shall suppose that all functions appearing in the discussion are of class $c^{2}$ in the regions of their definitions.

Consider the hypersurface $S$ defined by

$$
x^{i}=x^{i}\left(u^{0}, u^{1}, u^{2}, u^{3}\right),
$$

where the $x^{i}$ are coordinates covering the five-dimensional space in which the hypersurface $S$ is embedded, and a curve $C$ on $S$ defined by

$$
u^{\alpha}=u^{\alpha}(\tau), \tau_{1} \leq \tau \leq \tau_{2}
$$

where the $u^{\alpha_{1}} s$ are the Gaussian coordinates covering $S$. Viewed from the surrounding space, the curve defined by $(128)$ is a curve in a five-dimensional manifold, which we shall assume, for the present, is Riemannian entropy manifold of the Dynamic Theory, and its element of arc is given by the formula

$$
\left\langle d q^{0}\right\rangle^{2}=\hat{g}_{i j} d x^{i} d x^{j}
$$

From (127) we have

$$
d x^{i}=\frac{\partial x^{i}}{\partial u^{\alpha}} d u^{\alpha}
$$

where, as is clear from (128),

$$
d u^{\alpha}=\frac{d u^{\alpha}}{d \tau} d \tau
$$

Substituting from (129) and (130), we get

$$
\left(d q^{0}\right)^{2}=\hat{g}_{i j} \frac{\partial x^{i}}{\partial u^{\alpha}} \frac{\partial x^{j}}{\partial u^{\beta}} d u^{\alpha} d u^{\beta}
$$




$$
=A_{\alpha \beta} d u^{\alpha} d u^{\beta} \text {, }
$$

where

$$
A_{\alpha \beta} \equiv \hat{g}_{i j} \frac{\partial x^{i}}{\partial u^{\alpha}} \frac{\partial x^{j}}{\partial u^{\beta}}
$$

The expression for $\left(\mathrm{dq}^{0}\right)^{2}$, namely

$$
\left(d q^{0}\right)^{2}=A_{\alpha \beta} d u^{\alpha} d u^{\beta},
$$

is the square of the linear element of C lying on the hypersurface $S$, and the right hand member of (131) can be called the First Fundamental quadratic form of the hypersurface. The length of arc of the curve is given by

$$
q_{2}^{0}-q_{1}^{0}=\int_{\tau_{1}}^{\tau_{2}} \gamma \sqrt{A} \overline{\alpha \beta} \overline{\dot{u}^{\alpha} \dot{b}^{\beta}} d \tau,
$$

where $\dot{u}^{\alpha}=\frac{d u^{\alpha}}{d \tau}$ and $q^{0}$ is the specific entropy. The total change in the entropy along the curve $C$ would then be

$$
\gamma\left(q_{2}^{0}-q_{1}^{0}\right)=\int_{\tau_{1}}^{\tau_{2}} \gamma \sqrt{A_{\alpha \beta} u^{\alpha} u^{\beta}} d \tau
$$

Consider a transformation of surface coordinates

$$
u^{\alpha}=u^{\alpha}\left(\bar{u}^{0}, \bar{u}^{1}, \bar{u}^{2}, \bar{u}^{3}\right)
$$

with a non-vanishing Jacobian

$$
J=\left|\frac{\partial u^{\alpha}}{\partial u^{\beta}}\right| \text {. }
$$


It follows from (134) that

$$
d u^{\alpha}=\frac{\partial u^{\alpha}}{\partial \bar{u}^{\beta}} d \bar{u}^{\beta},
$$

and hence (132) yields

$$
\left(d q^{0}\right)^{2}=A_{\alpha \beta} \frac{\partial u^{\alpha}}{\partial \bar{u}^{\gamma}} \frac{\partial u^{\beta}}{\partial \bar{u}^{\delta}} d \bar{u}^{\gamma} d \bar{u}^{\delta}
$$

If we set

$$
A_{\gamma \delta}=A_{\alpha \beta} \frac{\partial u^{\alpha}}{\partial \bar{u}^{\gamma}} \frac{\partial u^{\beta}}{\partial \bar{u}^{-\delta}},
$$

we see that the set of quantities $A_{\alpha \beta}$ represents a symmetric covariant tensor of rank two with respect to the admissible transformations (134) of hypersurface coordinates. The fact that the $A_{\alpha \beta}$ are components of a tensor is also evident from (132), since $\left(d q^{0}\right)^{2}$ is an invariant and the quantities $A_{\alpha \beta}$ are symetric. The tensor $A_{\alpha \beta}$ is called the covariant metric tensor of the hypersurface.

Since the form (132) is positive definite, the determinant

$$
A=\left|A_{\alpha \beta}\right|>0
$$

and we can define the reciprocal tensor $A^{\alpha \beta}$ by the formula $A^{\alpha \beta} A_{B \gamma}=\delta_{\gamma}^{\alpha}$. The properties of surfaces concerning the study of the first fundamental quadratic form

$$
\left(d q^{0}\right)^{2}=A_{\alpha \beta} d u^{\alpha} d u^{\beta}
$$

constitute a body of what is known as the "intrinsic geometry of surfaces." They take no account of the distinguishing characteristics of surfaces as they might appear to an observer located in the surrounding space. Two surfaces, a cylinder 
and a cone, for example, appear to be entirely different when viewed from the enveloping space, and yet their intrinsic geometries are completely indistinguishable since the metric properties of cylinders and cones can be described by the identical expressions for square of the element of arc. If a coordinate system exists on each of the two surfaces such that the linear elements on them are characterized by the same metric coefficients $A_{\alpha \beta}$, the surfaces are called "isometric."

Thus if our description of the restricted system is done only in terms of the intrinsic geometry of the hypersurface we may lose sight of features which may characterize our system when viewed from the enveloping space. Therefore, in order to characterize the shape of the surface we must develop a yiew which involves the enveloping space.

\section{b. Second Fundamental Quadratic Form}

An entity that provides a characteristic of the shape of the surface as it appears from the enveloping space is the normal 1 ine to the surface. The behavior of the normal line as its foot is displaced along the surface depends on the shape of the surface, and it occurred to Gauss to describe certain properties of surfaces with the aid of a quadratic form that depends in a fundamental way on the behavior of the normal line. Before we introduce this new quadratic form let us recall the definition (131),

$$
A_{\alpha \beta} \equiv \hat{g}_{\mathbf{i j}} \frac{\partial x^{i}}{\partial u^{\alpha}} \frac{\partial x^{j}}{\partial u^{\beta}}(\mathbf{i}, \mathbf{j}=0,1,2,3,4)(\alpha, \beta=0,1,2,3) .
$$

We note that the foregoing formulas depend on both the Latin and Greek indices, and we recall that the Latin indices run from 0 to 4 and refer to the surrounding space, whereas the Greek indices assume values $0,1,2$, and 3 and are associated with the embedded hypersurface. Furthermore, the $\mathbf{d x}{ }^{i}$ and $\hat{\mathbf{g}}_{\mathbf{i j}}{ }^{\prime} \mathbf{s}$ are tensors with respect to the transformations induced on the space variables $x^{j}$, whereas such quantities $d u^{\alpha}$ and $A_{\alpha \beta}$ are tensors with respect to the transformation of Gaussian surface coordinates $u^{\alpha}$. Equation (131) is a curious one since it contains partial derivatives $\frac{\partial x^{i}}{\partial u^{\alpha}}$ depending on both Latin and Greek indices. Since both $A_{\alpha \beta}$ and $\hat{g}_{i j}$ in (131) are tensors, this formula suggests 
that $\frac{\partial x^{i}}{\partial u^{\alpha}}$ can be regarded either as a contravariant space vector or as a covariant surface vector. Let us investigate this set of quantities more closely.

Let us take a small displacement on the hypersurface $S$, specified by the surface vector $d u^{\alpha}$. The same displacement, as is clear from (130), is described by the space vector with components

$$
d x^{i}=\frac{\partial x^{i}}{\partial u^{\alpha}} d u^{\alpha}
$$

The left-hand member of this expression is independent of the Greek indices, and hence it is invariant relative to a change of the surface coordinates $u^{\alpha}$. Since $d u^{\alpha}$ is an arbitrary surface vector, we conclude that

$$
\frac{\partial x^{i}}{\partial u^{\alpha}}
$$

is a covariant surface vector. On the other hand, if we change the space coordi.. nates, the $\mathrm{du}^{\alpha}$, being a surface vector, is invariant relative to this change, so the (136) must be a contravariant space vector. Hence we can write (136) as

$$
x_{\alpha}^{i} \equiv \frac{\partial x^{i}}{\partial u^{\alpha}}
$$

where the indices properly describe the tensor character of this set of quantities.

Let $\bar{A}$ and $\bar{B}$ be a pair of surface vectors drawn from one point $P$ of $S$.

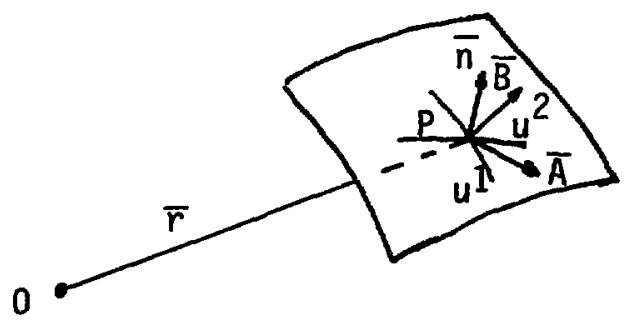


Then using (137) they can be represented in the form

$$
A^{i}=x_{\alpha}^{i} A^{\alpha} \text { and } B^{i}=x_{\alpha}^{i} B^{\alpha}
$$

The five-dimensional vector product, defined by

$$
N^{k} \equiv+\varepsilon^{k i j} A_{i} B_{j}
$$

is the vector normal to the tangent plane determined by the vectors $\bar{A}$ and $\bar{B}$, and the unit vector $\bar{n}$ perpendicular to the tangent plane, so oriented that $\bar{A}$, $\bar{B}$, and $\bar{n}$ form a right-handed system, is

$$
\bar{n}=\frac{\varepsilon^{k i j} A_{i} B_{j}}{\left|\varepsilon^{\alpha \beta} A_{\alpha} B_{B}\right|}
$$

We call the vector $\bar{n}$ the unit normal vector to the hypersurface $S$ at $P$. Clearly, $\bar{n}$ is a function of coordinates $\left(u^{0}, u^{1}, u^{2}, u^{3}\right)$, and as the point $P$ $\left(u^{0}, u^{1}, u^{2}, u^{3}\right)$ is displaced to a new position $P\left(u^{0}+d u^{0}, u^{1}+d u^{1}, u^{2}+d u^{2}\right.$, $\left.u^{3}+d u^{3}\right)$, the vector $\bar{n}$ undergoes a change

$$
d \bar{n}=\frac{\partial \bar{n}}{\partial u^{\alpha}} d u^{\alpha}
$$

whereas the position vector $\bar{r}$ is changed by the amount

$$
d \bar{r}=\frac{\partial \bar{r}}{\partial u^{\alpha}} d u^{\alpha} .
$$

Let us form the scalar product

$$
d \bar{n} \cdot d \bar{r}=\frac{\partial \bar{n}}{\partial u^{\alpha}} \cdot \frac{\partial \bar{r}}{\partial u^{\beta}} d u^{\alpha} d u^{\beta} .
$$


If we define

$$
b_{\alpha \beta}=\frac{1}{2}\left(\frac{\partial \bar{n}}{\partial u^{\alpha}} \cdot \frac{\partial \bar{r}}{\partial u^{\beta}}+\frac{\partial \bar{n}}{\partial u^{\beta}} \cdot \frac{\partial \bar{r}}{\partial u^{\alpha}}\right)
$$

so that (142) reads

$$
d \bar{n} \cdot d \bar{r}=-b_{\alpha \beta} d u^{\alpha} d u^{\beta}
$$

the left-hand member of (143), being the scalar product of two vectors in a Riemannian space by being in the entropy manifold, is an invariant; moreover, from symmetry with respect to $\alpha$ and $\beta$, it is clear that the coefficients $d u^{\alpha} d u^{\beta}$ in the right-hand member of (143) define a covariant tensor of rank two. The quadratic form

$$
B \equiv \mathrm{b}_{\alpha \beta} \mathrm{du} \mathrm{u}^{\alpha} \mathrm{du} \mathrm{u}^{\beta}
$$

called the second fundamental quadratic form of the hypersurface, will be shown to play an essential part in the study of hypersurfaces when they are viewed from the surrounding space, just as the first fundamental quadratic form $A \equiv$ $d \bar{r} \cdot d \bar{r}$, or

$$
A=A_{\alpha \beta} d u^{\alpha} d u^{\beta},
$$

did in the study of intrinsic properties of a hypersurface.

We can rewrite the formula (140) in terms of the components $x_{\alpha}^{i}$ of the base vectors $\bar{a}_{\alpha}$. We denote the covariant components of $\bar{n}$ by $n^{i}$ and observe that its covariant components $n_{i}$ are given by

$$
n_{i}=\frac{\varepsilon_{i j k} A^{j_{B} k}}{\bar{A} \bar{B} \sin \theta}
$$


and

$$
A B \sin \theta=\varepsilon_{\alpha \beta} A^{\alpha} A^{\beta} \text {. }
$$

Substituting in (145) from (138) and (146), we get

$$
\left(n_{j} \varepsilon_{\alpha \beta}-\varepsilon_{i j k} x_{\alpha}^{j} x_{\beta}^{k}\right) A^{\alpha} B^{\beta}=0
$$

and, since this relation is valid for all surface vectors, we conclude that

$$
n_{i} \varepsilon_{\alpha \beta}=\varepsilon_{i j k} x_{\alpha}^{j} x_{\beta}^{k}
$$

Multiplying (147) through $\varepsilon^{\alpha \beta}$, and noting that $\varepsilon^{\alpha \beta} \varepsilon_{\alpha \beta}=2$, we get the desired result

$$
n_{i}=\frac{1}{2} \varepsilon^{\alpha \beta} \varepsilon_{i j k} x_{\alpha}^{j} x_{\beta}^{k}
$$

It is clear from the structure of this formula that $n_{i}$ is a space vector which does not depend on the choice of surface coordinates. This fact is also obvious from purely geometric considerations.

$$
\text { c. Tensor Derivatives }
$$

We wish to reduce the second fundamental quadratic form (144) analytically by the operation of tensor differentiation of tensor fields which are functions of both surface and space coordinates. To do this we shall first present the concept of tensor differentiation introduced by A. J. McConne11*.

Let us consider a curve $C$ lying on a given hypersurface $S$ and a vector $A^{j}$ defined along $C$. If $\tau$ is a parameter along $C$, we can compute the intrinsic derivative $\frac{\delta A^{i}}{\delta \tau}$ of $A^{i}$, namely,

*A. J. McConne11, Absolute Differential Calculus, London, 1931, Chapters XIV-XVI. 


$$
\left.\frac{\delta A^{i}}{\delta \tau}=\frac{d A^{i}}{d t}+\hat{o}_{j}^{\{}:_{j}^{i}\right\} A^{j} \frac{d x^{k}}{d \tau}
$$

In formula (149) the Christoffel symbols $\hat{g}_{j k}^{[j}$ refer to the space coordinates $x^{i}$ and are formed from the metric coefficients $\hat{g}_{i j}$. This is indicated by the prefix $\hat{g}$ on the symbol. On the other hand, if we consider a surface vector $A^{\alpha}$ defined along the same curve $C$, we can form the intrinsic derivative with respect to the surface variables, namely,

$$
\left.\frac{\delta A^{\alpha}}{\delta \tau}=\frac{d A^{\alpha}}{d \tau}+{ }_{a}{ }_{B \gamma}^{\alpha}\right\} A^{\beta} \frac{d u^{\gamma}}{d \tau}
$$

In this expression the Christoffel symbols ${ }_{\alpha}\left\{\begin{array}{c}\alpha \\ \beta\end{array}\right\}$ are formed from the metric coefficients $a_{\alpha \beta}$ associated with the Gaussian hypersurface coordinates $u^{\alpha}$. A geometric interpretation of these formulas is at hand when the fields $A^{j}$ and $A^{\alpha}$ are such that $\frac{\delta A^{i}}{\delta \tau}=0$ and $\frac{\delta A^{\alpha}}{\delta \tau}=0$. In the first equation the vectors $A^{i}$ form a parallel field with respect to $C$, considered as a space curve, whereas the equation $\frac{\delta A^{\alpha}}{\delta \tau}=0$ defines a parallel field with respect to $C$ regarded as a surface curve. The corresponding formulas for the intrinsic derivatives of the covariant vectors $A_{i}$ and $A_{\alpha}$ are

$$
\left.\frac{\delta A_{i}}{\delta \tau}=\frac{d A_{i}}{d \tau}-\hat{g}^{\{}{ }_{i j}^{k}\right\} A_{k} \frac{d x^{j}}{d \tau}
$$

and

$$
\left.\frac{\delta A_{\alpha}}{\delta \tau}=\frac{d A_{\alpha}}{d \tau}-a_{\alpha \beta}^{\{}\right\} A_{\gamma} \frac{d u^{B}}{d \tau}
$$

Consider next a tensor field $T_{\alpha}^{i}$, which is a contravariant vector with respect to a transformation of space coordinate $x^{i}$ and a covariant vector relative to a transformation of surface coordinates $u^{\alpha}$. An example of a field of this type is the tensor $x_{\alpha}^{i}=\frac{\partial x^{i}}{\partial n^{\alpha}}$ introduced earlier. If $T_{\alpha}^{i}$ is defined over a surface 
curve $C$, and the parameter along $C$ is $\tau$, then $T_{\alpha}^{i}$ is a function of $\tau$. We introduce a parallel vector field $A_{j}$ along $C$, regarded as a space curve, and a parallel vector field $B^{\alpha}$ along $C$, viewed as a surface curve, and form an invariant

$$
\Phi(\tau)=T_{\alpha}^{i} A_{i} B^{\alpha}
$$

The derivative of $\Phi(\tau)$ with respect to the parameter $\tau$ is given by the expression

$$
\frac{d \Phi}{d \tau}=\frac{d T_{\alpha}^{i}}{d \tau} A_{i} B^{\alpha}+T_{\alpha}^{i} \frac{d A_{i}}{d \tau} B^{\alpha}+T_{\alpha}^{i} A_{i} \frac{d B^{\alpha}}{d \tau}
$$

which is obviously an invariant relative to both the space and surface coordinates. But, since the fields $A_{j}(\tau)$ and $B^{\alpha}(\tau)$ are parallel,

$$
\left.\frac{d A_{i}}{d \tau}=\hat{g}^{l} \underset{j j}{k}\right\} A_{k} \frac{d x^{j}}{d \tau} \text { and } \frac{d B^{\alpha}}{d \tau}=-a^{-\{} \begin{gathered}
\alpha \\
B \gamma
\end{gathered} B^{\beta} \frac{d u^{\gamma}}{d \tau} \text {, }
$$

and (153) becomes

$$
\left.\frac{d \Phi}{d \tau}=\left[\frac{d T_{\alpha}^{i}}{d \tau}+\hat{g}_{j k}^{i}{ }_{j k}^{i} T_{\alpha}^{j} \frac{d x^{k}}{d \tau}-\bar{a}_{\alpha \gamma}^{-\delta}\right\} T_{\delta}^{i} \frac{d u^{\gamma}}{d \tau}\right] A_{i} B^{\alpha}
$$

Since this is invariant for an aribitrary choice of parallel fields $A_{j}$ and $B^{\alpha}$, the quotient law guarantees that the expression in the brackets of (150) is a tensor of the same character as $T_{\alpha}^{i}$. We call this tensor the intrinsic tersor derivative of $T_{\alpha}^{i}$ with respect to the parameter $\tau$, and write

$$
\left.\frac{\delta T_{\alpha}^{i}}{\delta t} \equiv \frac{d T_{\alpha}^{i}}{d \tau}+{ }_{\hat{g}^{[}}^{\left.f_{j k}^{j}\right\}} T_{\alpha}^{j} \frac{d x^{k}}{d \tau}={ }_{a}^{\{}{ }_{\alpha \gamma}^{\delta}\right\} T_{\delta}^{i} \frac{d u^{\gamma}}{d \tau} .
$$

If the field $T_{\alpha}^{i}$ is defined over the entire hypersurface $S$, we can argue that, since 


$$
\left.\left.\frac{\delta T_{\alpha}^{i}}{\delta \tau} \equiv\left[\frac{\partial T_{\alpha}^{i}}{\partial u^{\gamma}}+g^{\{}{ }_{j k}^{i}\right\} T_{\alpha}^{j} j_{\gamma}^{k}-a_{\gamma \alpha}{ }_{\gamma \alpha}^{\delta}\right\} T_{\delta}^{i}\right] \frac{d u^{\gamma}}{d \tau}
$$

is a tensor field and $\frac{d u^{\gamma}}{d \tau}$ is an arbitrary surface vector (for $C$ is arbitrary), the expression in the bracket is a tensor of the type $T_{\alpha \gamma}^{i}$. We write

$$
\left.\left.T_{\alpha \gamma}^{i} \equiv \frac{\partial T_{\alpha}^{i}}{\partial u^{\gamma}}+\hat{g}^{\{j k}{ }_{j k}^{i}\right\} T_{\alpha}^{j} x_{\gamma}^{k}-a_{\alpha \gamma}^{\tau_{\alpha}^{\delta}}\right\} T_{\delta}^{i}
$$

and call $T_{\alpha, \gamma}^{i}$ the tensor derivative of $T_{\alpha}^{i}$ with respect to $u^{\gamma}$.

The extension of this definition to more complicated tensors is obvious from the structure of (156). Thus the tensor derivative of $T_{\alpha \beta}^{i}$ with respect to $u^{\gamma}$ is given by

$$
\left.\left.\left.T_{\alpha \beta, \gamma}^{j}=\frac{\partial T_{\alpha \beta}^{j}}{\partial u^{\gamma}}+g^{\{j k} j^{j}\right\} T_{\alpha \beta}^{j} x_{\gamma}^{k}-a^{\{} \frac{\delta}{\delta}\right\} T_{\delta \beta}^{j}-a^{\{}{ }_{\beta \gamma}^{\delta}\right\} T_{\alpha \delta}^{j} .
$$

If the surface coordinates at any point $\mathrm{P}$ or $\mathrm{S}$ are geodesic, and the space coordinates are orthogonal Cartesian, we see that at that point the tensor derivatives reduce to the ordinary derivatives. This leads us to conclude that the operations of tensor differentiations of products and sums follow the usual rules and that the tensor derivatives of $\hat{g}_{i j}, A_{\alpha \beta}, \varepsilon_{i j k}, \varepsilon_{\alpha \beta}$ and their associated tensors vanish. Accordingly, they behave as constants in the tensor differentiation.

The apparatus developed in the preceding section permits us to obtain easily and in the most general form an important set of formulas due to Gauss. We will also deduce with its aid the second fundamental quadratic form of a surface a) ready encountered.

We begin by calculating the tensor derivative of the tensor $x_{\alpha}^{\mathbf{i}}$, representing the components of the surface base vectors $\bar{a}_{\alpha}$. We have 


$$
\left.x_{\alpha \beta}^{j}=\frac{\partial^{2} x^{j}}{\partial u^{\alpha} \partial u^{\beta}}+\hat{g}^{\{j k}{ }_{j k}^{i} x_{\alpha}^{j} x_{\beta}^{k}-a_{\alpha \beta}^{\{}\right\} x_{\delta}^{j},
$$

from which we deduce that

$$
x_{\alpha, \beta}^{\mathfrak{j}}=x_{\beta, \alpha}^{\mathfrak{j}}
$$

Since the tensor derivative of $a_{\alpha \beta}$ vanishes, we obtain, upon differentiating the relation

$$
\begin{aligned}
& A_{\alpha \beta}=\hat{g}_{i j} x_{\alpha}^{j} x_{\beta}^{j}, \\
& \hat{g}_{i j} x_{\alpha, \gamma}^{i} x_{\beta}^{j}+g_{i j} x_{\alpha}^{i} x_{\beta, \gamma}^{j}=0 .
\end{aligned}
$$

Interchanging $\alpha, \beta, \gamma$ cyclically leads to two formulas:

$$
\hat{g}_{i j} x_{\beta, \alpha}^{j} x_{\gamma}^{j}+\hat{g}_{i j} x_{\beta}^{i} x_{\gamma, \alpha}^{j}=0
$$

and

$$
\hat{g}_{i j} x_{\gamma, \beta}^{i} x_{\alpha}^{j}+\hat{g}_{i j} x_{\gamma}^{i} x_{\alpha, \beta}^{j}=0
$$

If we add (160) and (161), subtract (159), and take into account the symmetry relation (158), we obtain

$$
g_{i j} x_{\alpha, \beta}^{i} x_{\gamma}^{j}=0
$$

This is the orthogonality relation which states that $x_{\alpha, \beta}^{j}$ is a space vector normal to the surface, and hence it is directed along the unit norma $n^{i}$. Consequentiy, there exists a set of functions $b_{\alpha \beta}$ such that 


$$
x_{\alpha, \beta}^{i}=b_{\alpha \beta} n^{i}
$$

The quantities $b_{\alpha \beta}$ are the components of a symmetric surface tensor, and the differential quadratic form

$$
B \equiv \mathbf{b}_{\alpha \beta} \mathbf{d} u^{\alpha} \mathrm{du} \mathbf{u}^{\beta}
$$

is the desired second fundamental form.

$$
\begin{aligned}
& \text { Now since } n_{j} n^{i}=\delta_{j}^{i} \text {, and } n_{i}=\hat{g}_{i j} n^{j} \text {, then } \\
& b_{\alpha \beta}=\hat{g}_{i j} x_{\alpha, \beta}^{i} n^{j} ;
\end{aligned}
$$

but since $n_{i}=\frac{1}{2} \varepsilon^{\alpha \beta} \varepsilon_{i j k} x_{\alpha}^{j} x_{\beta}^{k}$, then

$$
\mathbf{b}_{\alpha \beta}=\frac{1}{2} \varepsilon^{\gamma \delta} \varepsilon_{i j k} x_{\alpha, \beta}^{i} x_{\gamma}^{j} x_{\delta}^{k} .
$$

We now have, in Eqs. (131) and (162), the formulas necessary to determine the first and second fundamental quadratic forms for our system constrained to a four-dimensional hypersurface. Our objective is to show that by appropriately constraining our system we arrive at the Navier-Stokes equations. Let us determine the first fundamental quadratic form.

First recall that our system was restricted so that $x^{4}=x^{4}\left(x^{0}, x^{1}, x^{2}, x^{3}\right)$ or the mass density is a function of space and time; then we have the relations

$$
\begin{aligned}
& x^{0}=u^{0}, \\
& x^{1}=u^{1}, \\
& x^{2}=u^{2}, \\
& x^{3}=u^{3},
\end{aligned}
$$

and 


$$
x^{4}=f\left(x^{0}, x^{1}, x^{2}, x^{3}\right)=f\left(u^{0}, u^{1}, u^{2}, u^{3}\right)
$$

Since Eq. (131) is

$$
A_{\alpha \beta}=\hat{g}_{i j} \frac{\partial x^{j}}{\partial u^{\alpha}} \frac{\partial x^{j}}{\partial u^{\beta}}=\hat{g}_{i j} x_{\alpha}^{i} x_{\beta}^{j},
$$

then

$$
A_{00}=\hat{g}_{00}=2 \hat{g}_{04} f_{0}+\hat{g}_{44}\left(f_{0}\right)^{2},
$$

where

$$
f_{0} \equiv \frac{\partial f}{\partial u^{0}}
$$

In a similar fashion we may determine the remaining coefficients so that

$$
a_{\alpha 3}=\left|\begin{array}{llll}
\hat{g}_{00}+2 \hat{g}_{04} f_{0}+\hat{g}_{44}\left(f_{0}\right)^{2} & \hat{g}_{01}+2 \hat{g}_{04} f_{1}+\hat{g}_{44} f_{0} f_{1} & \hat{g}_{02}+2 \hat{g}_{04} f_{2}+\hat{g}_{44} f_{0} f_{2} & \hat{g}_{03}+2 \hat{g}_{04} f_{3}+\hat{g}_{44} f_{0} f_{3} \\
\hat{g}_{04} \hat{g}_{1}+\hat{g}_{44} f_{0} f_{1} & \hat{g}_{11}+2 \hat{g}_{14} f_{1}+\hat{g}_{44}\left(f_{1}\right)^{2} & \hat{g}_{12}+2 \hat{g}_{14} f_{2}+\hat{g}_{44} f_{1} f_{2} & \hat{g}_{13}+2 \hat{g}_{14} f_{3}+\hat{g}_{44} f_{1} f_{3} \\
\hat{g}_{02}+2 \hat{g}_{04} f_{2}+\hat{g}_{44} f_{0} f_{2} & \hat{g}_{12}+2 \hat{g}_{14} f_{2}+\hat{g}_{44} f_{1} f_{2} & \hat{g}_{22}+2 \hat{g}_{24} f_{2}+\hat{g}_{44}\left(f_{2}\right)^{2} & \hat{g}_{23}+2 \hat{g}_{24} f_{3}+\hat{g}_{44} f_{2} f_{3} \\
\hat{g}_{03}+2 \hat{g}_{04} f_{3}+\hat{g}_{44} f_{0} f_{3} & \hat{g}_{13}+2 \hat{g}_{14} f_{3}+\hat{g}_{44} f_{1} f_{3} & \hat{g}_{23}+2 \hat{g}_{24} f_{3}+\hat{g}_{44} f_{2} f_{3} & \hat{g}_{33}+2 \hat{g}_{34} f_{3}+\hat{g}_{44}\left(f_{3}\right)^{2}
\end{array}\right|
$$

Obviously from this determination we may write the metric coefficients of the first fundamental quadratic form as the sum of two tensors, or

$$
A_{\alpha \beta}=\hat{g}_{\alpha \beta}+h_{\alpha \beta} ; \alpha, \beta=0,1,2,3,
$$


where the $h_{\alpha \beta}$ are functions of the partial derivatives of the mass density with respect to space and time in addition to space and time from the $\hat{g}_{j 4}$ where $i=$ $0,1,2,3,4$.

Though we may use Eq. (162) to determine the metric coefficients for the second fundamental quadratic form, it is not necessary for the current presentation.

The hypersurface which is embedded in the five-dimensional space is a fourdimensional curvilinear space-time manifold. Thus the relativistic hydrodynamic equations are applicable here so long as the metric coefficients are determined as coefficients of the hypersurface quadratic form.

The complete energy-momentum tensor for a fluid in a flat Riemannian spacetime manifold is given by*

$$
T^{\alpha \beta}=\gamma \dot{u}^{\alpha} \dot{u}^{\beta}+\frac{p}{c^{2}}\left(\dot{u}^{\alpha \cdot \beta}-g^{\alpha \beta}\right)
$$

where $\dot{u}^{\alpha} \equiv \frac{d u^{\alpha}}{d s}, s$ is the arc length. Then based upon this energy-momentum tensor the flow of a fluid under the effect of its own internal pressure force is given by setting the divergence of (165) equal to zero, or

$$
T^{\alpha \beta}{ }_{\beta}=0
$$

If we reduce Eq. (165) to the non-relativistic limit, the use of (166) gives us

$$
\mathrm{g}^{\beta \delta} \tau_{\alpha \beta, \delta}=\gamma a_{\alpha}, \alpha, \beta, \delta=1,2,3,
$$

where $\tau^{\alpha \beta}=\mathrm{Pg}^{\alpha \beta}$ is the three-dimensional stress tensor of an ideal fluid.

If in Eq. (165) we use the fact that the metric coefficients for the hypersurface may be written as the sum of (164), then we have

$$
T^{\alpha \beta}=\gamma \dot{u}^{\alpha} \dot{u}^{\beta}+\frac{p}{c^{2}}\left(\dot{u}^{\alpha} \dot{u}^{\beta}-\hat{g}^{\alpha \beta}-h^{\alpha \beta}\right),
$$

*R. Adler, M. Bazin, and M. Schiffer, Introduction to General Relativity, 2nd Ed. (McGraw-Hi11, New York, 1975), p. 337. 
where it must be remembered that the $\dot{u}^{\alpha} \dot{u}^{\beta}$ are also dependent upon this same sum. In the nonrelativistic limit the effects of this sum of metric tensors appear as a sum in the stress tensor

$$
\tau^{\alpha \beta}=-P \hat{g}^{\alpha \beta}-P h^{\alpha \beta}, \alpha, \beta=1,2,3 .
$$

Recall that the $\hat{g}^{\alpha \beta}$ refer to the three-dimensional space viewed from the five-dimensional manifold. The $h^{\alpha \beta}$, however, contain the information about the surface embedded in the five-dimensional space. If we then associate the tensor

$$
t^{\alpha \beta} \equiv-P h^{\alpha \beta}
$$

with the yiscous stresses, we are saying that the viscous stresses depend upon the geometric character of the hypersurface.

In the limit of sma11 displacements we write the strain velocity tensor as

$$
\dot{e}_{\alpha \beta}=\frac{1}{2}\left(v_{\alpha, \beta}+v_{\beta, \alpha}\right) \text {. }
$$

Then the first order coefficients of viscosity. are related to the strain velocity tensor and viscous stresses according to

$$
t^{\alpha \beta}=\frac{c^{\alpha \beta \delta n}}{2}\left(v_{\delta, n}+v_{n, \delta}\right)
$$

If we then use (170) in (171), we find that the relationship between the geometric character of the hypersurface and the viscous coefficients is given by

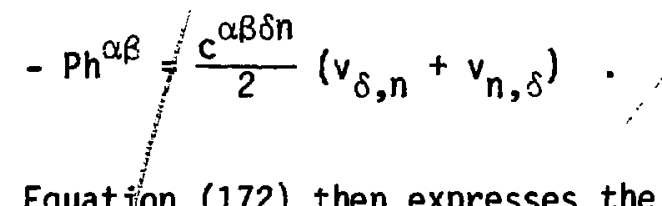

Equation (172) then expresses the functional dependence of the viscous coefficients upon the strain velocities, pressure, mass density, and their derivatives. 
2. Relativistic Hydrodynamics. By viewing classical hydrodynamics to be given by the embedding of a four-dimensional hypersurface within a fivedimensional manifold, the association (172) between the geometric properties of the hypersurface and the viscous coefficients could be tentatively made. We may now go back and develop this relationship more comp?etely.

The hypersurface, which becomes embedded in the five-dimensional manifold by the restriction that $x^{4}=x^{4}\left(x^{0}, x^{1}, x^{2}, x^{3}\right)$ is a four-dimensional relativistic manifold. Thus for the surface we may use the relativistic energy-momentum tensor, which is

$$
T^{\mu \nu}=\gamma u^{\mu \nu}+\frac{p}{c^{2}}\left(u^{\mu} u^{\nu}-g^{\mu \nu}\right),
$$

where $u^{\mu} \equiv \frac{d x^{\mu}}{d x^{0}}$ and $\mu, v=0,1,2,3$. The divergence of (173) yields the flow equations for a fluid under the effects of its own internal pressure.

However, from the viewpoint of the Dynamic Theory, the surface metric coefficients may be written in terms of the metric coefficients of the first four space coordinates as given by Eqs. (164) and (165), or

$$
A_{\alpha \beta}=\hat{g}_{\alpha \beta}+h_{\alpha \beta}, \quad \alpha, \beta=0,1,2,3 \text {. }
$$

Thus the square of the arc length for the entropy manifold may be written as

$$
\left(d q^{0}\right)^{2}=A_{\alpha \beta} d x^{\alpha} d x^{\beta}=\hat{g}_{\alpha \beta} d x^{\alpha} d x^{\beta}+h_{\alpha \beta} d x^{\alpha} d x^{\beta}
$$

or, if $u^{\alpha} \equiv \frac{d x^{\alpha}}{d q^{0}}$, then

$$
1=A_{\alpha \beta} u^{\alpha} u^{\beta}=\hat{g}_{\alpha \beta} u^{\alpha} u^{\beta}+h_{\alpha \beta} u^{\alpha} u^{\beta} \text {. }
$$

Then on the hypersurface the energy momentum tensor would become

$$
T^{\alpha \beta}=\dot{\gamma} u^{\alpha} u^{\beta}+\frac{P}{c^{2}}\left(u^{\alpha} u^{\beta}-A^{\alpha \beta}\right)
$$


or

$$
T^{\alpha \beta}=\gamma u^{\alpha} u^{\beta}+\frac{p}{c^{2}}\left(u^{\alpha} u^{\beta}-\hat{g}_{\alpha \beta}-h^{\alpha \beta}\right)
$$

Since the surface coordinates, $x^{\alpha}$, are the same as the first four coordinates of the surrounding space, the velocities $u^{\alpha}$ are the same whether considered as surface or space vectors. The difference between the surface view and a fourdimensional space view appears in the metric coefficients. Thus while the square of the arc element on the surface is unity, the square of the arc element in the surrounding space is not, or

$$
I=A_{\alpha \beta} u^{\alpha} u^{\beta}
$$

but

$$
\hat{g}_{\alpha \beta} u^{\alpha} u^{\beta}=1-h_{\alpha \beta} u^{\alpha} u^{\beta}
$$

3. Classical Hydrodynamics. Suppose we consider $\hat{g}_{\alpha \beta}$ to be a flat space then because of (174) we may write

$$
T^{\alpha \beta}=\gamma u^{\alpha} u^{\beta}+\frac{p}{c^{2}}\left(u^{\alpha} u^{\beta}-\hat{g}^{\alpha \beta}\right)-\frac{p}{c^{2}} h^{\alpha \beta} \text {. }
$$

If we then form the space divergence

$$
T^{0 \nu}, v=\frac{1}{c} \frac{\partial}{\partial t}\left\{\gamma-\frac{p}{c^{2}} h^{00}\right\}+\frac{\partial}{\partial x^{\alpha}}\left\{\gamma \frac{u^{\alpha}}{c}+\frac{p}{c^{2}}\left\langle\frac{u^{\alpha}}{c}-h^{0 \alpha}\right)\right\}=0
$$

this may be written as

$$
\frac{1}{c}\left[\frac{\partial \gamma}{\partial t}+\bar{\nabla} \cdot(\gamma \bar{v})\right]-\frac{1}{c^{2}} \frac{\partial\left(P h^{00}\right)}{\partial t}+\frac{1}{c^{2}} \bar{\nabla} \cdot(P \bar{v})-\frac{1}{c^{2}} \bar{\nabla} \cdot\left(P h^{0}\right)=0,
$$


where $\bar{h}^{0}$ has components $h^{0 \alpha}, \alpha=1,2,3$.

Therefore

$$
\frac{\partial \gamma}{\partial t}+\bar{\nabla} \cdot(\gamma \bar{v})=-\frac{1}{c} \bar{\nabla} \cdot(P \bar{v})+\frac{1}{c^{2}}\left[\frac{\partial\left(P h^{00}\right)}{\partial t}+\bar{\nabla} \cdot\left(P \bar{h}_{0}\right)\right],
$$

so that if $h^{\nu}$ is a four-vector with components $h^{0 \nu} \equiv h^{\nu}$, then

$$
\frac{\partial \gamma}{\partial t}+\bar{\nabla} \cdot(\gamma \bar{v})=-\frac{1}{c} \bar{\nabla} P \cdot \bar{v}-\frac{P}{c} v^{\alpha},_{\alpha}+\frac{1}{c^{2}}\left(P^{\nu}\right)_{\nu} \cdot
$$

The remaining components of the divergence are given by

$$
\begin{aligned}
T^{\alpha \nu}, v & =\frac{1}{c} \frac{\partial}{\partial t}\left\{\gamma \frac{u^{\alpha}}{c}+\frac{P v^{\alpha}}{c^{3}}-\frac{p}{c^{2}} h^{\alpha 0}\right\} \\
& +\frac{\partial}{\partial x^{\beta}}\left\{\gamma \frac{v^{\alpha} v^{\beta}}{c^{2}}+\frac{p}{c^{2}}\left(\frac{v^{\alpha} v^{\beta}}{c^{2}}+\delta^{\alpha \beta}\right)-\frac{P h^{\alpha \beta}}{c^{2}}=0,\right.
\end{aligned}
$$

which may be rearranged to read

$$
\begin{aligned}
\gamma\left[\frac{\partial v^{\alpha}}{\partial t}+\bar{v} \cdot \nabla v^{\alpha}\right]= & -\frac{\partial P}{\partial x^{\alpha}}-v^{\alpha}\left[\frac{\partial \gamma}{\partial t}+\bar{\nabla} \cdot(\gamma \bar{v})\right]+\frac{1}{c} \frac{\partial\left(P h^{\alpha 0}\right)}{\partial t}+\frac{\partial\left(P h^{\alpha \beta}\right)}{\partial x^{\beta}} \\
& -\frac{1}{c^{2}}\left[\frac{\partial\left(P v^{\alpha}\right)}{\partial t}+\bar{\nabla} \cdot\left(P v^{\alpha} \bar{v}\right)\right] .
\end{aligned}
$$

If we look at the nonrelativistic limit, then, by neglecting the terms $P(v / c)$, we get

$$
\frac{\partial \gamma}{\partial t}+\bar{\nabla} \cdot(\gamma \bar{v})=\frac{1}{c^{2}}\left[\frac{1}{c} \frac{\partial\left(\mathrm{Ph}^{00}\right)}{\partial t}+\bar{\nabla} \cdot\left(\mathrm{Ph}^{-0}\right)\right] .
$$


The multiplicative factor $\frac{1}{c^{2}}$ on the right-hand side suggests that

$$
\frac{\partial \gamma}{\partial t}+\bar{\nabla} \cdot(\gamma \bar{v}) \cong 0
$$

which is the assumption we chose to place our system on a particular surface. This corresponds to a classical system where conservation of mass is assumed. Therefore, on the surface of a curve specified by

$$
T_{, \nu}^{\mu \nu}=0
$$

we must then have

$$
\gamma\left[\frac{\partial v^{\alpha}}{\partial t}+\bar{v} \cdot \bar{\nabla} v^{\alpha}\right]=-\frac{\partial P}{\partial x^{\alpha}}+\frac{1}{c} \frac{\partial\left(P h^{\alpha 0}\right)}{\partial t}+\frac{\partial\left(P h^{\alpha \beta}\right)}{\partial x^{\beta}}
$$

or

$$
\begin{aligned}
\gamma a^{\alpha} & =-\frac{\partial P}{\partial x^{\alpha}}+\frac{1}{c} \frac{\partial\left(P h^{\alpha 0}\right)}{\partial t}+\frac{\partial\left(P h^{\alpha \beta}\right)}{\partial x^{\beta}} \\
& =-\frac{\partial P}{\partial x^{\alpha}}+\frac{1}{c} \frac{\partial\left(P h^{\alpha 0}\right)}{\partial t}+\left(P h^{\alpha \beta}\right)_{\beta}, \beta=1,2,3 .
\end{aligned}
$$

Thus we may write

$$
\gamma a^{\alpha}=\tau^{\alpha \beta}, \beta
$$

where $\tau^{\alpha \beta}=-P \hat{g}^{\alpha \beta}+P h^{\alpha \beta}=-P\left(\hat{g}^{\alpha \beta}-h^{\alpha \beta}\right)$.

The term $\frac{1}{c} \frac{\partial\left(\mathrm{Ph}^{\alpha 0}\right)}{\partial t}$ has been neglected in (175). 
Thus we see that the geometric character of the hypersurface, contained in the term $\mathrm{Ph}^{\alpha \beta}$, behaves as if it were a viscous effect to be added to the normal viscous effects. Recalling Eq. (165), it may be seen that the viscous-like effects of the geometry of the hypersurface depend upon the density gradient. If these terms exist, they must be very small in everyday phenomena. Yet if we consider phenomenon which involve very large density gradients, these terms could become large enough to see.

4. Shock Waves. One field of physical phenomena that displays large density gradients is shock waves. Therefore let us take a quick look at the effect of these additional terms on the description of a shock front for a steady, onedimensional shock.

The total stress in a steady, one-dimensional shock would be given by

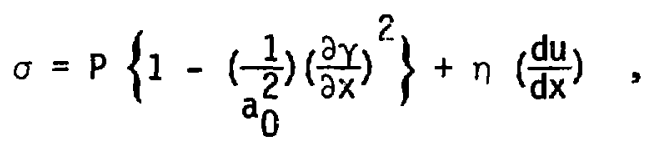

when $\hat{g}_{11}=1$ and $h_{11}$ is evaluated using Eq. (165). However, for a steady shock we also have

$$
\begin{aligned}
\gamma u & =k_{1}, \\
k_{1} u+\sigma & =k_{2},
\end{aligned}
$$

and

$$
k_{1}^{2} E-\frac{\sigma^{2}}{2}=k_{3}
$$

These equations represent the conservation of mass, momentum, and energy. By using the conservation of mass relation we may write the total stress as

$$
\sigma=P+\eta_{e f f}\left(\frac{d u}{d x}\right) \text {, }
$$


where

$$
\eta_{e f f} \equiv n-\frac{P k_{1}^{2}}{a_{0}^{2} u^{4}}\left(\frac{d u}{d x}\right)
$$

may be called the effective viscous coefficient. Since within the shock front the velocity gradient $d u / d x$ is negative, we see that the effective viscolis coefficient acts so as to thicken the shock front when compared to the classical viscous coefficient $n$.

\section{E. Mass Conservative Electrodynamics}

One of the incentives for seeking to determine whether the five equations of motion were generalizations of the classical hydrodynamic equations was the possibility of shedding new light upon fusion plasmas. Now before mass conversion is accomplished the plasma must reach certain conditions. The attainment of these conditions involve electromagnetic fields not encountered in usual circumstances on earth. If the Dynamic Theory is to be believed, then perhaps it may provide new insight into the attainment of the appropriate conditions before mass conversion begins.

The following development still assumes conservation of mass in order to see the geometry of the hypersurface for a system under the influence of electromagnetic fields.

Suppose we now describe the behavior of charged matter under the influence of an electromagnetic field from the viewpoint of the Dynamic Theory. From this viewpoint the conservation of mass has the effect of restricting our system to a four-dimensional hypersurface which is embedded in the five-dimensional manifold of space, time, and mass density.

Since we desire to consider the effects of an electromagnetic field we must consider a gauge function. When a gauge function exists, the square of the arc length in the entropy space is related to the square of the arc length in the sigma space by

$$
\left(d q^{0}\right)^{2}=\hat{g}_{i j} d x^{i} d x^{j}=\left(\frac{1}{h_{00}}\right) \hat{g}_{i j} d x^{i} d x^{j}=\left(\frac{1}{h_{00}}\right)(d \sigma)^{2} .
$$


When the system is restricted to a hypersurface by the relation $x^{4}=$ $x^{4}\left(x^{0}, x^{1}, x^{2}, x^{3}\right)$, then the entropy surface may be written as

$$
\left(d q^{0}\right)^{2}=\hat{a}_{\alpha \beta} d u^{\alpha} d u^{\beta},
$$

where

$$
\hat{a}_{\alpha \beta}=\hat{g}_{i j} \frac{\partial x^{i}}{\partial u^{\alpha}} \frac{\partial x^{j}}{\partial u^{\beta}}=g_{i j} x_{\alpha}^{i} x_{\beta}^{j} .
$$

Likewise for the sigma surface

$$
(d \sigma)^{2}=\hat{\hat{a}}_{\alpha \beta} d u^{\alpha} d u^{\beta}
$$

where

$$
\hat{\hat{a}}_{\alpha \beta}=\hat{\hat{g}}_{i j} x_{\alpha}^{i} x_{\beta}^{j} .
$$

Thus we have

$$
\hat{a}_{\alpha \beta}=\left(\frac{1}{h_{00}}\right) \hat{a}_{\alpha \beta} \text {. }
$$

The principle of increasing entropy requires that the equations of motion be geodesics in the entropy space but they will appear as equations involving forces in the sigma space. We desire to expose these forces and therefore should work in the sigma space. Our objective then is to determine the effect of embedding a four-dimensional surface given by $x^{4}=x^{4}\left(x^{0}, x^{1}, x^{2}, x^{3}\right)$ in the sigma space and thus obtain a sigma surface describing a system subjected to the classical conservation of mass restriction.

Having previously determined the metric coefficients for the entropy space by Eqs. (163) and (164) we may write the coefficients for the sigma surface as 


$$
\hat{a}_{\alpha \beta}=h_{00} \hat{a}_{\alpha \beta}=h_{00}\left[\hat{g}_{\alpha \beta}+h_{\alpha \beta}\right] \text {. }
$$

However by considering the effects of the electromagnetic field as a force we must first consider the space field tensor:

$$
F_{i j}=\left|\begin{array}{ccccc}
0 & E_{1} & E_{2} & E_{3} & v_{0} \\
-E_{1} & 0 & B_{3} & -B_{2} & V_{1} \\
-E_{2} & -B_{3} & 0 & B_{1} & V_{2} \\
-E_{3} & B_{2} & -B_{1} & 0 & V_{3} \\
-V_{0} & -V_{1} & -V_{2} & -V_{3} & 0
\end{array}\right| .
$$

If we restrict ourselves to the classical field quantities $\bar{E}$ and $\bar{B}$ and for the moment assume that the field quantities $V_{0}$ and $v$ are zero, then we obtain only the effects of the hypersurface viewpoint. This assumption seems reasonable considering the possible interpretation of the new field quantities in terms of nuclear effects. Under this assumption our field tensor becomes

$$
F_{i j}=\left|\begin{array}{ccccc}
0 & E_{1} & E_{2} & E_{3} & 0 \\
-E_{1} & 0 & B_{3} & -B_{2} & 0 \\
-E_{2} & -B_{3} & 0 & B_{1} & 0 \\
-E_{3} & B_{2} & -B_{1} & 0 & 0 \\
0 & 0 & 0 & 0 & 0
\end{array}\right| .
$$

We can now use this space field tensor to determine the appearance of the fields when viewed from the surface. The surface field tensor will be given by

$$
F_{\alpha \beta}=F_{i j} x_{\alpha}^{i} x_{\beta}^{j}
$$

152 
But since $x_{\alpha}^{i}=\delta_{\alpha}^{i}$ for $i, \alpha=0,1,2,3$ and $x_{\alpha}^{4}=f_{\alpha}$, the surface field tensor of a purely electromagnetic space field tensor is only the four-dimensional portion of the space field tensor since $F_{j 4}=0$ for $i=0,1,2,3,4$.

Thus when we use the relativistic energy-momentum tensor for the surface, we have

$$
T^{\mu \nu}=\gamma u^{\mu} u^{\nu}+\frac{1}{c^{2}}\left[F_{\alpha}^{\mu} F^{\alpha \nu}+\frac{1}{4} \hat{a}^{\mu \nu} F^{\alpha \beta} F_{\alpha \beta}\right],
$$

which is the relativistic energy-momentum tensor for matter under the influence of electromagnetic fields. But since $\hat{a}_{\alpha \beta}=\hat{g}_{\alpha \beta}+h_{\alpha \beta}$, then (176) becomes

$$
T^{\mu \nu}=\gamma u^{\mu} u^{\nu}+\frac{1}{c^{2}}\left[F_{\alpha}^{\mu} F^{\alpha \nu}+\frac{1}{4}\left(\hat{g}^{\mu \nu}+h^{\mu \nu}\right) F^{\alpha \beta} F_{\alpha \beta}\right]
$$

or

$$
T^{\mu \nu}=T_{\text {rel }}^{\mu \nu}+T_{\text {geo }}^{\mu \nu}
$$

where

$$
T_{r e 1}^{\mu \nu} \equiv \gamma u^{\mu} u^{\nu}+\frac{1}{c^{2}}\left[F_{\alpha}^{\mu} F^{\alpha \nu}+\frac{1}{4} \hat{g}^{\mu \nu \nu} F^{\alpha \beta} F_{\alpha \beta}\right]
$$

is the four-dimensional space relativistic energy momentum tensor and

$$
T_{g e 0}^{\mu \nu} \equiv\left(\frac{1}{4 c^{2}}\right) h^{\mu \nu} F^{\alpha \beta} F_{\alpha \beta}
$$

is the portion of the energy-momentum tensor which contains the geometrical properties of the hypersurface.

From Eq. (177) we can say that the Dynamic Theory has the appearance of adding a term to the relativistic energy-momentum tensor. This term contains 
the geometrical character of the surface and represents the difference between the appearance of the energy-momentum tensor when viewed from the surrounding space as compared to the view from the hypersurface.

If we take the divergence of the energy-momentum tensor (177), we have

$$
T_{, \nu}^{\mu \nu}=T_{\text {rel }, \nu}^{\mu \nu}+T_{\text {geo }, \nu}^{\mu \nu} .
$$

The additional force terms from the surface geometry are given by

$$
\left(\frac{1}{4 c^{2}}\right)\left(h^{\mu \nu} F^{\alpha \beta} F_{\alpha \beta}\right), \nu=F^{\mu}
$$

But if we define

$$
F^{\alpha \beta} F_{\alpha \beta} \equiv-16 \tau \xi
$$

as the electromagnetic energy density, where

$$
\xi=\frac{1}{8 \pi}\left(E^{2}+B^{2}\right)
$$

then the geometric energy-momentum tensor becomes

$$
T_{\text {geo }}^{\mu \nu}=\frac{-4 \pi h^{\mu \nu} \xi}{c^{2}}
$$

and the additional forces are given by

$$
F^{\mu}=-\frac{4 \pi}{c^{2}}\left(h^{\mu \nu} \xi\right), \nu:
$$

We may also look at the radiation pressure predicted by the Dynamic Theory to see how the surface restriction affects the relativistic prediction of radiation pressure. 
The relativistic radiation pressure is taken as one third of the threedimensional Maxwell stress tensor which is the space portion of the energymomentum tensor, or

$$
T_{\alpha \beta}^{M}=\frac{1}{4 \pi}\left(E_{\alpha \beta} E_{\beta}+B_{\alpha \beta} B_{\beta}\right)-\delta_{\alpha \beta} \xi
$$

where $\alpha, \beta=1,2,3$.

To get the equivalent stress tensor for the Dynamic radiation pressure we must add the space portion of (178) so that the total stress tensor becomes

$$
\begin{aligned}
T_{\alpha \beta} & =\frac{1}{4 \pi}\left(E_{\alpha \beta} E_{\beta}+B_{\alpha \beta} B_{\beta}\right)-\delta_{\alpha \beta} \xi-4 h_{\alpha \beta} \xi \\
& =\frac{1}{4 \pi}\left(E_{\alpha} E_{\beta}+B_{\alpha \beta} B_{\beta}\right)-\xi\left(\delta_{\alpha \beta}+h_{\alpha \beta}\right) .
\end{aligned}
$$

We can then obtain the negative of the trace by

$$
\begin{aligned}
-\{T\} & =-\left[\frac{1}{4 \pi}\left(E^{2}+B^{2}\right)-3 \xi-\left(h_{11}+h_{22}+h_{33}\right) \xi\right] \\
& =-\left[-\xi-\left(h_{11}+h_{22}+h_{33}\right) \xi\right] .
\end{aligned}
$$

The radiation pressure is then given by

$$
P=\frac{\xi}{3}\left[1+h_{11}+h_{22}+h_{33}\right] \text {. }
$$

The first term in (179) is the classical radiation pressure in electrodynamics. The remaining three terms give the difference between the pressure predicted by the Dynamic Theory and the classical prediction. To determine what this difference is let us restrict our system to again be very near equilibrium so that the $\hat{g}_{\alpha 4}=0$ for $\alpha=0,1,2,3$ and $\hat{g}_{44}=$ constant. Thus we have a flat space. For this space the 


$$
h_{\alpha \alpha}=\frac{\hat{g}_{44}}{a_{0}^{2}}\left(\frac{\partial \gamma}{\partial x^{\alpha}}\right)^{2}
$$

from (163) and $\hat{g}_{44}=-1$. Thus

$$
h_{11}+h_{22}+h_{33}=-\frac{1}{a_{0}^{2}}\left[\left(\frac{\partial \gamma}{\partial x}\right)^{2}+\left(\frac{\partial \gamma}{\partial x^{3}}\right)^{2}+\left(\frac{\partial \gamma}{\partial x^{3}}\right)^{2}\right] .
$$

By substituting (180) into (179) the pressure becomes

$$
P=\frac{\xi}{3}\left\{1-\frac{1}{a_{0}^{2}}\left[\left(\frac{\partial \gamma}{\partial x}\right)^{2}+\left(\frac{\partial \gamma}{\partial x^{2}}\right)+\left(\frac{\partial \gamma}{\partial x^{3}}\right)^{2}\right]\right\}
$$

However, since the classical pressure is given by $P_{C}=\frac{\xi}{3}$, then the pressure predicted by the Dynamic Theory becomes

$$
P_{D}=P_{C}\left\{1-\left(\frac{1}{a_{0}^{2}}\right)\left[\left(\frac{\partial \gamma}{\partial x^{1}}\right)^{2}+\left(\frac{\partial \gamma}{\partial x^{2}}\right)^{2}+\left(\frac{\partial \gamma}{\partial x^{3}}\right)^{2}\right]\right\} \text {. }
$$

We see then that the Dynamic Theory predicts a decrease in the radiation pressure as a result of viewing the system to be restrictea to a four-dimensional hypersurface embedded in a five-dimensional space. The amount of this decrease in pressure depends upon the gradient of the mass density and the constant $a_{0}$. Once the constant $a_{0}$ is determined, then the deviation in predicted pressures can be specified.

\section{F. Field Equations}

Einstein's General Theory of Relativity made possible various models of the universe through solutions of his field equations. These solutions gave the metric coefficients according to the various models considered. Using Weyl's interpretations the Dynamic Theory leads to a five-dimensional set of field equations. These equations are generalizations of Einstein's field equations and, together with their boundary conditions will be presented here. 
1. Nonlinear Field Equations. The seven Maxwell equat:ons may be used to determine the gaugs function for the five-dimensional manifold for an isolated system. The remaining metric coefficients may be determined by Einstein-type field equations. Therefore let us assume that the gauge function is simply a constant so that the following may concentrate upon the remaining coefficients.

We shall suppose that the metric coefficients are determined by the gravitating effects of mass as is done in Einstein's Theory and, further, if our system is infinitely far from grayitating matter, then it will be in an equilibrium state. Therefore the boundary conditions to be imposed is that the system satisfy the conditions of equilibrium when far removed from gravitating matter. In equilibrium the secord partial derivatives will be constants evaluated at this equilibrium state. Therefore the limiting metric will be one with constant coefficients. This is similar to the conditions used in the Schwarzchild solution to Einstein's equations.

Thus our limiting metric will be

$$
\left(d q^{0}\right)^{2}=c^{2} d t^{2}-\left(d x^{\alpha}\right)^{2}, \alpha=1,2,3,4 .
$$

The field equations in a vacuum are

$$
R_{i j}=0
$$

or

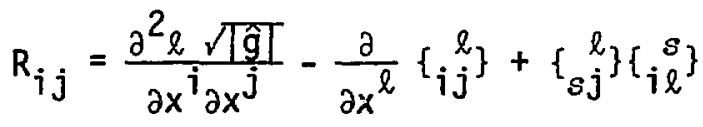

$$
\begin{aligned}
& -\left\{\begin{array}{c}
s, j \\
\}
\end{array} \frac{\partial \ln \sqrt{g}[}{\partial x^{s}}\right.
\end{aligned}
$$

where $i, j=0,1,2,3,4$.

Setting $c=1$ and taking the trajectory to be a straight line far from gravitating matter leads us to assume the limiting form of the metric to be 


$$
\left(d q^{0}\right)^{2}=(d t)^{2}-(d r)^{2}-r^{2}(d \theta)^{2}-r^{2} \sin ^{2} \partial(d \phi)^{2}-\frac{(d r)^{2}}{a_{0}^{2}} .
$$

(Note: This involves an assumption concerning the nature of how the mass density is affected and should be considered in detail later.)

We may further set $a_{0}=1$ in the same sense as $c=1$ for further simplification. Therefore let us adopt the form

$$
\begin{aligned}
\left(d q^{0}\right)^{2} & =f_{1}(\bar{r}, \gamma)(d t)^{2}-f_{2}(\bar{r}, \gamma)(d r)^{2}-r^{2}(d \theta)^{2}-r^{2} \sin ^{2} \theta(d \phi)^{2} \\
& -f_{3}(\bar{r}, \gamma)(d r)^{2} .
\end{aligned}
$$

Here the cross-product terms have been rejected due tu arguments of static spherical symmetry and simplicity.

Suppose for the purpose of finding the functions $f_{1}, f_{2}$. and $f_{3}$ we follow Schwarzchild's example by setting

$$
f_{1}=e^{\mu} ; f_{2}-e^{\lambda} ; f_{3}=e^{\nu} \text {. }
$$

Substituting these into the metric of (182), the metric becomes

$$
\left(d q^{0}\right)^{2}=-e^{\lambda}(d r)^{2}-r^{2}(d \theta)^{2}-r^{2} \sin ^{2} \theta(d \phi)^{2}-e^{\nu}(d \gamma)^{2}+e^{\mu}(d t)^{2} .
$$

Thus the metric coefficients are given by

$$
\begin{aligned}
& \hat{g}_{00}=e^{\mu}, \hat{g}_{11}=-e^{\lambda}, \hat{g}_{22}=-r^{2}, \hat{g}_{33}=-r^{2} \sin ^{2} \theta, \\
& \hat{g}_{44}=-e^{\nu}, \text { and } \hat{g}_{i j}=0 \text { for } i \neq j .
\end{aligned}
$$

The determinant for the quadratic form of Eq. (183) is

$$
\hat{g}=\hat{g}_{00} \hat{g}_{11} \hat{g}_{22} \hat{g}_{33} \hat{g}_{44}=e^{(\lambda+\mu+\nu)} r^{4} \sin ^{-2} \theta
$$


and the contravariant tensor $\hat{\mathrm{g}}^{\mathrm{ij}}$ is given by the matrix

$$
\hat{g}^{i j}=\left(\begin{array}{ccccc}
e^{-\mu} & 0 & 0 & 0 & 0 \\
0 & -e^{-\lambda} & 0 & 0 & 0 \\
0 & 0 & -\frac{1}{r^{2}} & 0 & 0 \\
0 & 0 & 0 & \frac{1}{r^{2} \sin ^{2} \theta} & 0 \\
0 & 0 & 0 & 0 & -e^{-\nu}
\end{array}\right)
$$

The Christoffel symbols $\left\{_{i j}^{\ell}\right.$ \} may now be constructed and since $\hat{g}_{i j}=0$ when $i \neq j$ we have

$$
\left\{_{i j}^{\ell}\right\}=\left(\frac{1}{2}\right) \hat{g}^{\ell \ell}\left(\frac{\partial \hat{g}_{i \ell}}{\partial x^{j}}+\frac{\partial \hat{g}_{j \ell}}{\partial x^{j}}-\frac{\partial \hat{g}_{i j}}{\partial x^{l}}\right) \text {. }
$$

The non-zero Christoffel symbols are then

$$
\begin{aligned}
& \left\{\begin{array}{c}
0 \\
0
\end{array}\right\}=\frac{1}{2}\left(\frac{\partial \mu}{\partial r}\right), \quad\left\{\begin{array}{c}
0 \\
04
\end{array}\right\}=\frac{1}{2}\left(\frac{\partial \mu}{\partial \gamma}\right), \quad\left\{\begin{array}{c}
1 \\
00
\end{array}\right\}=\frac{1}{2}\left(\frac{\partial \mu}{\partial r}\right) e^{(\mu-\lambda)}, \\
& \left\{{ }_{11}^{1}\right\}=\frac{1}{2}\left(\frac{\partial \lambda}{\partial r}\right), \quad\left\{{ }_{14}^{1}\right\}=\frac{1}{2}\left(\frac{\partial \lambda}{\partial \gamma}\right), \quad\left\{{ }_{22}^{1}\right\}=-r e^{-\lambda}, \\
& \left\{{ }_{33}^{1}\right\}=-r e^{-\lambda} \sin ^{2} \theta, \quad\left\{{ }_{44}^{1}\right\}=-\frac{1}{2}\left(\frac{\partial \nu}{\partial r}\right) e^{(\nu-\lambda)}, \quad\left\{{ }_{21}^{1}\right\}=\frac{1}{r}, \\
& \left\{\left\{_{33}^{2}\right\}=-\sin \theta \cos \theta, \quad\left\{{ }_{31}^{3}\right\}=\frac{1}{r}, \quad\left\{{ }_{32}^{3}\right\}=\cot \theta=\frac{\cos \theta}{\sin \theta},\right. \\
& \left.\left.{ }_{00} \begin{array}{c}
4 \\
0
\end{array}\right\}=\frac{1}{2}\left(\frac{\partial \mu}{\partial \gamma}\right) e^{(\mu-\nu)}, \quad{ }^{4}{ }_{11}^{4}\right\}=-\frac{1}{2}\left(\frac{\partial \lambda}{\partial \gamma}\right) e^{(\lambda-\nu)}, \\
& \left\{\begin{array}{c}
4 \\
4
\end{array}\right\}=\frac{1}{2}\left(\frac{\partial v}{\partial r}\right) \text {, and }\left\{\begin{array}{c}
4 \\
44
\end{array}\right\}=\frac{1}{2}\left(\frac{\partial v}{\partial \gamma}\right) \text {. }
\end{aligned}
$$

159 
The equations for $\mu, \nu$, and $\lambda$ are then obtained by inserting the Christoffe 1 symbols into Eq. (181) and setting $R_{i j}=0$. This procedure yields five equations:

$$
\begin{aligned}
& {\left[-\frac{1}{2}\left(\frac{\partial^{2} \mu}{\partial r^{2}}\right)+\frac{1}{4}\left(\frac{\partial \mu}{\partial r}\right)\left(\frac{\partial \lambda}{\partial r}\right)-\frac{1}{4}\left(\frac{\partial \mu}{\partial r}\right)^{2}-\frac{1}{4}\left(\frac{\partial \mu}{\partial r}\right)\left(\frac{\partial \nu}{\partial r}\right)-\frac{1}{r}\left(\frac{\partial \mu}{\partial r}\right)\right] e^{(\mu-\lambda)}} \\
& +\left[-\frac{1}{2}\left(\frac{\partial^{2} \mu}{\partial \nu^{2}}\right)+\frac{1}{4}\left(\frac{\partial \mu}{\partial \gamma}\right)\left(\frac{\partial \nu}{\partial \gamma}\right)-\frac{1}{4}\left(\frac{\partial \mu}{\partial \gamma}\right)^{2}-\frac{1}{4}\left(\frac{\partial \mu}{\partial \gamma}\right)\left(\frac{\partial \lambda}{\partial \gamma}\right)\right] e^{(\mu-\nu)}=0 ; \\
& \frac{1}{2}\left(\frac{\partial^{2} \mu}{\partial r^{2}}\right)-\frac{1}{4}\left(\frac{\partial \lambda}{\partial r}\right)\left(\frac{\partial \mu}{\partial r}\right)+\frac{1}{4}\left(\frac{\partial \mu}{\partial r}\right)^{2}-\frac{1}{r}\left(\frac{\partial \lambda}{\partial r}\right)-\frac{1}{4}\left(\frac{\partial \lambda}{\partial r}\right)\left(\frac{\partial v}{\partial r}\right)+\frac{1}{4}\left(\frac{\partial v}{\partial r}\right)^{2}+\frac{1}{2}\left(\frac{\partial^{2} v}{\partial r^{2}}\right) \\
& +\left[\frac{1}{2}\left(\frac{\partial^{2} \lambda}{\partial \gamma^{2}}\right)+\frac{1}{4}\left(\frac{\partial \lambda}{\partial \gamma}\right)^{2}+\frac{1}{4}\left(\frac{\partial \lambda}{\partial \gamma}\right)\left(\frac{\partial \mu}{\partial \gamma}\right)-\frac{1}{4}\left(\frac{\partial \lambda}{\partial \gamma}\right)\left(\frac{\partial \nu}{\partial \gamma}\right)\right] e^{(\lambda-\nu)}=0 ; \\
& e^{-\lambda}\left[1+\left(\frac{1}{2}\right) r\left(\frac{\partial \mu}{\partial r}+\frac{\partial \nu}{\partial r}-\frac{\partial \lambda}{\partial r}\right)\right]-1=0 \text {; } \\
& \sin ^{2} \theta\left\{e^{-\lambda}\left[1+\left(\frac{1}{2}\right) r\left(\frac{\partial \mu}{\partial r}+\frac{\partial \nu}{\partial r}-\frac{\partial \lambda}{\partial r}\right)\right]-1\right\}=0 ; \quad \text { and } \\
& \frac{1}{2}\left(\frac{\partial^{2} \mu}{\partial \gamma^{2}}\right)+\frac{1}{2}\left(\frac{\partial^{2} \lambda}{\partial \gamma^{2}}\right)+\frac{1}{4}\left(\frac{\partial \lambda}{\partial \gamma}\right)^{2}-\frac{1}{4}\left(\frac{\partial \lambda}{\partial \gamma}\right)\left(\frac{\partial \nu}{\partial \gamma}\right)-\frac{1}{4}\left(\frac{\partial \nu}{\partial \gamma}\right)\left(\frac{\partial \mu}{\partial \gamma}\right)+\frac{1}{4}\left(\frac{\partial \mu}{\partial \gamma}\right)^{2} \\
& +\mathrm{e}^{(\nu-\lambda)}\left[\frac{1}{r}\left(\frac{\partial \nu}{\partial r}\right)+\frac{1}{2}\left(\frac{\partial^{2} v}{\partial r^{2}}\right)-\frac{1}{4}\left(\frac{\partial \nu}{\partial r}\right)\left(\frac{\partial \lambda}{\partial r}\right)+\frac{1}{4}\left(\frac{\partial v}{\partial r}\right)\left(\frac{\partial \mu}{\partial r}\right)+\frac{1}{4}\left(\frac{\partial \nu}{\partial r}\right)^{2}\right]=0 .
\end{aligned}
$$

Equation (185) is a repetition of Eq. (184). Therefore there are only four equations on the three functions $\lambda, \mu$, and $\nu$ to consider. This situation is similar to the one in Schwarzchild's solution. It is relatively easy to show that these equations reduce to Einstein's equations of Schwarzchild.

Here the solution to these equations are not obtained. However, obtaining the solution will provide a model of the universe in which mass conversion, or 
creation, plays a free role as an independent coordinate. This picture of the universe should produce an interesting and perhaps enlightening view of black holes, parijcularly since black holes involve tremendous mass densities and mass density changes.

2. Linearization of Field Equations. The set of differential equations for the functions $\mu, \nu$, and $\lambda$ given in the preceding section are nonlinear equations which may prove difficult to solve. Linearizations of these equations may provide approximate solutions that could prove useful.

The procedure to linearize the equations is identical to the process of linearizing the general relativistic field equations. However, here the equations involve five-dimensions and we therefore obtain 15 partial differential equations.

If we restrict our system to be sufficiently near an equilibrium state so that the metric tensor differs only slightly from the flat-space metric for the equilibrium state, then we may write the metric tensor as the flat-space tensor $n_{i j}$ given by

$$
\eta_{i j}=\left(\begin{array}{rrrrr}
-1 & 0 & 0 & 0 & 0 \\
0 & -1 & 0 & 0 & 0 \\
0 & 0 & -1 & 0 & 0 \\
0 & 0 & 0 & -1 & 0 \\
0 & 0 & 0 & 0 & -1
\end{array}\right) .
$$

Where the five-dimensional Minkowski coordinates ict, $x, y, z, \frac{\gamma}{a_{0}}$ are used for simp1icity, plus a perturbation term $\varepsilon \xi_{i j}$ so that

$$
\hat{g}_{i j}=\eta_{i j}+\varepsilon \xi_{i j}, i, j=0,1,2,3,4,
$$

then only the first-order terms in the parameter $\varepsilon$ are to be considered as significant in all equations.

By following the same procedures as in the relativistic case the linearized partial differential equations for the 15 distinct perturbation terms $\gamma_{i j}$ may be found to be 


$$
\frac{\partial^{2}}{\partial x^{j} \partial x^{j}} \sum_{\ell=0}^{4} \xi_{\ell \ell}-\sum_{\ell=0}^{4} \frac{\partial}{\partial x^{l}}\left[\frac{\partial \xi_{\ell j}}{\partial x^{j}}+\frac{\partial \xi_{l j}}{\partial x^{j}}-\frac{\partial \xi_{i j}}{\partial x^{l}}\right]=0 \text {. }
$$

The only difference between Eqs. (186) and the linearized relativistic equations is the increased dimensionality which results in summing over the five coordinates and allowing the indices to range over five dimensions instead of the four used in the general relativistic approach.

\section{CONCLUSIONS}

The generality of the fundamental laws adopted by the Dynamic Theory makes it possible to arrive at a great number of conclusions. However, only a few will be selected for discussion here.

The first question which provided the motivation to seek a new theoretical approach to physics concerned the uniqueness of the velocity of light as a 1 imiting velocity for all natural forces. The answer is provided by the axiomatic development of the second law. This development produced an integrating factor for the differential statement of the first law. A rharacteristic velocity was shown to exist in the definition of the absolute velocity. That absolute velocity is given by a constant velocity process at which the integrating factor is zero. The important point in the development which provides the answer to the uniqueness of the velocity is the proof that the integrating factor is independent of the nature of the force. Therefore, if the absolute velocity is independent of the force, it must be applicable to all forces and hence unique.

Since by definition the absolute velocity is a constant in one reference frame, it must also be a constant in any other reference frame moving with a constant velocity relative to the first. Thus the absolute velocity must be unique and a constant in all reference frames moving with constant relative velocities. The experimental and theoretical evidence of electromagnetism requires that this absolute velocity be the same value in all these reference frames. This requirement leads to the princip'? of Lorentz covariance. Then al1 the laws of nature must be Lorentz covariant whether electromagnetic, gravitational, or nuclear since the absolute velocity is unique and independent of the force. 
He find that the appearance of the integrating factor also clarifies the relationship between the velocity dependent relativistic mass and velocity dependent forces. In Einstein's Special Theory of Relativity we find it necessary to consider a velocity dependent relativistic mass and a velocity independent force. However, in the Dynamic Theory there appear two differential expressions which become important for any system description. The first differential expression is the first law itself, which, in simplified form is

$$
\bar{d} E=m \dot{q} d \dot{q}-\sqrt{1-\dot{q}^{2} / c^{2}} F(q) d q .
$$

The other is the expression for the differential change in mechanical entropy or

$$
\mathrm{d} S=\frac{\mathrm{m} \dot{\mathrm{q}} \mathrm{dq}}{\sqrt{1-\dot{\mathrm{q}}^{2} / \mathrm{c}^{2}}}-\mathrm{F}(\mathrm{q}) \mathrm{dq} .
$$

The integral of $\overline{\mathrm{d}} E$ depends upon the path and therefore is of little utility in determining the actual process, or path, taken by the system. On the other hand, the entropy is independent of the path. This characteristic together with the principle of increasing entropy for an isolated system establishes the variational principle for determining the path. Since the objective of dynamics is to find the path taken, then it becomes obvious that the differential expression for the entropy change plays a dominant role and it is in this expression that we find Einstein's velocity dependent relativistic mass and a velocity independent force. However, from the Dynamic Theory's point of view the first law describes "reality" and here we find velocity dependent forces and mass that are independent of the velocity.

Thus we find that the Dynamic Theory requires that the kinetic energy, which comes from the energy expression, to be the classical kinetic energy, or $\left(\frac{1}{2}\right) \mathrm{mq}^{2}$, while from the entropy expression comes a function, $-m c^{2} \sqrt{1-\dot{q}^{2} / c^{2}}$, which may be called the "kinetic entropy." In this manner the Dynamic Theory clarifies the mental controversy which motivated it. For from this viewpoint kinetic energy does not depend upon the value of the limiting velocity; kinetic entropy does. The two are distinct concepts, however, the distinction can not be seen from a relativistic point of view. 
Though the philosophy which formed the basis for the development of the Dynamic Theory made it necessary to set aside the fundamental postulates of Einstein's relativistic theories and Newtonian laws and begin to establish a new theoretical basis, we find that the Dynamic Theory requires the same conclusions as special cases. Therefore the viewpoint of the Dynamic Theory, which appeared incompatible with current theories, not only supports current theories but lends them additional strength from its more general approach. The concept of a limiting velocity takes on a new, more fundamental, character as the mechanical counterpart to thermodynami:'s absolute zero temperature. Yet because of the different point of view this limiting velocity does not have the absolute character attributed to it in relativistic theory for we find that its value depends upon the dimensionality of the system.

When the metric provided by the stability conditions is considered, we find that the Dynamic Theory, through the second law, specifies the geometry which must be considered. This removes the necessity of assuming a particular geometric character and, for an isolated system specifies the type of geometry which can satisfy the principle of increasing entropy. Using Weyl's interpretations from his unified field theory we find that the Dynamic Theory answers some questions that Weyl's theory leaves unanswered or introduces.

First recal1 that Weyl assumed the existence of a quadratic and linear differential form. The Dynamic Theory produces two quadratic, plus a linear, differential forms for isolated systems and it is the interplay of these three forms which provide the answers. Weyl's theory raised a question concerning integrability and Einstein produced an argument which indicated that Weyl's theory would not produce the sharp spectral lines we see from atoms changing states. Weyl only had one quadratic form. The Dynamic Theory has two; one an entropy manifold, which yields an integrable arc length of entropy, the other manifold is related to it by the gauge function. Thus the second law requires that the entropy manifold have an integrable arc length though the other may not and in that event the gauge function behaves like an integrating factor.

An additional feature of the interrelation of the three differential forms lies in its ability to shed new light upon the existence of both positive and negative electromagnetic charges. Weyl's theory, as well as Maxwell's leaves this question unanswered. Weyi defined the potentials $\phi_{k}$, which are the coefficients of the linear differential form, as 


$$
\phi_{k}=\frac{1}{2} \frac{\partial \ln f}{\partial x^{k}}
$$

where $f$ is the gauge function. Though the gauge function relates Heyl's "distance measures" $\hat{\ell}$ and $\ell$ by

$$
\hat{\ell}^{2}=f \ell^{2},
$$

Weyl did not take the potentials to be logarithmic derivatives of a radical function. As a result, he pointed out that one way of accounting for the difference between positive and negative electricity would be to introduce a radical somewhere.

The Dynamic Theory requires that the entropy manifold be related to the sigma manifold by

$$
\left(d q^{0}\right)^{2}=f(d \sigma)^{2}
$$

Here the principle of increasing entropy provides the variational principle hence there is added significance in considering the differential change in entropy or

$$
d q^{0}=f^{1 / 2} d \sigma
$$

Thus the only mathematically consistent definition for $\phi_{k}$ is

$$
\phi_{k}= \pm \frac{1}{2} \frac{\partial \ln f}{\partial x^{k}}
$$

thereby accounting for the existence of both positive and negative electromagnetic potentials.

The recognition of the existence of the radical together with the fact that the second law demands a real function for the entropy since $d q^{0} \geq 0$ for an isolated system provides the capability to remove London's difficulty with imaginary distances in his quantization of Weyl's theory. This coupled with the 
logical demand, by the Dynamic Theory, for the conditions resulting in quantization demonstrate the power of the theory to unify the whole of physical theory under the umbrella of a single set of generalized laws.

The real power of the theory begins to make its appearance when some of the restrictions are relaxed. This is demonstrated here by considering a thermodynamic work term in the first law together with three mechanical work terms. For it is here that forces with the appearance of nuclear forces appears. These forces come from the action of new field quantities appearing as the fifthdimensional components of the gauge field. Though these field components first appear in an interrelationship with the classical electromagnetic fields it is the quantization that provides the connection of the field quantities with nuclear properties. The connection shows up first in the prediction of anomalous magnetic moments but gains additional support from the appearance of three spin vectors that become necessary to complete a set of commuting observables.

Thus the Dynamic Theory offers the hope of including nuclear theory and elementary particle theory within the same unified structure. Two aspects from this approach seem particularly significant in their support of this contention. One is the appearance of octets as the number of allowed combinations of fundamental eigenvalues for the components of the three spin vectors. The other is the necessity for one of the vectors to be a four-dimensional spin vector instead of being a three-dimensional one. Could this not be the additional freedom necessary to include the newly discovered elementary particles within the same theoretical framework as the others?

When mass conservative systems are considered, the theory offers new views of several different physical phenomena. First, there is the viscous effect of the geometry of a four-dimensional hypersurface embedded by mass conservation into the five-dimensional manifold. This allows the prediction of thickened shock fronts.

Another phenomenon which appears under a new light in the Dynamic Theory is the electromagnetic containment of an ionized plasma. This should prove to be of considerable benefit when applied to a fusion reactor. For here the theory gives a geumetric view of a hypersurface embedded into a five-dimensional space until such time as mass conversion begins to take place then the system description becomes one given by the five equations of motion in the full fivedimensiona 1 space. 
It is here that the concept of limiting rate of mass conversion becomes prominent. This limiting rate stands as a fundamental concept with the same character as the speed of light in relativistic theories and within this theory where mass conservation applies. As far as is known, the prediction of the existence of a limiting rate of mass conversion is original. However, the manner in which it appears within the theory is identical with the manner in which the limiting, or absolute, velocity appears, namely as the result of applying the third law. Further, this coincides with the classical appearance of the absolute zero temperature as a limiting value. Thus from the Dynamic Theory's point of view the concept of a limiting rate of mass conversion is just as fundamental as the concept of a limiting temperature or velocity.

On the other hand the existerice of a limiting rate of mass conversion seems intuitively pleasing. For if we accept the notion that something cannot travel with infinite velocity, it seems only reasonable to believe that mass may not be converted instantaneously.

The third law establishes a limiting value of the time rate of change of one of the coordinates, or variables, by the integrating factor going to zero. Then for a system near equilibrium, so that a flat space may be considered, the integrating factor goes to zero when

$$
1=\frac{v^{2}}{c^{2}}+\frac{\dot{\gamma}^{2}}{a_{0}^{2} c^{2}}
$$

where $\dot{\gamma} \equiv d \gamma / d t$ and $v^{2}=\left(\frac{d x^{1}}{d t}\right)^{2}+\left(\frac{d x^{2}}{d t}\right)^{2}+\left(\frac{d x^{3}}{d t}\right)^{2}$. Thus the limiting rate of mass conversion depends upon the velocity since, from Eq. (187)

$$
\dot{\gamma}= \pm a_{0} c \sqrt{1-\frac{v^{2}}{c^{2}}} .
$$

Therefore the maximum rate of mass conversion occurs when $v=0$ and then it may be seen that the limiting rate of mass conversion is given by $\dot{\gamma}_{\max }=a_{0} c$. The limiting rate is specified for a particle which is sitting still, or for $v=0$. However, on the other hand if $v=c$, then there can be no mass conversion. This might be better seen from another view. A system, which we mighi consider as a 
particle, finds itself 1 imited in how fast its mass may change (to be concise we should say "mass density") by the relativistic appearing Eq. (188). On the other hand the speed of light, $c$, represents a limiting velocity and the arguments of special relativity may be reproduced here to show that, for a particle beginning with a velocity less than $c$, no massive particle may be accelerated to the speed of light. Here we find the further conclusion that a particle may not be created and have a velocity equal to $c$ upon creation.

Notice that in the argument above we hedged a little when comparing the argument to an argument based upon Einstein's special theory. The reason for this comes from an important difference between the theories. Einstein's theory is restricted to the four-dimensional world of space-time. Thus it seems reasonable to conclude that if something cannot be accelerated up to the speed of light, then nothing can exist at a velocity greater than the speed of 1 ight. This logic, howevs:r, contains the pitfall of inductive reasoning. The trap appears, not so much in the logic of the Special Theory, as in the limitations imposed by the nature of Einstein's postulates. By this I mean that by adopting Einstein's postulates we are limited to a four-dimensional world and must rule out solutions with velocities greater than the speed of light.

If, on the other hand, we look at the Dynamic Theory, we find that the theory is not restricted to any number of independent variables, or dimensionality. Further, we find that the limiting effect of the third law does not exclude solutions with velocities greater than the speed of light but, in the four-dimensional case, rather rules out solutions with velocities which cross the absolute velocity. This means that within the Dynamic Theory solutions may exist for particles with velocity greater than the speed of light.

The next question might well be, if velocities greater than the speed of light are allowed, how might they be obtained? Obviously, the allowance of these velocities as solutions does not necessarily mean that they can be obtained. Here we find the generality of the Dynamic Theory coming into the picture. It allows a fifth dimension. Equations (187) and (188) were written for a system restricted to remain very near equilibrium so that a flat, or Euclidean, space may be used. Suppose we remove this restriction so that we must consider a curved space. However, suppose that we relax this restriction in a very particular fashion. Let us suppose that the oniy non-constant metric element is $\hat{\mathbf{g}}_{44}$. Then Eq. (187), which is the conditions for the vanishing of the integrating 
factor, may be written,

$$
1-\frac{v^{2}}{c^{2}}-\frac{\hat{g}_{44} \dot{r}^{2}}{a_{0}^{2} c^{2}}=0,
$$

where $\hat{g}_{44}$ may depend upon position and mass density. Then solving for the velocity which drives the integrating factor to zero we find

$$
v_{0}= \pm c \sqrt{1-\frac{\hat{g}_{44} \dot{\gamma}^{2}}{a_{0}^{2} c^{2}}} .
$$

Now suppose we define the speed of light to be the velocity for which tlie integrating factor goes to zero. This definition is consistent with Einstein's definition of light waves traveling along a null trajectory in the fourdimensional space-time manifold. However, in the five-dimensional manifold of the Dynamic Theory this speed of light depends upon the rate of mass conversion and the metric element $\hat{g}_{44}$.

Obviously, if $\hat{g}_{44}$ is negative, then the limiting velocity, $v_{0}$, exceeds the absolute zero velocity $c$, which now may be defined as the limiting velocity in the absence of mass conversion. Thus the attainment of solutions with velocities which are greater than $c$ depends upon the possibility that the metric element $\hat{g}_{44}$ be less than zero.

For cosmological modeling the Dynamic Theory offers the possibility of zero radiation pressure boundary conditions. Further, the theory may offer new insights on black holes. Black holes are considered to have tremendous mass densities and seem to be appropriate for the application of the theory. Further justification of the theory's a'plicability to black holes comes from the recent appearance in the literature of the need to talk of a generalized second law. This generalization appears as a particular combination of the thermodynamic entropy and an area of a black hole.

To quote wald*, "The generalized second law is a truly remarkable law in that it involves three rather distinct fields of physics: thermodynamics,

*Wald, R., 1977, Particle Creation Near Black Holes, Scientific American. 
general relativity, and quantum theory. Is it merely a strange coincidence that this new law appears to be true, or is there some deep, fundamental significance behind it which we do not yet fully appreciate? At the present, I do not feel that we can answer this question." The Dynamic Theory offers an answer for it shows that there is no coincidence in finding a second law involving these three theories to be seen to hold. Indeed that is just what it predicts. The unification of the different physical theories by the adoption of the three generalized laws points out the fundamental significance of a generalized second law.

It thus seems appropriate to offer three further quotes: "The ultimate aim of many theoretical physicists is, first, to define more precisely the range of validity of the currently known laws of physics and, then, to find the new laws of physics that govern the phenomena outside this range. The discovery of such new laws is generally accompanied by a major breakthrough in our understanding of nature." (R. Wald, 1977) "If we wish to find in rational mechanics an a priori foundation for the principles of thermodynamics, we must seek mechanical definitions of temperature and entropy." (J. W. Gibbs) "A Theory is the more impressive the greater the simplicity of its premises are, the more different kinds of things it relates, and the more extended is its area of applicability. Therefore, the deep impression that classical thermodynamics made upon me. It is the only physical theory of universal content conceining which I am convinced that, within the framework of applicability of its basic concepts, it will never be overthrown." (A. Einstein, 1949). So the Dynamic Theory, as a new view of space, time, and matter, appears to me.

\section{REFERENCES}

1. R. Adler, M. Bazin, and M. Schiffer, Introduction to General Relativity, 1965.

2. John W. Dettman, Mathematical Methods in Physics and Engineering, 1969.

3. K. Huang, Statistical Mechanics, 1963.

4. F. London, Quantum Mechanische Deulung der Theorie, Von Weyl, z, Physik. 42, 375-389 (1927).

5. Jerry B. Marion, Classical Electromagnetic Radiation, 1965.

6. Erwin Modelung, Die Mathematischen Hilfsmittel Des Physikers, 1950. 
7. D. ter Haar and H. Wergelande, Elements of Thermodynamics, 1966.

8. H. Wey1, Space-Time-Matter, 1922.

9. Pharis E. Williams, On a Possible Formulation of Particle Dynamics in Terms of Thermodynamic Conceptualizations and the Role of Entropy in it, Thesis, U.S. Nava1 Postgraduate Schoo7, 1976.

10. Pharis E. Williams, The Principles of the Dynamic Theory, Research Report EW-77-4, U.S. Naval Academy, 1977.

11. William B. Zeleny, Introduction to Relativistic Quantum Mechanics, 1975.

12. M. W. Zemansky, Heat and Thermodynamics, 1968. 